\title{
Product of Hessians and Discriminant of Critical Points of Level Function for Hypergeometric Integrals
}

\author{
Kazuhiko Aomoto ${ }^{a, *}$ and Masahiko Ito $^{b}$ \\ ${ }^{a}$ Department of Mathematics, Nagoya University, \\ Furo-cho, Chikusa-ku, Nagoya, 464-8602 Japan \\ ${ }^{b}$ Department of Mathematical Sciences, University of the Ryukyus, \\ Sembaru, Nakagami-gun Nishihara-cho, Okinawa 903-0213, Japan \\ E-mail: aomoto@math.nagoya-u.ac.jp, mito@sci.u-ryukyu.ac.jp
}

We give in two examples (hyperplane arrangement and circle arrangement) a relation between the product of the Hessians of a level function associated with hypergeometric integrals and discriminant attached to them. We also express the product of values of prime factors of integrand at critical points by the use of basic invariants attached to each arrangement. Our main goal is to give a criterion in terms of the product of Hessians for that all critical points are different from each other.

MathemAmplitudes 2019: Intersection Theory \& Feynman Integrals (MA2019)

18-20 December 2019

Padova, Italy

\footnotetext{
${ }^{*}$ Speaker
} 


\section{Introductory explanation (Divergent integral and twisted cycle)}

We begin to explain in an elementary way the notion of "twisted cycle" and how it justifies "finite part of divergent integral" appearing in Mathematical Physics.

The function $x_{+}^{\lambda}$ on $\mathbb{R}$ for $\mathfrak{R e} \lambda>-1$ is an ordinary function but for $\lambda \in \mathbb{C}-\mathbb{Z}, \lambda \leq-1$ is a generalized function defined as follows:

Suppose $f(x)$ is an arbitrary holomorphic function near the origin. Fix a point $a>0$ near the origin. Consider the integral

$$
\left\langle x_{+}^{\lambda}, f\right\rangle=\int_{0}^{a} x^{\lambda} f(x) d x=\lim _{\varepsilon \downarrow 0} \int_{\varepsilon}^{a} x^{\lambda} f(x) d x .
$$

Case 1. Suppose first $-n-1<\mathfrak{R e} \lambda<-n(n=1,2,3, \ldots)$. Then (1.1) is divergent. $f(x)$ has a Taylor expansion at the origin

$$
f(x)=\sum_{m=0}^{n-1} \frac{f^{m}(0)}{m !} x^{m}+x^{n} g(x),
$$

where $g(x)$ is holomorphic on $[0, a]$. The finite part of (1.1) in the sense of J. Hadamard is given as follows :

$$
J(\lambda)=\text { f.p. } \int_{0}^{a} x^{\lambda} f(x) d x=\sum_{m=0}^{n-1} \frac{f^{(m)}(0)}{m !} \frac{a^{\lambda+m+1}}{\lambda+m+1}+\int_{0}^{a} x^{\lambda+n} g(x) d x .
$$

This is the generalized function $x_{+}^{\lambda}$ which has been defined by I. M. Gelfand and G. E. Shilov in the mid 20th century (see [9]), i.e.,

$$
\left\langle x_{+}^{\lambda}, f\right\rangle=\text { f.p. } \int_{0}^{a} x^{\lambda} f(x) d x .
$$

In a neighborhood of the origin we take a path $\sigma_{0}$ starting from and ending in $a$ going around the origin counter-clockwise ("loop based on the point $a$ going around the origin")

$$
\frac{1}{e^{2 \pi i \lambda}-1} \sigma_{0}=[\varepsilon, a]+\frac{1}{e^{2 \pi i \lambda}-1} \delta_{\varepsilon} \quad(\varepsilon>0),
$$

where $\delta_{\varepsilon}$ is a scalar multiple of a loop with base point $\varepsilon$ in a neighborhood of 0 . Then the integral

$$
\frac{1}{e^{2 \pi i \lambda}-1} \int_{\sigma_{0}} x^{\lambda} f(x) d x
$$

equals (1.2). This is called "detoured cycle at the origin". (This idea already can be found in the work of J. Leray in the middle of 20th century).

Case 2. When $\lambda=-n(n=1,2,3, \ldots)$ the finite part is defined as

$$
\text { f.p. } \int_{0}^{a} x^{-n} f(x) d x=\sum_{m=0}^{n-2} \frac{f^{(m)}(0)}{m !} \frac{a^{-n+m+1}}{-n+m+1}+\frac{f^{(n-1)}(0)}{n !} \log a+\int_{0}^{a} g(x) d x .
$$

The generalized function $x_{+}^{-n}$ is then defined by the finite part

$$
\left\langle x_{+}^{-n}, f\right\rangle=\text { f.p. } \int_{0}^{a} x^{-n} f(x) d x .
$$


$J(\lambda)$ has Laurent expansion at $\lambda=-n$

$$
J(\lambda)=\frac{c_{-1}}{\lambda+n}+c_{0}+c_{1}(\lambda+n)+\cdots,
$$

so that the finite part coincides with $c_{0}$, i.e.,

$$
\text { f.p. } \int_{0}^{a} x^{-n} f(x) d x=c_{0}=\lim _{\lambda \rightarrow-n} \frac{d}{d \lambda}(\lambda+n) J(\lambda)=\frac{1}{2 \pi i} \int_{\sigma_{0}} x^{-n}(\log x-\pi i) f(x) d x .
$$

\section{Example 1.1.}

(1) f.p. $\int_{a}^{\infty}(x-a)^{\lambda} d x=0$ for all $\lambda \in \mathbb{R}$.

(2) f.p. $\int_{a}^{b} \frac{f(x)}{x} d x=$ p.v. $\int_{a}^{b} \frac{f(x)}{x} d x=\int_{a}^{b} \frac{f(x)-f(0)}{x} d x+f(0) \log \frac{b}{-a} \quad(a<0<b)$, where the symbol p.v. denotes the principal value.

(3) f.p. $\int_{0}^{\infty} \frac{e^{-x}}{x} d x=\int_{0}^{\infty}\left(\frac{e^{-x}}{x}-\frac{x}{e^{x}-1}\right) d x=\Gamma^{\prime}(1)=-C$,

where $C$ denotes the Euler constant.

\section{Example 1.2. (Beta function) For $\alpha, \beta \notin \mathbb{Z}$}

$$
J(\alpha, \beta)=\text { f.p. } \int_{0}^{1} x^{\alpha}(1-x)^{\beta} d x
$$

is equal to Beta function $B(\alpha, \beta)$. Take $\sigma_{0}, \sigma_{1}$ the loops with the base point $x=\frac{1}{2}$ going around 0,1 in a positive direction respectively. Then

$$
J(\alpha, \beta)=\frac{1}{e^{2 \pi i \alpha}-1} \int_{\sigma_{0}} x^{\alpha}(1-x)^{\beta} d x-\frac{1}{e^{2 \pi i \beta}-1} \int_{\sigma_{1}} x^{\alpha}(1-x)^{\beta} d x .
$$

The monodromy $\mathcal{M}$ associated with the function $\Phi(x)=x^{\alpha}(1-x)^{\beta}$

$$
\sigma_{0} \longrightarrow M\left(\sigma_{0}\right)=e^{2 \pi i \alpha} \in \mathbb{C}^{*}, \quad \sigma_{1} \longrightarrow M\left(\sigma_{1}\right)=e^{2 \pi i \beta} \in \mathbb{C}^{*}
$$

defines the local system $\mathcal{L}$ and its dual $\mathcal{L}^{*}$ on the space $X=\mathbb{C}-\{0,1\}$. The boundary operator $\partial$ acts on the linear space of chains $\mathbf{c}=c_{0} \sigma_{0}+c_{1} \sigma_{1}\left(c_{0}, c_{1} \in \mathbb{C}\right)$ with values in $\mathcal{L}^{*}$ as follows:

$$
\partial\left(c_{0} \sigma_{0}+c_{1} \sigma_{1}\right)=\left(c_{0}\left(e^{2 \pi i \alpha}-1\right)+c_{1}\left(e^{2 \pi i \beta}-1\right)\right)\left\{\frac{1}{2}\right\} .
$$

It is closed (twisted cycle) if and only if

$$
c_{0}\left(e^{2 \pi i \alpha}-1\right)+c_{1}\left(e^{2 \pi i \beta}-1\right)=0 .
$$

Hence the one dimensional homology $H_{1}\left(X, \mathcal{L}^{*}\right)$ is just one dimensional with the basis

$$
\mathbf{c}=\frac{1}{e^{2 \pi i \alpha}-1} \sigma_{0}-\frac{1}{e^{2 \pi i \beta}-1} \sigma_{1} .
$$


We have

$$
J(\alpha, \beta)=\langle\mathbf{c}, d x\rangle .
$$

On the other hand if $\alpha=-n-1(n=0,1,2,3, \ldots)$, then

$$
\begin{aligned}
& J(-n-1, \beta)=\text { f.p. } \int_{0}^{1} x^{-n-1}(1-x)^{\beta} d x \quad(\beta>-1) \\
& =\frac{1}{2 \pi i} \int_{\sigma_{0}} x^{-n-1}(1-x)^{\beta}(\log x-\pi i) d x-\frac{1}{e^{2 \pi i \beta}-1} \int_{\sigma_{1}} x^{-n-1}(1-x)^{\beta} d x .
\end{aligned}
$$

The vector function of two components ${ }^{T}\left((1-x)^{\beta},(1-x)^{\beta} \log x\right)$ (T denotes the transposition) defines the monodromy and the associated local system $\mathcal{L}$ of rank two and its dual $\mathcal{L}^{*}$. The fundamental $2 \times 2$ matrix function $\Phi$ is defined by the lower triangular matrix

$$
\Phi(x)=\left(\begin{array}{cc}
(1-x)^{\beta} & \\
(1-x)^{\beta} \log x & (1-x)^{\beta}
\end{array}\right),
$$

and its monodromy is given by

$$
\mathcal{M} \longrightarrow M\left(\sigma_{0}\right)=\left(\begin{array}{cc}
1 & \\
2 \pi i & 1
\end{array}\right), \quad M\left(\sigma_{1}\right)=\left(\begin{array}{ll}
e^{2 \pi i \alpha} & \\
& e^{2 \pi i \beta}
\end{array}\right) .
$$

The space of chains with coefficients in $\mathcal{L}^{*}$ is the linear space consisting of two components

$$
\mathfrak{c}=\left(c_{11}, c_{12}\right) \sigma_{0}+\left(c_{21}, c_{22}\right) \sigma_{1} \quad\left(c_{j k} \in \mathbb{C}\right) .
$$

The pairing of integral between the chain $\mathfrak{c}$ and two component vector function ${ }^{T}\left(\varphi_{1}(x), \varphi_{2}(x)\right)$ is given by

$$
\left\langle\mathfrak{c},{ }^{T}\left(\varphi_{1}, \varphi_{2}\right)\right\rangle=\int_{\sigma_{0}}\left(c_{11}, c_{12}\right) \Phi(x)^{T}\left(\varphi_{1}, \varphi_{2}\right) d x+\int_{\sigma_{1}}\left(c_{21}, c_{22}\right) \Phi(x)^{T}\left(\varphi_{1}, \varphi_{2}\right) d x .
$$

The boundary operator is given by

$$
\partial(\mathfrak{c})=\left\{\left(c_{11}, c_{12}\right)\left(M\left(\sigma_{0}\right)-I\right)+\left(c_{21}, c_{22}\right)\left(M\left(\sigma_{1}\right)-I\right)\right\}\left\{\frac{1}{2}\right\},
$$

and $\mathfrak{c}$ is closed if and only if

$$
2 \pi i c_{12}+\left(e^{2 \pi i \beta}-1\right) c_{21}=0, \quad\left(e^{2 \pi i \beta}-1\right) c_{22}=0,
$$

i.e.,

$$
c_{22}=0, \quad c_{21}=-\frac{2 \pi i}{e^{2 \pi i \beta}-1} c_{12} .
$$

Hence we have two linearly independent twisted cycles

$$
\mathfrak{c}_{1}=(1,0) \sigma_{0}, \quad \mathfrak{c}_{2}=\left(0, \frac{1}{2 \pi i}\right) \sigma_{0}+\left(-\frac{1}{e^{2 \pi i \beta}-1}, 0\right) \sigma_{1} .
$$

The integral (1.3) is nothing else than the pairing $\left\langle\mathfrak{c}_{2},{ }^{T}\left(x^{-n-1} d x,-\pi i x^{-n-1} d x\right)\right\rangle$, namely

$$
J(-n-1, \beta)=\left\langle\mathfrak{c}_{2},{ }^{T}\left(x^{-n-1} d x,-\pi i x^{-n-1} d x\right)\right\rangle .
$$


Finally we consider the integral

$$
\begin{aligned}
& J(-n-1,-m-1)=\text { f.p. } \int_{0}^{1} x^{-n-1}(1-x)^{-m-1} d x \quad(n, m=0,1,2, \ldots) \\
& =\frac{1}{2 \pi i}\left(\int_{\sigma_{0}} x^{-n-1}(1-x)^{-m-1}(\log x-\pi i) d x-\int_{\sigma_{1}} x^{-n-1}(1-x)^{-m-1}(\log (1-x)-\pi i) d x\right) .
\end{aligned}
$$

The vector function of three components $1, \log x, \log (1-x)$ defines the $3 \times 3$ fundamental function matrix

$$
\Phi(x)=\left(\begin{array}{ccc}
1 & & \\
\log x & 1 & \\
\log (1-x) & 0 & 1
\end{array}\right)
$$

and the corresponding monodromy is given by

$$
M\left(\sigma_{0}\right)=\left(\begin{array}{ccc}
1 & & \\
2 \pi i & 1 & \\
0 & 0 & 1
\end{array}\right), \quad M\left(\sigma_{1}\right)=\left(\begin{array}{ccc}
1 & & \\
0 & 1 & \\
2 \pi i & 0 & 1
\end{array}\right) .
$$

The local system has rank three and the twisted cycle is represented by

$$
\mathfrak{c}=\left(c_{11}, c_{12}, c_{13}\right) \sigma_{0}+\left(c_{21}, c_{22}, c_{23}\right) \sigma_{1}
$$

such that

$$
\left(c_{11}, c_{12}, c_{13}\right) M\left(\sigma_{0}\right)+\left(c_{21}, c_{22}, c_{23}\right) M\left(\sigma_{1}\right)=0
$$

i.e.,

$$
c_{12}+c_{23}=0 .
$$

The above integral (1.5) gives the pairing between the cycle

$$
\mathfrak{c}_{3}=\left(-\frac{1}{2}, \frac{1}{2 \pi i}, 0\right) \sigma_{0}+\left(\frac{1}{2}, 0,-\frac{1}{2 \pi i}\right) \sigma_{1}
$$

and the three component vector one-form ${ }^{T}\left(x^{-n-1}(1-x)^{-m-1} d x, 0,0\right)$ :

$$
J(-n-1,-m-1)=\left\langle\mathfrak{c}_{3},{ }^{T}\left(x^{-n-1}(1-x)^{-m-1} d x, 0,0\right)\right\rangle .
$$

Let $\mathcal{L}_{l f}$ be the same local system on $X$ which is locally finite at the singularity $0,1, \infty$ and $\mathcal{L}_{l f}^{*}$ be its dual. There is a canonical morphism "reg" often called the "regularization" or "renormalization"

$$
\begin{aligned}
& \text { reg }: H_{1}\left(X, \mathcal{L}_{l f}^{*}\right) \rightarrow H_{1}\left(X, \mathcal{L}^{*}\right) \\
& \uparrow \\
& H_{1}\left(X, \mathcal{L}_{l f}\right)
\end{aligned}
$$

such that $\operatorname{reg}[0,1]=\mathfrak{c}$ in $(1.3)$ and $\operatorname{reg}[0,1]=\mathfrak{c}_{2}$ in $(1.4)$ or $\mathfrak{c}_{3}$ in $(1.5)$. 


\section{Asymptotics for large exponents and critical points}

Let us begin with a simplest example.

Example 2.1. For different $a_{j} \in \mathbb{C}(1 \leq j \leq m)$ and $\lambda=\sum_{j=1}^{m} \lambda_{j} \varepsilon_{j} \in \mathbb{R}^{m}\left(\left\{\varepsilon_{j}\right\}_{1 \leq j \leq m}\right.$ means the standard basis of $\mathbb{R}^{m}$ ) we take

$$
\Phi(w)=\prod_{j=1}^{m}\left(w-a_{j}\right)^{\lambda_{j}}
$$

and the integral over a twisted cycle $\mathfrak{z}$ in the space $X=\mathbb{C}-\bigcup_{j=1}^{m}\left\{a_{j}\right\}$

$$
J_{\lambda}(\varphi)=\int_{\mathfrak{z}} \Phi(w) \varphi(w) d w
$$

where $\varphi(w) d w$ is a rational differential one-form which is holomorphic on $X$. Denote by $H_{\nabla}^{1}(X, \Omega)$ the one dimensional twisted de Rham cohomology with respect to the covariant derivation

$$
\nabla: \psi \longrightarrow \nabla \psi=d \psi+\sum_{j=1}^{m} \lambda_{j} d \log \left(w-a_{j}\right) \wedge \psi
$$

for $\psi \in \Omega^{0}$ (scalar valued) (see [3]). Denote the logarithmic one forms $\varphi_{j}(w) d w=d \log \left(w-a_{j}\right)$ $(1 \leq j \leq m)$. One can take $\varphi_{j}(w) d w(1 \leq j \leq m-1)$ as the representative of the basis of $H_{\nabla}^{1}\left(X, \Omega^{*}\right)$ (Orlik-Solomon basis [12]). The shift operator $T_{\varepsilon_{j}}$ associated with the shift : $\lambda \rightarrow \lambda+\varepsilon_{j}$ acts on $H_{\nabla}^{1}\left(X, \Omega^{*}\right)$ :

$$
T_{\varepsilon_{j}}\left(\varphi_{k} d w\right) \sim \sum_{l=1}^{m-1} \varphi_{l} d w a_{j ; l k}(\lambda) \quad \text { (homologically). }
$$

The $(m-1) \times(m-1)$ matrices $A_{j}(\lambda)=\left(a_{j ; l k}(\lambda)\right)_{1 \leq k, l \leq m-1}$ are rational functions of $\lambda$ which have the asymptotic expansions

$$
A_{j}(\lambda)=A_{j}^{(0)}+O\left(\frac{1}{N}\right) \quad\left(\lambda=N v+\lambda^{\prime}\right)
$$

where $A_{j}^{(0)}$ commute with each other under the generic condition $(C)$ :

$$
(C): a_{j} \neq a_{k} \quad(j \neq k) \text {. }
$$

Put $\lambda=N v+\lambda^{\prime}$ with $v=\sum_{j=1}^{m} v_{j} \varepsilon_{j} \in \mathbb{Z}^{m}-\{0\}$, where $\lambda^{\prime}=\sum_{j=1}^{m} \lambda_{j}^{\prime} \varepsilon_{j}$ is fixed. We are interested in the asymptotic behavior of $J_{\lambda}(\varphi)$ when $N \in \mathbb{Z}_{>0}$ tends to the infinity in the direction $v$. Take

$$
F=\sum_{j=1}^{m} v_{j} \log \left(w-a_{j}\right)
$$

For the real valued level function $\mathfrak{R e}(F)$ the associated critical points $\zeta_{j} \in \mathbb{C}(1 \leq j \leq m-1)$ satisfy the equality

$$
\frac{d F}{d w}=\sum_{j=1}^{m} \frac{v_{j}}{w-a_{j}}=0 .
$$


Generally there are $m-1$ different critical points $\zeta_{j}$. To each point $\zeta_{j}$ there exists the one dimensional stable cycle $\mathfrak{z}_{j}$ which is Lagrangian. This is locally described at $\zeta_{j}$ by

$$
\mathfrak{I m} F(w)=\mathfrak{I m} F\left(\zeta_{j}\right)
$$

There also exists the one dimensional unstable cycle $\mathfrak{z}_{j}^{-}$at $\zeta_{j}$. Each of the systems $\mathfrak{z}_{j}(1 \leq j \leq m-1)$ and $\mathfrak{z}_{j}^{-}(1 \leq j \leq m-1)$ makes a basis of $H_{1}\left(X, \mathcal{L}^{*}\right)$. They give the asymtotics of integral in the direction $v$ and $-v$ respectively.

Now for simplicity we consider the case $m=3$ where $v=\varepsilon_{1}+\varepsilon_{2}+\varepsilon_{3}$ i.e., $v_{1}=v_{2}=v_{3}=1$. Then we have

$$
A_{1}(\lambda)=\left(\begin{array}{cc}
\frac{\lambda_{1}}{1+\lambda_{\infty}}\left(a_{3}-a_{1}\right) & \frac{\lambda_{1}}{1+\lambda_{\infty}}\left(a_{3}-a_{1}\right) \\
\frac{\lambda_{2}}{1+\lambda_{\infty}}\left(a_{3}-a_{2}\right) & \frac{\lambda_{2}}{1+\lambda_{\infty}}\left(a_{3}-a_{2}\right)+\left(a_{2}-a_{1}\right)
\end{array}\right)
$$

and

$$
A_{1}^{(0)}=\left(\begin{array}{cc}
\frac{a_{3}-a_{1}}{3} & \frac{a_{3}-a_{1}}{3} \\
\frac{a_{3}-a_{2}}{3} & \frac{a_{3}+2 a_{2}-3 a_{1}}{3}
\end{array}\right),
$$

where $\lambda_{\infty}=\lambda_{1}+\lambda_{2}+\lambda_{3}$. The multiplication by the variable $w: T_{w}=A_{1}+a_{1} I$ corresponds to the matrix

$$
A_{w}^{(0)}=A_{1}^{(0)}+a_{1} I=\left(\begin{array}{cc}
\frac{a_{3}+2 a_{1}}{3} & \frac{a_{3}-a_{1}}{3} \\
\frac{a_{3}-a_{2}}{3} & \frac{a_{3}+2 a_{2}}{3}
\end{array}\right) .
$$

This has the eigenvalues $\zeta_{1}, \zeta_{2}$. One can easily show that $\zeta_{1}, \zeta_{2}$ both lie in the inside of the triangle with vertices $a_{1}, a_{2}, a_{3}$. The discriminant of (2.2) is given by the determinant of Hankel matrix $\mathcal{H}_{1}$ of $A_{w}^{(0)}$ (see [8]):

$$
\mathcal{H}_{1}=\left(\begin{array}{cc}
\operatorname{Tr}(I) & \operatorname{Tr}\left(A_{w}^{(0)}\right) \\
\operatorname{Tr}\left(A_{w}^{(0)}\right) & \operatorname{Tr}\left(\left\{A_{w}^{(0)}\right\}^{2}\right)
\end{array}\right)
$$

and

$$
\operatorname{det} \mathcal{H}_{1}=\left(\zeta_{1}-\zeta_{2}\right)^{2}=a_{1}^{2}+a_{2}^{2}+a_{3}^{2}-a_{1} a_{2}-a_{1} a_{3}-a_{2} a_{3} .
$$

Under the condition $(C)$ one can obtain the product formula

$$
\left[\frac{d^{2} F}{d w^{2}}\right]_{w=\zeta_{1}} \cdot\left[\frac{d^{2} F}{d w^{2}}\right]_{w=\zeta_{2}}=\frac{\left(\zeta_{1}-\zeta_{2}\right)^{2}}{3\left(a_{1}-a_{2}\right)^{2}\left(a_{1}-a_{3}\right)^{2}\left(a_{2}-a_{3}\right)^{2}} .
$$

The two critical points meet each other if and only if $\prod_{j=1}^{2}\left[\frac{d^{2} F}{d w^{2}}\right]_{w=\zeta_{j}}$ vanishes. This occurs if and only if $a_{1}, a_{2}, a_{3}$ are the vertices of a regular triangle and $\zeta_{1}=\zeta_{2}$ is the center of gravity.

\section{Problems, Methods and Main Results}

For large exponents the behavior of critical points of a level function gives an influence for asymptotics of corresponding hypergeometric integral. In this article we want to show in an explicit way how the product of Hessians of the level function at all critical points is involved in the behavior of its critical points. 
Let $f_{j}=f_{j}(x)(1 \leq j \leq m)$ be real polynomials in $x=\left(x_{1}, \ldots, x_{n}\right)$ in the affine space $\mathbb{C}^{n}$. Let $X$ be the affine manifold which is the complement of the union of the hypersurfaces $S_{j}: f_{j}=0$, i.e.,

$$
X=\mathbb{C}^{n}-\bigcup_{j=1}^{m} S_{j}
$$

The hypergeometric integral with respect to the multiplicative function

$$
\Phi(x)=\prod_{j=1}^{m} f_{j}^{\lambda_{j}}
$$

with exponents $\lambda=\sum_{j=1}^{m} \lambda_{j} \varepsilon_{j} \in \mathbb{R}^{m}\left(\varepsilon_{j}\right.$ denotes the standard basis of $\left.\mathbb{R}^{m}\right)$ is defined by

$$
J(\varphi)=\int \Phi(x) \varphi(x) d x_{1} \wedge \cdots \wedge d x_{n} \quad\left(\varphi \in \Omega^{*}\right) .
$$

$H_{\nabla}^{n}\left(X, \Omega^{\prime}\right)$ denotes the $n$ dimensional twisted cohomology on $X$ with respect to the covariant differentiation:

$$
\nabla \varphi=d \varphi+\sum_{j=1}^{m} \lambda_{j} d \log f_{j} \wedge \varphi
$$

Its dual is isomorphic to the $n$ dimensional twisted homology $H_{n}\left(X, \mathcal{L}^{*}\right)$ where $\mathcal{L}^{*}$ denotes the dual local system associated with the function $\Phi$. The perfect pairing between them can be described by the above integral.

Let $\lambda^{\prime} \in \mathbb{R}^{m}$ and $\boldsymbol{v}=\sum_{j=1}^{m} v_{j} \varepsilon_{j} \in \mathbb{Z}^{m}-\{0\}$ be fixed. Put $\lambda=N \boldsymbol{v}+\lambda^{\prime}$ for a positive integer $N$. Denote $|v|=\sum_{j=1}^{m}\left|v_{j}\right|$. We consider the asymptotic behavior of the integral $J(\varphi)$ for a large $N$. One can define the real valued level function $\mathfrak{R e} F$ from the logarithm

$$
F(x)=\sum_{j=1}^{m} v_{j} \log f_{j} .
$$

The singularity of the gradient flow of $\mathbf{v}=\operatorname{grad} \mathfrak{R e} F$ in $X$ coincides with its critical points $\mathbf{c}_{k}$ of $F$ satisfying the equation:

$$
0=d F=\sum_{j=1}^{m} v_{j} d \log f_{j} .
$$

A system of linearly independent representatives of $H_{n}\left(X, \mathcal{L}^{*}\right)$ is obtained by stable cycles $\mathfrak{z} k$ $(1 \leq k \leq \kappa)$ which are Lagrangian.

Suppose the critical point $\mathbf{c}_{k}$ is non-degenerate. Then there exists a system of local coordinates $\xi=\left(\xi_{1}, \ldots, \xi_{n}\right)$ such that the origin corresponds to $\mathbf{c}_{k}$ and $\xi$ is real on the stable cycle $\mathfrak{z}_{k}$ (see [3, Theorem 4.6]). The Hessian of $F$ at $\mathbf{c}_{k}$ is defined by

$$
[\operatorname{Hess}(F)]_{\mathbf{c}_{k}}=\left[\frac{\operatorname{det}\left(\frac{\partial^{2} F}{\partial \xi_{j} \partial \xi_{k}}\right)_{1 \leq j, k \leq n}}{\left\{\operatorname{det}\left(\frac{\partial x_{j}}{\partial \xi_{k}}\right)_{1 \leq j, k \leq n}\right\}^{2}}\right]_{\xi=0} .
$$

If $\varphi$ does not depend on $\lambda$, we have the following for large $N$ by saddle point method:

$$
\left\langle\varphi, \mathfrak{z}_{k}\right\rangle=\int_{\mathfrak{z} k} \Phi \varphi \approx \Phi\left(\mathbf{c}_{k}\right) \varphi\left(\mathbf{c}_{k}\right) \frac{(2 \pi)^{\frac{n}{2}}}{\sqrt{N^{n}(-1)^{n}[\operatorname{Hess}(F)]_{\mathbf{c}_{k}}}} .
$$


Under a suitable "non-resonance" condition, $\kappa$ equals the dimension of the twisted cohomology $H_{\nabla}^{n}\left(X, \Omega^{*}\right)$.

Denote by $\varphi_{j} d x_{1} \wedge \cdots \wedge d x_{n}(1 \leq j \leq \kappa)$ the representative of a basis of $H_{\nabla}^{n}(X, \Omega)$. The Wronskian $W$ is defined by the determinant $\operatorname{det} Y$ of the fundamental $\kappa \times \kappa$ matrix $Y=\left(\left\langle\varphi_{j}, \mathfrak{z}_{k}\right\rangle_{j, k}\right)$.

We have the following asymptotic expansion for large $N$ :

$$
W \approx \prod_{k=1}^{\kappa}\left\{\exp \left[N F\left(\mathbf{c}_{k}\right)\right] \prod_{j=1}^{m} f_{j}^{\lambda_{j}^{\prime}}\left(\mathbf{c}_{k}\right) \varphi_{j}\left(\mathbf{c}_{k}\right)\right\} \cdot N^{-\frac{n \kappa}{2}}(2 \pi)^{\frac{n \kappa}{2}}\left(w_{0}+\frac{w_{1}}{N}+\frac{w_{2}}{N^{2}}+\cdots\right),
$$

where

$$
w_{0}=\prod_{k=1}^{\kappa} \frac{1}{\sqrt{(-1)^{n}[\operatorname{Hess}(F)]_{\mathbf{c}_{k}}}} .
$$

We can now pose several questions as follows.

Question 1. Evaluate $\mathcal{N}\left(f_{j}\right):=\prod_{k=1}^{K} f_{j}\left(\mathbf{c}_{k}\right)$.

Question 2. Evaluate $\mathcal{N}(\operatorname{Hess}(F)):=\prod_{k=1}^{K}[\operatorname{Hess}(F)]_{\mathbf{c}_{k}}$.

Question 3. When $\mathcal{N}(\operatorname{Hess}(F))$ vanishes?

Question 4. Under which condition all the critical points are real?

There is an interesting analogy between $f_{j}$ and the quantity $[\operatorname{Hess}(F)]_{\mathbf{c}_{k}}$ on the one hand and the notion of "norm", "unit" and "differente" in algebraic number theory on the other (see $[1,2,11,14]$ ). In the moduli space for the polynomials $\left\{f_{k}\right\}_{1 \leq k \leq m}, f_{j}^{-1}$ is also regular in $X$ because $f_{j}\left(\mathbf{c}_{k}\right)$ never vanishes. In this sense $f_{j}$ is regarded as "unit". However $\operatorname{Hess}(F)$ may vanish sometimes at $\mathbf{c}_{k}$. In the sequel for a rational function $\varphi$ on $X$ the product $\prod_{1 \leq j \leq k}[\varphi]_{\mathbf{c}_{j}}$ will be called "norm" of $\varphi$ and be denoted by $\mathcal{N}(\varphi) . \varphi$ is called a unit if and only if $\mathcal{N}(\varphi)$ never vanishes anywhere. One may conjecture the following:

Ansatz. The product

$$
\prod_{k=1}^{\kappa}[\operatorname{Hess}(F)]_{\mathbf{c}_{k}}=\mathcal{N}(\operatorname{Hess}(F))
$$

is expressed as

$$
\mathcal{N}(\operatorname{Hess}(F))=\mathcal{N}(\text { unit }) \cdot \text { Discr, }
$$

where Discr means the "discriminant" associated with the set of critical points $\mathbf{c}_{j}(1 \leq j \leq \kappa)$ and $\mathcal{N}$ (unit) never vanishes. It vanishes if and only if a pair of the critical points $\mathbf{c}_{k}$ coincides with each other.

Remark. A general definition of "discriminant" for a finite dimensional algebra has been introduced by S. S. Abhyanker (see [1, 2]).

In this article we want to give two examples showing that $\mathcal{N}(\operatorname{Hess}(F))$ has an intimate connection with the "discriminant". It may play the similar role of "discriminants" as in algebraic number theory (see $[11,14]$ ). First we find a rational curve $x=\omega(\tau)(\tau \in \mathbb{C})$ in $X$ such that this curve passes through all the critical points $\mathbf{c}_{j}$ such that $\mathbf{c}_{j}=\omega\left(\tau_{j}\right)(1 \leq j \leq \kappa)$. Then we show that 
"Discr" can be expressed by using the product $\prod_{1 \leq j<k \leq k}\left(\tau_{j}-\tau_{k}\right)^{2}$. See Theorems 4.4, 4.7 (in the case of hyperplane arrangement) and Propositions 5.7, 5.11, Theorems 5.19, 6.5, 7.14, 7.15 (in the case of circle arrangement). It is expected that these Theorems and Propositions could be extended to more general hypergeometric integrals.

\section{Hyperplane Arrangements}

Let $f_{j}(1 \leq j \leq n+2)$ be the following linear functions with real coefficients:

$$
f_{j}:=x_{j}(1 \leq j \leq n), \quad f_{n+1}:=1-\sum_{k=1}^{n} x_{k}, \quad f_{n+2}:=1-\sum_{k=1}^{n} u_{k} x_{k}
$$

for the parameter $u=\left(u_{1}, \ldots, u_{n}\right) \in \mathbb{R}^{n}$ under the condition $\left(C_{1}\right)$ :

$$
\left(C_{1}\right): u_{j} \neq u_{k}(j \neq k), \quad u_{j} \notin\{0,1\} \quad(1 \leq j \leq n) .
$$

This gives the moduli space of the arrangement of $n+2$ real hyperplanes in general position.

Under $\left(C_{1}\right)$ it is known that for generic $\lambda$ such that all $\lambda_{j}>0$ one has $\kappa=n+1$, and that one can choose as the representative of a basis of $H_{n}\left(X, \mathcal{L}^{*}\right)$ the regularization of the compact chambers of the associated real hyperplane arrangements corresponding to the components of the complement of $\bigcup_{j=1}^{m} S_{j}$ (refer to $\left.[3,12,15]\right)$ :

$$
\mathfrak{R e} X=\mathbb{R}^{n} \cap X .
$$

Suppose now that all $v_{j}(1 \leq j \leq n+2)$ and $v_{\infty}=\sum_{k=1}^{n+2} v_{k}$ are different from 0 :

$$
v_{\infty} \prod_{j=1}^{n+2} v_{j} \neq 0
$$

(3.1) is equivalent to the system of equations

$$
0=G_{j}:=\frac{v_{j}}{x_{j}}-\frac{v_{n+1}}{f_{n+1}}-\frac{v_{n+2} u_{j}}{f_{n+2}} \quad(1 \leq j \leq n)
$$

This system generally gives $n+1$ solutions, namely $n+1$ critical points (real or complex) of $\mathfrak{R e} F$ (and so of $F$ ) in $X$. We denote them by $\mathbf{c}_{j}(1 \leq j \leq n+1)$. Denote

$$
\tilde{G}_{j}=\frac{G_{j}}{v_{n+1} f_{n+2}+v_{n+2} u_{j} f_{n+1}} \quad(1 \leq j \leq n) .
$$

It follows from (4.1)

$$
\begin{aligned}
x_{j} & =v_{j} \frac{f_{n+1} f_{n+2}}{v_{n+1} f_{n+2}+v_{n+2} u_{j} f_{n+1}}-f_{j} f_{n+1} f_{n+2} \tilde{G}_{j} \quad(1 \leq j \leq n), \\
1-f_{n+1} & =\sum_{k=1}^{n} v_{k} \frac{f_{n+1} f_{n+2}}{v_{n+1} f_{n+2}+v_{n+2} u_{j} f_{n+1}}-\sum_{k=1}^{n} f_{n+1} f_{n+2} f_{j} \tilde{G}_{k}, \\
1-f_{n+2} & =\sum_{k=1}^{n} v_{k} u_{k} \frac{f_{n+1} f_{n+2}}{v_{n+1} f_{n+2}+v_{n+2} u_{k} f_{n+1}}-\sum_{k=1}^{n} f_{n+1} f_{n+2} u_{k} f_{k} \tilde{G}_{k} .
\end{aligned}
$$


For two rational functions $\varphi_{1}, \varphi_{2}$ on $X$ we call "congruent" and denote by $\varphi_{1} \equiv \varphi_{2}$ if they have equal values at all $\mathbf{c}_{j}$. Hence

$$
\begin{aligned}
x_{j} & \equiv v_{j} \frac{f_{n+1} f_{n+2}}{v_{n+1} f_{n+2}+v_{n+2} u_{j} f_{n+1}} \quad(1 \leq j \leq n), \\
1-f_{n+1} & \equiv \sum_{k=1}^{n} v_{k} \frac{f_{n+1} f_{n+2}}{v_{n+1} f_{n+2}+v_{n+2} u_{j} f_{n+1}}, \\
1-f_{n+2} & \equiv \sum_{k=1}^{n} v_{k} u_{k} \frac{f_{n+1} f_{n+2}}{v_{n+1} f_{n+2}+v_{n+2} u_{k} f_{n+1}} .
\end{aligned}
$$

Introduce the new parameter $t=f_{n+2} / f_{n+1}$ as basic parameter. We want to find out the system of rational functions $\omega(t)=\left(\omega_{1}\left(t_{1}\right), \ldots, \omega_{n}(t)\right)$ such that

$$
x_{j} \equiv \omega_{j}(t)
$$

i.e., $x=\omega(t)$ represents a rational curve in $X$ interpolating the set of critical points $\left\{\mathbf{c}_{j} \mid 1 \leq j \leq\right.$ $n+1\}$.

Lemma 4.1. At all critical points $\mathbf{c}_{j}$, $t$ satisfies the algebraic equation of $(n+1)$ th degree:

$$
\psi(t):=1-\frac{1}{t}-\sum_{j=1}^{n} \frac{v_{j}\left(1-u_{j}\right)}{v_{n+1} t+v_{n+2} u_{j}}=0 .
$$

In particular if $\frac{v_{j}}{v_{n+1}}\left(1-u_{j}\right)$ are all positive then all the roots are real and different. Hence $\mathbf{c}_{j}$ are all real and different.

Proof. In fact from (2.1), (2.2) we have

$$
\begin{gathered}
\frac{1}{f_{n+1}} \equiv 1+\sum_{j=1}^{n} \frac{v_{j} t}{v_{n+1} t+v_{n+2} u_{j}}, \\
\frac{1}{f_{n+2}} \equiv 1+\sum_{j=1}^{n} \frac{v_{j} u_{j}}{v_{n+1} t+v_{n+2} u_{j}} .
\end{gathered}
$$

These two equations imply Lemma 4.1.

Furthermore

Lemma 4.2. The following identities hold:

$$
\begin{aligned}
f_{j}=x_{j} & \equiv \frac{v_{j}\left(v_{n+1} t+v_{n+2}\right)}{v_{\infty}\left(v_{n+1} t+v_{n+2} u_{j}\right)} \quad(1 \leq j \leq n), \\
f_{n+1} & \equiv \frac{v_{n+1} t+v_{n+2}}{v_{\infty} t} \\
f_{n+2} & \equiv \frac{v_{n+1} t+v_{n+2}}{v_{\infty}} .
\end{aligned}
$$


Proof. (4.7) and (4.8) show that

$$
\frac{v_{n+2}}{f_{n+2}}-\sum_{k=1}^{n} v_{k} \equiv v_{n+2}-\sum_{k=1}^{n} \frac{v_{n+1} v_{k} t}{v_{n+1} t+v_{n+2} u_{k}} \equiv v_{n+2}-v_{n+1}\left(\frac{1}{f_{n+1}}-1\right) \equiv v_{n+1}+v_{n+2}-\frac{v+1}{f_{n+1}}
$$

i.e.,

$$
\frac{v_{n+1}}{f_{n+1}}+\frac{v_{n+2}}{f_{n+2}} \equiv v_{\infty}
$$

Hence we have

$$
\frac{1}{f_{n+1}}\left(v_{n+1}+\frac{v_{n+2}}{t}\right)=v_{\infty}
$$

which implies (4.10) and so (4.11). On the other hand (4.5) shows

$$
x_{j} \equiv \frac{v_{j} f_{n+2}}{v_{n+1} t+v_{n+2} u_{j}},
$$

which is nothing else than (4.9) because of (4.10).

Put

$$
\omega_{j}(t):=\frac{v_{j}\left(v_{n+1} t+v_{n+2}\right)}{v_{\infty}\left(v_{n+1} t+v_{n+2} u_{j}\right)} \quad(1 \leq j \leq n)
$$

then the rational curve $x_{j}=\omega_{j}(t)(t \in \mathbb{C})$ interpolates the set of critical points $\mathbf{c}_{j}(1 \leq j \leq n+1)$ in $X$ such that $t\left(\mathbf{c}_{j}\right)$ correspond to the roots of the equation $\psi(t)=0$. Denote by $\bar{\psi}(t)$ the monic polynomial of $(n+1)$ th degree which $t$ has the same roots as (4.6)

$$
v_{n+1}^{n} \bar{\psi}(t)=t \prod_{j=1}^{n}\left(v_{n+1} t+v_{n+2} u_{j}\right) \psi(t)=v_{n+1}^{n}\left(t-\zeta_{1}\right) \cdots\left(t-\zeta_{n+1}\right),
$$

where $\zeta_{j}$ denote the zeros of $\bar{\psi}(t) . \bar{\psi}(t)$ is the characteristic polynomial attached to $t$ such that $\zeta_{j}=t\left(\mathbf{c}_{j}\right)$

Definition 4.3. For a rational function $\varphi$ on $X$ we define the "norm" associated with the system of critical points $\mathbf{c}_{j}(1 \leq j \leq n+1)$ as follows:

$$
\mathcal{N}(\varphi):=\prod_{j=1}^{n+1}[\varphi]_{\mathbf{c}_{j}}
$$

We say that $\varphi$ is the "unit" if $\mathcal{N}(\varphi) \neq 0$.

The first main theorem can be stated as follows: 
Theorem 4.4. The following formulae hold:

(1) $\mathcal{N}(t)=(-1)^{n} \frac{v_{n+2}^{n} \prod_{k=1}^{n} u_{k}}{v_{n+1}^{n}}$

(2) $\mathcal{N}\left(v_{n+1} t+v_{n+2} u_{j}\right)=-v_{n+2}^{n} v_{j} u_{j}\left(1-u_{j}\right) \prod_{\substack{1 \leq k \leq n \\ k \neq j}}\left(u_{j}-u_{k}\right) \quad(1 \leq j \leq n)$,

(3) $\mathcal{N}\left(v_{n+1} t+v_{n+2}\right)=v_{\infty} v_{n+2}^{n} \prod_{k=1}^{n}\left(1-u_{k}\right)$,

(4) $\mathcal{N}\left(f_{j}\right)=\frac{v_{j}^{n}}{v_{\infty}^{n} u_{j}} \frac{\prod_{k \neq j}\left(1-u_{k}\right)}{\prod_{k \neq j}\left(u_{j}-u_{k}\right)} \quad(1 \leq j \leq n)$,

(5) $\mathcal{N}\left(f_{n+1}\right)=(-1)^{n} \frac{v_{n+1}^{n}}{v_{\infty}^{n}} \prod_{k=1}^{n} \frac{1-u_{k}}{u_{k}}$,

(6) $\mathcal{N}\left(f_{n+2}\right)=\frac{v_{n+2}^{n}}{v_{\infty}^{n}} \prod_{k=1}^{n}\left(1-u_{k}\right)$.

In particular $f_{j}(1 \leq j \leq n+2)$ are all units in the above sense.

Proof. By definition $\bar{\psi}(t)$ has the following expression:

$$
v_{n+1}^{n} \bar{\psi}(t)=(t-1) \prod_{k=1}^{n}\left(v_{n+1} t+v_{n+2} u_{k}\right)-\sum_{l=1}^{n} v_{l}\left(1-u_{l}\right) t \prod_{\substack{1 \leq k \leq n \\ k \neq j}}\left(v_{n+1} t+v_{n+2} u_{k}\right) .
$$

Hence we have

$$
\mathcal{N}(t)=(-1)^{n+1} \bar{\psi}(0)=(-1)^{n} \prod_{k=1}^{n} \frac{v_{n+2} u_{k}}{v_{n+1}} .
$$

This proves (1). In the same way we have

$$
\mathcal{N}\left(v_{n+1} t+v_{n+2} u_{j}\right)=(-1)^{n+1} v_{n+1}^{n+1} \bar{\psi}\left(-\frac{v_{n+2} u_{j}}{v_{n+1}}\right)=v_{n+2}^{n} v_{j}\left(1-u_{j}\right) u_{j} \prod_{\substack{1 \leq k \leq n \\ k \neq j}}\left(u_{j}-u_{k}\right),
$$

which proves (2), and have

$$
\begin{aligned}
v_{n+1}^{n} \bar{\psi}\left(-\frac{v_{n+2}}{v_{n+1}}\right) & =-\left(\frac{v_{n+2}}{v_{n+1}}+1\right) v_{n+2}^{n} \prod_{k=1}^{n}\left(-1+u_{k}\right)-\sum_{l=1}^{n} v_{l}\left(1-u_{l}\right)\left(-\frac{v_{n+2}^{n}}{v_{n+1}}\right) \prod_{\substack{1 \leq k \leq n \\
k \neq j}}\left(-1+u_{k}\right) \\
& =-\frac{v_{n+2}^{n} v_{\infty}}{v_{n+1}} \prod_{l=1}^{n}\left(-1+u_{k}\right),
\end{aligned}
$$

and

$$
\mathcal{N}\left(v_{n+1} t+v_{n+2}\right)=(-1)^{n+1} v_{n+1}^{n+1} \bar{\psi}\left(-\frac{v_{n+2}}{v_{n+1}}\right) .
$$

This proves (3). (4) follows from (4.9) in view of (2) and (3). (5) follows from (4.10) and (3). (6) follows from (4.11) and (3). 
Now we want to evaluate $\mathcal{N}(\operatorname{Hess}(F))$ in terms of discriminant. One has the obvious identity

$$
\bar{\psi}^{\prime}\left(\zeta_{j}\right)=\left[t \prod_{j=1}^{n}\left(t+\frac{v_{n+2}}{v_{n+1}} u_{j}\right)\right]_{\zeta_{j}}\left[\psi^{\prime}(t)\right]_{\zeta_{j}} .
$$

Put further

$$
\begin{aligned}
G_{1}^{*} & :=-f_{n+1} \sum_{k=1}^{n}\left(1-u_{k}\right) f_{k} G_{k}, \\
G_{2}^{*} & :=f_{n+1} f_{n+2} \sum_{k=1}^{n} f_{k} G_{k}, \\
G_{j}^{*} & :=-f_{j} f_{n+1} f_{n+2} G_{j} \quad(3 \leq j \leq n),
\end{aligned}
$$

then (4.2), (4.3) and (4.4) are expressed in terms of the coordinates $x_{1}^{*}=t_{1}, x_{2}^{*}=f_{n+1}, x_{j}^{*}=x_{j}$ $(3 \leq j \leq n)$ as follows:

$$
\begin{aligned}
G_{1}^{*} & =-f_{n+1} x_{1}^{*} \psi\left(x_{1}^{*}\right), \\
G_{2}^{*} & =1-x_{2}^{*}\left(1+\sum_{k=1}^{n} \frac{v_{k} x_{1}^{*}}{v_{n+1} x_{1}^{*}+v_{n+2} u_{k}}\right), \\
G_{j}^{*} & =x_{j}^{*}-\frac{v_{j}\left(v_{n+1} x_{1}^{*}+v_{n+2}\right)}{v_{\infty}\left(v_{n+1} x_{1}^{*}+v_{n+2} u_{j}\right)} \quad(3 \leq j \leq n) .
\end{aligned}
$$

Under the condition $\left(C_{1}\right)$ the system of equations (4.1) in $X$ is equivalent to the following:

$$
G_{j}^{*}=0 \quad(1 \leq j \leq n)
$$

As a consequence of (4.12) - (4.14)

$$
\begin{aligned}
& \frac{\partial\left(x_{1}^{*}, G_{2}^{*}, \ldots, G_{n}^{*}\right)}{\partial\left(x_{1}^{*}, x_{2}^{*}, \ldots, x_{n}^{*}\right)} \equiv-\frac{1}{x_{2}^{*}}=-\frac{1}{f_{n+1}} \\
& \frac{\partial\left(x_{1}^{*}, x_{2}^{*}, \ldots, x_{n}^{*}\right)}{\partial\left(x_{1}, x_{2}, \ldots, x_{n}\right)}=\frac{u_{1}-u_{2}}{x_{2}^{*}}=\frac{u_{1}-u_{2}}{f_{n+1}} .
\end{aligned}
$$

By definition we have

$$
\begin{aligned}
d \tilde{G}_{1} \wedge \cdots \wedge d \tilde{G}_{n} & \equiv \frac{1}{\left(v_{n+1} f_{n+2}+v_{n+2} u_{j} f_{n+1}\right)^{n}} d G_{1} \wedge \cdots \wedge d G_{n} \\
& =\frac{1}{f_{n+1}^{n}\left(v_{n+1} t+v_{n+2} u_{j}\right)^{n}} d G_{1} \wedge \cdots \wedge d G_{n}, \\
d x_{1}^{*} \wedge \cdots \wedge d x_{n}^{*} & =\frac{u_{1}-u_{2}}{x_{2}^{*}} d x_{1} \wedge \cdots \wedge d x_{n} .
\end{aligned}
$$

Hence 
Lemma 4.5. We have the Jacobian identities

$$
\begin{aligned}
& \text { (1) } \frac{\partial\left(G_{1}^{*}, \ldots, G_{n}^{*}\right)}{\partial\left(x_{1}, \ldots, x_{n}\right)} \equiv(-1)^{n-1} \frac{\partial\left(G_{1}, \ldots, G_{n}\right)}{\partial\left(x_{1}, \ldots, x_{n}\right)}\left(u_{1}-u_{2}\right)\left(f_{n+1}\right)^{n}\left(f_{n+2}\right)^{n-1} \prod_{j=1}^{n} f_{j}, \\
& \text { (2) } \frac{\partial\left(t, G_{2}^{*}, \ldots, G_{n}^{*}\right)}{\partial\left(x_{1}, x_{2}, \ldots, x_{n}\right)} \equiv-\frac{u_{1}-u_{2}}{f_{n+1}^{2}}, \\
& \text { (3) } \psi^{\prime}(t) \frac{\partial\left(t, G_{2}^{*}, \ldots, G_{n}^{*}\right)}{\partial\left(x_{1}, x_{2}, \ldots, x_{n}\right)} \equiv \frac{\partial\left(G_{1}^{*}, \ldots, G_{n}^{*}\right)}{\partial\left(x_{1}, \ldots, x_{n}\right)} .
\end{aligned}
$$

Definition 4.6. Define the discriminant associated with the system of critical points $\mathbf{c}_{j}$ by

$$
\text { Discr }:=\prod_{1 \leq j<k \leq n+1}\left(\zeta_{j}-\zeta_{k}\right)^{2}=(-1)^{\frac{n(n+1)}{2}} \mathcal{N}\left(\bar{\psi}^{\prime}(t)\right) .
$$

On the other hand the Hessian of $F$ is defined by the Jacobian

$$
\operatorname{Hess}(F):=\frac{\partial\left(G_{1}, \ldots, G_{n}\right)}{\partial\left(x_{1}, \ldots, x_{n}\right)} .
$$

We have the following equality as the second main theorem.

\section{Theorem 4.7.}

$$
\operatorname{Discr}=\mathcal{N}(\operatorname{Hess}(F))\left\{\mathcal{N}\left(f_{n+1}\right)\right\}^{n+2}\left\{\mathcal{N}\left(f_{n+2}\right)\right\}^{n-1} \prod_{j=1}^{n} \mathcal{N}\left(f_{j}\right) .
$$

Hence a pair of critical points meet each other if and only if $\mathcal{N}(\operatorname{Hess}(F))$ vanishes.

\section{Circle Arrangements (i), Product Formulae for Norms}

Let $n+1$ quadratic polynomials of real coefficients in $x=\left(x_{1}, \ldots, x_{n}\right)$ be given:

$$
f_{j}(x):=Q(x)+2 \sum_{k=1}^{n} \alpha_{j k} x_{k}+\alpha_{j 0} \quad(1 \leq j \leq n+1),
$$

where $Q(x)$ denotes the quadratic form $\sum_{j=1}^{n} x_{j}^{2}$. They define the arrangement of hyperspheres $\mathcal{A}$ consisting of the hyperspheres $S_{j}: f_{j}=0$. The center $O_{j}$ and the radius $r_{j}\left(r_{j}>0\right)$ of $S_{j}$ satisfy

$$
O_{j}=-\left(\alpha_{j 1}, \ldots, \alpha_{j n}\right), \quad r_{j}^{2}=-\alpha_{j 0}+\sum_{k=1}^{n} \alpha_{j k}^{2} .
$$

We denote the distance between $O_{j}, O_{k}(j \neq k)$ by $\rho_{j k}\left(\rho_{j k}>0\right)$ such that $\rho_{j k}^{2}=\sum_{l=1}^{n}\left(\alpha_{j l}-\alpha_{k l}\right)^{2}$. For the multiplicative function

$$
\Phi(x)=\prod_{j=1}^{n+1} f_{j}^{\lambda_{j}}(x)
$$


we consider the integral $J(\varphi)$ in $\S 3$. For generic exponents $\lambda$ one can prove that the dimension of $H_{\nabla}^{n}\left(X, \Omega^{\prime}\right)$ is equal to $2^{n+1}-1$. As the representatives of a basis of $H_{\nabla}^{n}\left(X, \Omega^{\circ}\right)$ one can choose the following $n$th degree forms

$$
\varphi_{J} d x_{1} \wedge \cdots \wedge d x_{n}, \quad \varphi_{J}:=\frac{1}{\prod_{j \in J} f_{j}}
$$

where $J$ ranges over the family of arbitrary (unordered) subsets of indices in $\{1,2, \ldots, n+1\}$. (See [4-7] for hypergeometric integrals associated with hypersphere arrangements.)

Cayley-Menger determinants are defined in the following way and play an important role in the sequel. Denote by $\rho_{\star j}=\rho_{j \star}$ the radius $r_{j}$ for $j \in\{1,2, \ldots, n+1\}$ or 0 for $j=\star$.

Definition 5.1. The determinant

$$
B\left(\begin{array}{cc}
0 & J \\
0 & K
\end{array}\right)=B\left(\begin{array}{llll}
0 & j_{1} & \cdots & j_{p} \\
0 & k_{1} & \cdots & k_{p}
\end{array}\right):=\left|\begin{array}{cccc}
0 & 1 & \cdots & 1 \\
1 & \rho_{j_{1} k_{1}}^{2} & \cdots & \rho_{j_{1} k_{p}} \\
\vdots & \vdots & \ddots & \vdots \\
1 & \rho_{j_{p} k_{1}}^{2} & \cdots & \rho_{j_{p} k_{p}}^{2}
\end{array}\right|
$$

is called the "Cayley-Menger determinant" associated with $\mathcal{A}$, where $J=\left\{j_{1}, \ldots, j_{p}\right\}$ and $K=$ $\left\{k_{1}, \ldots, k_{p}\right\}$ denote two subsets of the indices in $\{\star, 1, \ldots, n+1\}$. In case when $J=K$ we simply write $B(0 J)$ instead of $B\left(\begin{array}{ll}0 & J \\ 0 & K\end{array}\right)$. Notice that

$$
B(0 j k)=2 \rho_{j k}^{2}>0, \quad B(0 \star j)=2 r_{j}^{2}>0 .
$$

For simplicity we restrict ourselves to the case $n=2$, so that $\mathcal{A}$ is the arrangement of three circles $S_{1}, S_{2}, S_{3}$ in $\mathbb{R}^{2}$. We further assume that $r_{j}$ equals the same simply denoted by $r$ and that $v_{j}=1$ for all $j$. One sees that

$$
\begin{aligned}
B\left(\begin{array}{lll}
0 & j & l \\
0 & k & l
\end{array}\right) & =\rho_{j l}^{2}+\rho_{k l}^{2}-\rho_{j k}^{2}, \quad B(0 \star j k)=\rho_{j k}^{2}\left(\rho_{j k}^{2}-4 r^{2}\right), \\
B(0123) & =\rho_{12}^{4}+\rho_{13}^{4}+\rho_{23}^{4}-2 \rho_{12}^{2} \rho_{13}^{2}-2 \rho_{12}^{2} \rho_{23}^{2}-2 \rho_{13}^{2} \rho_{23}^{2} \\
& =-\left(\rho_{13}+\rho_{23}-\rho_{12}\right)\left(\rho_{12}+\rho_{23}-\rho_{13}\right)\left(\rho_{12}+\rho_{13}-\rho_{23}\right)\left(\rho_{12}+\rho_{13}+\rho_{23}\right), \\
B(0 \star 123) & =-2 r^{2} B(0123)-2 \rho_{12}^{2} \rho_{13}^{2} \rho_{23}^{2} .
\end{aligned}
$$

In this case $F$ can be simply written

$$
F=\log f_{1}+\log f_{2}+\log f_{3} .
$$

We further assume the following condition of non-degeneracy of $\mathcal{A}$ :

$$
\left(C_{2}\right) \quad B(0123)<0, \quad B(0 \star 123) \neq 0, \quad B(0 \star j k) \neq 0 \quad(1 \leq j<k \leq 3),
$$

which means the triangle $\triangle \mathrm{O}_{1} \mathrm{O}_{2} \mathrm{O}_{3}$ is non-degenerate, i.e., any two circles have no contact point and three circles $S_{1}, S_{2}, S_{3}$ have no common point. By taking a suitable choice of coordinates we may assume that

$$
\alpha_{31}=\alpha_{32}=\alpha_{22}=0, \quad \alpha_{21}>0, \quad \alpha_{12}>0
$$


so that we have

$$
\begin{aligned}
& r^{2}=-\alpha_{30}=-\alpha_{20}+\alpha_{21}^{2}=-\alpha_{10}+\alpha_{11}^{2}+\alpha_{12}^{2}, \\
& \alpha_{21}^{2}=\rho_{23}^{2}, \quad \alpha_{11}^{2}+\alpha_{12}^{2}=\rho_{13}^{2}, \quad\left(\alpha_{11}-\alpha_{21}\right)^{2}+\alpha_{12}^{2}=\rho_{12}^{2}, \\
& 4 \alpha_{21}^{2} \alpha_{12}^{2}=-B(0123) .
\end{aligned}
$$

Hence $\alpha_{j k}$ are completely determined by $\rho_{j k}^{2}, r^{2}$.

Under the condition $\left(C_{2}\right)$ the system of equations (3.1) are equivalent to

$$
\begin{aligned}
G_{1} & :=\frac{x_{1}+\alpha_{11}}{f_{1}}+\frac{x_{1}+\alpha_{21}}{f_{2}}+\frac{x_{1}}{f_{3}}=0, \\
G_{2} & :=\frac{x_{2}+\alpha_{12}}{f_{1}}+\frac{x_{2}}{f_{2}}+\frac{x_{2}}{f_{3}}=0 .
\end{aligned}
$$

Generally there exist 7 (real or complex) points in $X$ satisfying (5.1) denoted by $\mathbf{c}_{j}(1 \leq j \leq 7$ ). Let $D_{j}(1 \leq j \leq 3)$ be the open disc surrounded by the circumference $\mathfrak{R e} S_{j}$. We notice that, if we consider the condition

$$
B(0 \star 123)>0, \quad B(0 \star j k)<0(1 \leq j<k \leq 3),
$$

then the intersection $D_{1} \cap D_{2} \cap D_{3}$ is not empty, and the critical points are all real and contained one by one in each compact chamber.

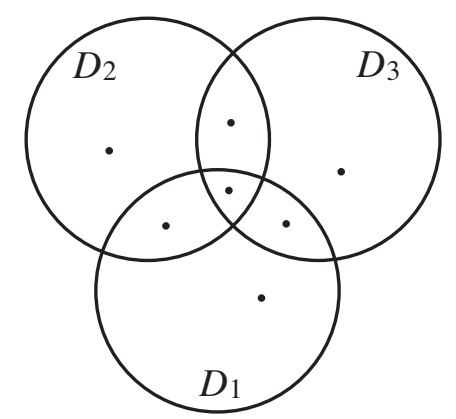

Figure 1: Critical points in compact chambers under the condition (5.2)

We introduce the new polynomials in $x$

$$
\begin{aligned}
& g_{1}:=f_{3}\left(L_{12}-L_{23}\right)-L_{23}\left(f_{1}-f_{3}\right), \\
& g_{2}:=f_{3}\left(L_{12}-L_{13}\right)-L_{13}\left(f_{2}-f_{3}\right), \\
& g_{3}:=-\left(L_{12}-L_{13}\right) L_{23}\left(f_{1}-f_{3}\right)+\left(L_{12}-L_{23}\right) L_{13}\left(f_{2}-f_{3}\right),
\end{aligned}
$$

where $L_{j k}$ denote linear functions of $x$

$$
\begin{aligned}
& L_{12}: L_{12}(x)=\alpha_{12} x_{1}+\left(-\alpha_{11}+\alpha_{21}\right) x_{2}+\alpha_{21} \alpha_{12}, \\
& L_{13}: L_{13}(x)=-\alpha_{12} x_{1}+\alpha_{11} x_{2}, \\
& L_{23}: L_{23}(x)=-\alpha_{21} x_{2} .
\end{aligned}
$$




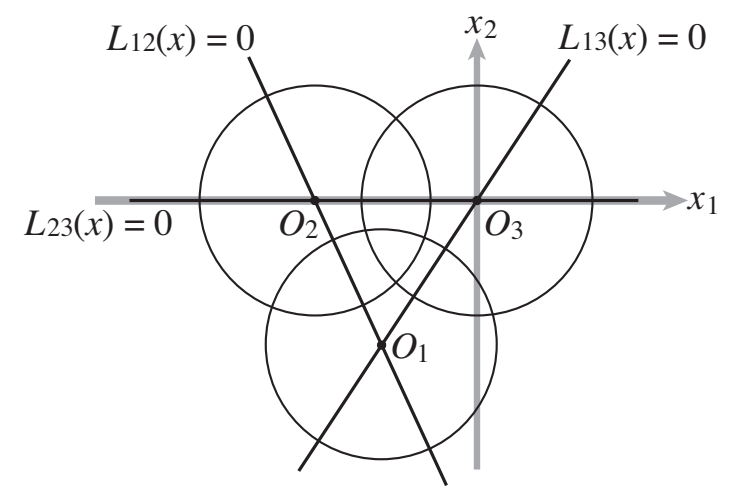

Figure 2: Points $O_{1}, O_{2}, O_{3}$ and Lines $L_{12}(x), L_{13}(x), L_{23}(x)=0$

Among $g_{1}, g_{2}, g_{3}$ there is the following identity

$$
g_{3}=\left(L_{12}-L_{13}\right) g_{1}-\left(L_{12}-L_{23}\right) g_{2} .
$$

$L_{j k}(x)=0$ defines the straight line going through $O_{j}, O_{k}$ and the triangle $\triangle O_{1} O_{2} O_{3}$ is defined by $L_{j k} \geq 0$.

Lemma 5.2. Under the condition $\left(C_{2}\right)$ the system of equations (5.1) are equivalent to the system

$$
g_{1}=g_{2}=g_{3}=0 \text {. }
$$

Suppose moreover that $\rho_{12} \neq \rho_{13}$ (or $\left.\rho_{12} \neq \rho_{23}\right)$. Then (5.4) is equivalent to the following system

$$
g_{2}=g_{3}=0 \quad\left(\text { or } g_{1}=g_{3}=0\right) \text {. }
$$

Case 1. Introduce the new parameters $t_{1}=f_{3} / f_{1}, t_{2}=f_{3} / f_{2}$ and denote $t_{\infty}=1+t_{1}+t_{2}$. We call $t_{1}, t_{2}$ "admissible coordinates" and $t_{1}$ "basic parameter". We want to find a rational curve $t_{2}=\omega\left(t_{1}\right) \subset X$ passing through all critical points $\mathbf{c}_{j}$ and a monic polynomial $\bar{\psi}\left(t_{1}\right)$ of degree 7 such that $\left(t_{1}, \omega\left(t_{1}\right)\right)_{\mathbf{c}_{j}}$ coincides with all $\left(t_{1}, t_{2}\right)$-coordinates of $\mathbf{c}_{j}$ for any root of $\bar{\psi}\left(t_{1}\right)$. In the sequel we shall call $\bar{\psi}\left(t_{1}\right)$ "characteristic polynomial".

To find out the characteristic polynomial we use Sylvester's elimination method and find the corresponding resultant $R=R\left(t_{1}\right)$. Namely we find two polynomials $U, V$ in $t_{1}$ such that $U t_{2}-V$ vanishes at all the critical points $\mathbf{c}_{j}(1 \leq j \leq 7)$ and put $\omega\left(t_{1}\right)=V / U$ (see Lemma 5.3 in more details. Refer to $[10,13])$. (5.4) gives the following congruences

$$
x_{1} \equiv-\frac{\alpha_{11} t_{1}+\alpha_{21} t_{2}}{t_{\infty}}, \quad x_{2} \equiv-\frac{\alpha_{12} t_{1}}{t_{\infty}},
$$

and conversely

$$
t_{1} \equiv \frac{L_{23}}{L_{12}}, \quad t_{2} \equiv \frac{L_{13}}{L_{12}}, \quad t_{\infty}=\frac{\alpha_{21} \alpha_{12}}{L_{12}} .
$$

Then (5.4) can be rewritten using the parameters $t_{1}, t_{2}$ as

$$
\tilde{g}_{1}=\tilde{g}_{2}=\tilde{g}_{3}=0,
$$


respectively where

$$
\tilde{g}_{1}:=g_{1} \frac{t_{\infty}^{3}}{\alpha_{21} \alpha_{12}}, \quad \tilde{g}_{2}:=g_{2} \frac{t_{\infty}^{3}}{\alpha_{21} \alpha_{12}}, \quad \tilde{g}_{3}:=g_{3} \frac{t_{\infty}^{3}}{\alpha_{21}^{2} \alpha_{12}^{2}} .
$$

It holds the following relation equivalent to (5.3):

$$
t_{\infty} \tilde{g}_{3}=\left(1-t_{2}\right) \tilde{g}_{1}-\left(1-t_{1}\right) \tilde{g}_{2} .
$$

$\tilde{g}_{1}, \tilde{g}_{2}, \tilde{g}_{3}$ are polynomials of third degree in $t_{1}, t_{2}$ as follows

$$
\tilde{g}_{1}=a_{0} t_{2}^{2}+a_{1} t_{2}+a_{2}, \quad \tilde{g}_{2}=b_{0} t_{2}^{3}+b_{1} t_{2}^{2}+b_{2} t_{2}+b_{3}, \quad \tilde{g}_{3}=c_{0} t_{2}^{2}+c_{1} t_{2}+c_{2},
$$

where $a_{j}, b_{k}, c_{l}$ are given by polynomials in $t_{1}$ :

$$
\begin{aligned}
& a_{0}=\left(r^{2}-\rho_{12}^{2}\right) t_{1}+\rho_{23}^{2}-r^{2}, \\
& a_{1}=2\left\{r^{2} t_{1}^{2}+\left(\rho_{23}^{2}-\rho_{12}^{2}\right) t_{1}-r^{2}\right\}, \\
& a_{2}=\left(t_{1}-1\right)\left\{r^{2} t_{1}^{2}+\left(\rho_{13}^{2}+2 r^{2}\right) t_{1}+r^{2}\right\}, \\
& b_{0}=r^{2}, \\
& b_{1}=2 r^{2} t_{1}+\rho_{23}^{2}+r^{2}, \\
& b_{2}=\left(r^{2}-\rho_{12}^{2}\right) t_{1}^{2}+2\left(\rho_{13}^{2}-\rho_{12}^{2}\right) t_{1}-\left(r^{2}+\rho_{23}^{2}\right), \\
& b_{3}=\left(\rho_{13}^{2}-r^{2}\right) t_{1}^{2}-2 r^{2} t_{1}-r^{2}, \\
& c_{0}=\rho_{12}^{2} t_{1}-\rho_{23}^{2}, \\
& c_{1}=-\rho_{12}^{2} t_{1}^{2}+\rho_{23}^{2}, \\
& c_{2}=\rho_{13}^{2} t_{1}\left(t_{1}-1\right) .
\end{aligned}
$$

Notice that $\tilde{g}_{j}=\tilde{g}_{j}\left(t_{1}, t_{2}\right)(1 \leq j \leq 3)$ satisfy

$$
\begin{aligned}
& \tilde{g}_{1}\left(t_{1}, 1\right)=a_{0}+a_{1}+a_{2}=r^{2} t_{1}^{3}+\left(\rho_{13}^{2}+3 r^{2}\right) t_{1}^{2}+\left(2 \rho_{23}^{2}-3 \rho_{12}^{2}-\rho_{13}^{2}\right) t_{1}+\rho_{23}^{2}-4 r^{2}, \\
& \tilde{g}_{2}\left(t_{1}, 1\right)=b_{0}+b_{1}+b_{2}+b_{3}=\left(\rho_{13}^{2}-\rho_{12}^{2}\right) t_{1}\left(t_{1}+2\right), \\
& \tilde{g}_{3}\left(t_{1}, 1\right)=c_{0}+c_{1}+c_{2}=\left(\rho_{13}^{2}-\rho_{12}^{2}\right) t_{1}\left(t_{1}-1\right) .
\end{aligned}
$$

As an immediate consequence we have

\section{Lemma 5.3.}

$$
\begin{aligned}
& \tilde{g}_{1}(0,1)=\rho_{23}^{2}-4 r^{2}, \quad \tilde{g}_{2}(0,1)=\tilde{g}_{3}(0,1)=0, \\
& \tilde{g}_{2}(1,0)=\rho_{13}^{2}-4 r^{2}, \quad \tilde{g}_{1}(1,0)=\tilde{g}_{3}(1,0)=0, \\
& \tilde{g}_{1}(0,-1)=\rho_{23}^{2}, \quad \tilde{g}_{2}(0,-1)=2 \rho_{23}^{2}, \quad \tilde{g}_{3}(0,-1)=-2 \rho_{23}^{2} \\
& \tilde{g}_{1}(-1,0)=2 \rho_{13}^{2}, \quad \tilde{g}_{2}(-1,0)=\rho_{13}^{2}, \quad \tilde{g}_{3}(-1,0)=2 \rho_{13}^{2} .
\end{aligned}
$$

The following Lemma is a simple application of Sylvester's elimination method (see [8, 10, 13]). 
Lemma 5.4. Put

$$
\begin{aligned}
& U: U\left(t_{1}\right)=b_{0}\left(c_{1}^{2}-c_{0} c_{2}\right)-b_{1} c_{0} c_{1}+b_{2} c_{0}^{2}, \\
& V: V\left(t_{1}\right)=-b_{0} c_{1} c_{2}+b_{1} c_{0} c_{2}-b_{3} c_{0}^{2} .
\end{aligned}
$$

Then the following identity holds:

$$
\tilde{g}_{23}:=c_{0}^{2} \tilde{g}_{2}-\left(b_{0} c_{0} t_{2}+b_{1} c_{0}-b_{0} c_{1}\right) \tilde{g}_{3}=U t_{2}-V \text { for arbitrary } t_{1}, t_{2} .
$$

If $\tilde{g}_{2}=\tilde{g}_{3}=0$, then $\tilde{g}_{23}=0$. This implies

$$
t_{2} \equiv \omega\left(t_{1}\right), \quad \omega\left(t_{1}\right):=\frac{V}{U}
$$

The resultant $R$ of $\tilde{g}_{2}\left(t_{1}, t_{2}\right)$ and $\tilde{g}_{3}\left(t_{1}, t_{2}\right)$ relative to $t_{2}$ is a polynomial in $t_{1}$ of degree 8 written by Sylvester determinant

$$
R: R\left(t_{1}\right)=\left|\begin{array}{lllll}
b_{0} & b_{1} & b_{2} & b_{3} & \\
& b_{0} & b_{1} & b_{2} & b_{3} \\
c_{0} & c_{1} & c_{2} & & \\
& c_{0} & c_{1} & c_{2} & \\
& & c_{0} & c_{1} & c_{2}
\end{array}\right|
$$

which is related to $U, V$ and can be described as follows:

$$
c_{0}^{2} R=U^{2} \psi\left(t_{1}\right)=c_{0} V^{2}+c_{1} V U+c_{2} U^{2},
$$

where $U, V$ are polynomials of degree 4 which can be written as

$$
U=\sum_{j=0}^{4} u_{j} t_{1}^{4-j}, \quad V=\sum_{j=0}^{4} v_{j} t_{1}^{4-j} .
$$

In particular the coefficients $u_{0}, u_{4}$ and $v_{0}, v_{4}$ are given by

$$
u_{0}=-\left(\rho_{12}^{2}-4 r^{2}\right) \rho_{12}^{4}, \quad u_{4}=r^{2} \rho_{23}^{4}, \quad v_{0}=\rho_{12}^{2}\left\{r^{2}\left(\rho_{12}^{2}+3 \rho_{13}^{2}\right)-\rho_{2}^{2} \rho_{13}^{2}\right\}, \quad v_{4}=r^{2} \rho_{23}^{4} .
$$

From (5.10) and (5.14), $\psi\left(t_{1}\right)$ is expressed as

$$
\psi\left(t_{1}\right)=c_{0}\left(\frac{V}{U}\right)^{2}+c_{1} \frac{V}{U}+c_{2}=\tilde{g}_{3}\left(t_{1}, \omega\left(t_{1}\right)\right)
$$

Moreover $U-V$ can be evaluated explicitly from (5.13)

$$
\begin{aligned}
U-V & =c_{0}^{2} \tilde{g}_{2}\left(t_{1}, 1\right)-\left(b_{0} c_{0}+b_{1} c_{0}-b_{0} c_{1}\right) \tilde{g}_{3}\left(t_{1}, 1\right)=\left(\rho_{13}^{2}-\rho_{12}^{2}\right) W^{*}, \\
W^{*} & =t_{1}\left(w_{0} t_{1}^{3}+w_{1} t_{1}^{2}+w_{2} t_{1}+w_{3}\right)
\end{aligned}
$$

such that

$$
\begin{aligned}
& w_{0}=\rho_{12}^{2}\left(\rho_{12}^{2}-3 r^{2}\right), \quad w_{1}=-\rho_{12}^{2}\left(3 \rho_{23}^{2}-2 \rho_{12}^{2}\right)+\left(2 \rho_{23}^{2}+\rho_{12}^{2}\right) r^{2}, \\
& w_{2}=\rho_{23}^{2}\left(2 \rho_{23}^{2}-3 \rho_{12}^{2}\right)+\left(2 \rho_{12}^{2}+\rho_{23}^{2}\right) r^{2}, \quad w_{3}=\rho_{23}^{2}\left(\rho_{23}^{2}-3 r^{2}\right) .
\end{aligned}
$$

$R$ is a polynomial in $t_{1}$ of degree 8 and a polynomial in $\rho_{j k}^{2}, r^{2}$ of degree 10 . 
Lemma 5.5. (1) If $\rho_{12}^{2}=\rho_{13}^{2}$, then $R\left(t_{1}\right)$ vanishes.

(2) $R(0)$ always vanishes.

Proof. About (1). If $\rho_{12}^{2}=\rho_{13}^{2}, U$ coincides with $V$, so that

$$
c_{0}^{2} R\left(t_{1}\right)=\left(c_{0}+c_{1}+c_{2}\right) U^{2}=0 .
$$

(5.11) shows that $c_{0}$ does not vanish for $\rho_{13}^{2}=\rho_{12}^{2}$. This implies $R\left(t_{1}\right)=0$.

About (2). From Lemma 5.3 the identity $U(0)=V(0)$ holds true. Hence

$$
-\rho_{23}^{2} R(0)=\left(c_{0}(0)+c_{1}(0)+c_{2}(0)\right) U(0)=0
$$

because of (5.12).

Owing to Lemma $5.5 R\left(t_{1}\right)$ has the factor $\left(\rho_{12}^{2}-\rho_{13}^{2}\right) t_{1}$. On the other hand since

$$
c_{0} \approx \rho_{12}^{2} t_{1}, \quad c_{1} \approx-\rho_{12}^{2} t_{1}^{2}, \quad c_{2} \approx \rho_{13}^{2} t_{1}^{2} \quad\left(\left|t_{1}\right| \uparrow \infty\right)
$$

in view of the formulae above for $u_{0}, v_{0}$, we have

$$
R\left(t_{1}\right) \approx r^{2} \rho_{12}^{4}\left(\rho_{12}^{2}-4 r^{2}\right)\left(\rho_{12}^{2}-\rho_{13}^{2}\right) t_{1}^{8} \quad\left(\left|t_{1}\right| \uparrow \infty\right) .
$$

As a result

Lemma 5.6. $R\left(t_{1}\right)$ is a polynomial in $t_{1}$ of degree 8 and a polynomial in $\rho_{j k}^{2}, r^{2}$ of degree 5 with the factor $\left(\rho_{12}^{2}-\rho_{13}^{2}\right) t_{1}$ such that

$$
\begin{aligned}
& R\left(t_{1}\right)=r^{2} \rho_{12}^{4}\left(\rho_{12}^{2}-4 r^{2}\right)\left(\rho_{12}^{2}-\rho_{13}^{2}\right) t_{1} \bar{\psi}\left(t_{1}\right), \\
& R\left(t_{1}\right) \approx-r^{2} \rho_{23}^{4}\left(\rho_{23}^{2}-4 r^{2}\right)\left(\rho_{12}^{2}-\rho_{13}^{2}\right) t_{1} \quad\left(t_{1} \downarrow 0\right),
\end{aligned}
$$

where $\bar{\psi}\left(t_{1}\right)=\prod_{j=1}^{7}\left(t_{1}-\zeta_{j}\right)$ is a monic polynomial with 7 roots $\zeta_{j}(1 \leq j \leq 7)$ such that

$$
-\bar{\psi}(0)=\prod_{j=1}^{7} \zeta_{j}=\frac{\rho_{23}^{4}\left(\rho_{23}^{2}-4 r^{2}\right)}{\rho_{12}^{4}\left(\rho_{12}^{2}-4 r^{2}\right)}=\frac{\rho_{23}^{2} B(0 \star 23)}{\rho_{12}^{2} B(0 \star 12)} .
$$

$\bar{\psi}\left(t_{1}\right)$ is the characteristic polynomial relative to the basic parameter $t_{1}$ of the critical points $\mathbf{c}_{j}$ such that $t_{1}\left(\mathbf{c}_{j}\right)=\zeta_{j}$.

Furthermore since

$$
\begin{aligned}
U(1) & =\left(\rho_{23}^{2}-\rho_{13}^{2}\right)^{2}\left(4 r^{2}+2 \rho_{13}^{2}-3 \rho_{12}^{2}\right), \\
V(1) & =-\left(\rho_{23}^{2}-\rho_{12}^{2}\right)^{2}\left(\rho_{13}^{2}-4 r^{2}\right), \\
U(1)-V(1) & =3\left(\rho_{23}^{2}-\rho_{12}^{2}\right)^{2}\left(\rho_{13}^{2}-\rho_{12}^{2}\right),
\end{aligned}
$$

we have the formula

$$
R(1)=-3\left(\rho_{23}^{2}-\rho_{12}^{2}\right)^{3}\left(\rho_{13}^{2}-\rho_{12}^{2}\right)\left(\rho_{13}^{2}-4 r^{2}\right)
$$

Hence

$$
\bar{\psi}(1)=\prod_{j=1}^{7}\left(1-\zeta_{j}\right)=-3 \frac{\left(\rho_{12}^{2}-\rho_{23}^{2}\right)^{3}\left(\rho_{13}^{2}-4 r^{2}\right)}{\rho_{12}^{4} r^{2}\left(\rho_{12}^{2}-4 r^{2}\right)} .
$$

Seeing that $\left(f_{1}-f_{3}\right) / f_{1}=1-t_{1},\left(f_{2}-f_{3}\right) / f_{2}=1-t_{2}$ we can conclude 
Proposition 5.7. (1)

$$
\begin{aligned}
& \mathcal{N}\left(t_{1}\right)=\mathcal{N}\left(\frac{f_{3}}{f_{1}}\right)=\frac{\rho_{23}^{2} B(0 \star 23)}{\rho_{12}^{2} B(0 \star 12)} . \\
& \mathcal{N}\left(t_{2}\right)=\mathcal{N}\left(\frac{f_{3}}{f_{2}}\right)=\frac{\rho_{13}^{2} B(0 \star 13)}{\rho_{12}^{2} B(0 \star 12)} .
\end{aligned}
$$

(2)

$$
\begin{aligned}
& \mathcal{N}\left(1-t_{1}\right)=\mathcal{N}\left(\frac{f_{1}-f_{3}}{f_{1}}\right)=-3 \frac{\left(\rho_{12}^{2}-\rho_{23}^{2}\right)^{3} B(0 \star 13)}{\rho_{12}^{2} \rho_{13}^{2} r^{2} B(0 \star 12)} \\
& \mathcal{N}\left(1-t_{2}\right)=\mathcal{N}\left(\frac{f_{2}-f_{3}}{f_{2}}\right)=-3 \frac{\left(\rho_{12}^{2}-\rho_{13}^{2}\right)^{3} B(0 \star 23)}{\rho_{12}^{2} \rho_{23}^{2} r^{2} B(0 \star 12)}
\end{aligned}
$$

Case 2. Instead of $\left(t_{1}, t_{2}\right)$ we now take the new coordinates $\left(t_{\infty}, t_{1}\right)$, where $t_{\infty}$ being the basic parameter. By the substitution $t_{2}=t_{\infty}-t_{1}-1, \tilde{g}_{2}, \tilde{g}_{3}$ and $2 \tilde{g}_{2}-\tilde{g}_{3}$ can be rewritten in terms of $t_{\infty}, t_{1}$ as

$$
\begin{aligned}
& \tilde{g}_{2}^{\sharp}\left(t_{\infty}, t_{1}\right):=\tilde{g}_{2}\left(t_{1}, t_{\infty}-t_{1}-1\right)=b_{0}^{\prime} t_{1}^{3}+b_{1}^{\prime} t_{1}^{2}+b_{2}^{\prime} t_{1}+b_{3}^{\prime}, \\
& \tilde{g}_{3}^{\sharp}\left(t_{\infty}, t_{1}\right):=\tilde{g}_{3}\left(t_{1}, t_{\infty}-t_{1}-1\right), \\
& \tilde{g}_{23}^{\sharp}\left(t_{\infty}, t_{1}\right):=2 \tilde{g}_{2}^{\sharp}\left(t_{\infty}, t_{1}\right)-\tilde{g}_{3}^{\sharp}\left(t_{\infty}, t_{1}\right)=c_{0}^{\prime} t_{1}^{2}+c_{1}^{\prime} t_{1}+c_{2}^{\prime},
\end{aligned}
$$

where $b_{0}^{\prime}, b_{1}^{\prime}, b_{2}^{\prime}, b_{3}^{\prime} ; c_{0}^{\prime}, c_{1}^{\prime}, c_{2}^{\prime}$ denote polynomials in $t_{\infty}$ as follows:

$$
\begin{aligned}
& b_{0}^{\prime}=\rho_{12}^{2}, \\
& b_{1}^{\prime}=-\rho_{12}^{2} t_{\infty}+\rho_{23}^{2}-\rho_{13}^{2}+3 \rho_{12}^{2}, \\
& b_{2}^{\prime}=-r^{2} t_{\infty}^{2}+2\left(-\rho_{12}^{2}+\rho_{13}^{2}-\rho_{23}^{2}\right) t_{\infty}+\left(2 \rho_{12}^{2}-2 \rho_{13}^{2}+3 \rho_{23}^{2}\right), \\
& b_{3}^{\prime}=\left(t_{\infty}-2\right)\left\{r^{2} t_{\infty}^{2}+\rho_{23}^{2}\left(t_{\infty}-1\right)\right\}, \\
& c_{0}^{\prime}=c_{00}^{\prime} t_{\infty}+c_{01}^{\prime}, \quad c_{1}^{\prime}=c_{10}^{\prime} t_{\infty}^{2}+c_{11}^{\prime} t_{\infty}+c_{12}^{\prime}, \quad c_{2}^{\prime}=c_{20}^{\prime} t_{\infty}^{3}+c_{21}^{\prime} t_{\infty}^{2}+c_{22}^{\prime} t_{\infty}+c_{23}^{\prime}
\end{aligned}
$$

such that

$$
\begin{aligned}
& c_{00}^{\prime}=\rho_{12}^{2}, \quad c_{01}^{\prime}=3\left(\rho_{23}^{2}-\rho_{13}^{2}+\rho_{12}^{2}\right), \\
& c_{10}^{\prime}=-\left(2 r^{2}+\rho_{12}^{2}\right), \quad c_{11}^{\prime}=4 \rho_{13}^{2}-2 \rho_{12}^{2}-6 \rho_{23}^{2}, \quad c_{12}^{\prime}=3\left(\rho_{12}^{2}-\rho_{13}^{2}+3 \rho_{23}^{2}\right), \\
& c_{20}^{\prime}=2 r^{2}, \quad c_{21}^{\prime}=3 \rho_{23}^{2}-4 r^{2}, \quad c_{22}^{\prime}=-9 \rho_{23}^{2}, \quad c_{23}^{\prime}=6 \rho_{23}^{2} .
\end{aligned}
$$

Then like Lemma 5.4 the following holds.

Lemma 5.8. Put

$$
\begin{aligned}
& U^{\sharp}: U^{\sharp}\left(t_{\infty}\right)=b_{0}^{\prime}\left(c_{1}^{\prime 2}-c_{0}^{\prime} c_{2}^{\prime}\right)-b_{1}^{\prime} c_{0}^{\prime} c_{1}^{\prime}+b_{2}^{\prime} c_{0}^{\prime 2}, \\
& V^{\sharp}: V^{\sharp}\left(t_{\infty}\right)=-b_{0}^{\prime} c_{1}^{\prime} c_{2}^{\prime}+b_{1}^{\prime} c_{0}^{\prime} c_{2}^{\prime}-b_{3}^{\prime} c_{0}^{\prime 2} .
\end{aligned}
$$

Then

$$
0 \equiv c_{0}^{\prime 2} \tilde{g}_{2}^{\sharp}-\left(b_{0}^{\prime} c_{0}^{\prime} t_{1}+b_{1}^{\prime} c_{0}^{\prime}-b_{0}^{\prime} c_{1}^{\prime}\right) \tilde{g}_{23}^{\sharp}=U^{\sharp} t_{1}-V^{\sharp},
$$


i.e., the rational curve $t_{1}=V^{\sharp}\left(t_{\infty}\right) / U^{\sharp}\left(t_{\infty}\right)$ gives the interpolating curve relative to the admissible coordinates $\left(t_{\infty}, t_{1}\right)$. We have

$$
U^{\sharp}=\sum_{j=0}^{4} u_{j}^{\prime} t_{\infty}^{4-j}, \quad V^{\sharp}=\sum_{j=0}^{5} v_{j}^{\prime} t_{\infty}^{5-j}
$$

with

$$
u_{0}^{\prime}=v_{0}^{\prime}=r^{2} \rho_{12}^{2}\left(4 r^{2}-\rho_{12}^{2}\right), \quad u_{1}^{\prime}-v_{1}^{\prime}=2 r^{2} \rho_{12}^{2}\left(4 r^{2}-\rho_{13}^{2}\right),
$$

so that

$$
\frac{V^{\sharp}}{U^{\sharp}} \approx t_{\infty}+\frac{v_{1}^{\prime}-u_{1}^{\prime}}{u_{0}^{\prime}}+O\left(\frac{1}{t_{\infty}}\right) \quad\left(\left|t_{\infty}\right| \uparrow \infty\right) .
$$

For $t_{\infty}$ being fixed, the resultant $R^{\sharp}=R^{\sharp}\left(t_{\infty}\right)$ of $\tilde{g}_{2}^{\sharp}$ and $\tilde{g}_{23}^{\sharp}$ relative to $t_{1}$ is given by

$$
R^{\sharp}:=\left|\begin{array}{ccccc}
b_{0}^{\prime} & b_{1}^{\prime} & b_{2}^{\prime} & b_{3}^{\prime} & \\
& b_{0}^{\prime} & b_{1}^{\prime} & b_{2}^{\prime} & b_{3}^{\prime} \\
c_{0}^{\prime} & c_{1}^{\prime} & c_{2}^{\prime} & & \\
& c_{0}^{\prime} & c_{1}^{\prime} & c_{2}^{\prime} & \\
& & c_{0}^{\prime} & c_{1}^{\prime} & c_{2}^{\prime}
\end{array}\right|,
$$

which satisfies the identity

$$
c_{0}^{\prime 2} R^{\sharp}=c_{0}^{\prime} V^{\sharp^{2}}+c_{1}^{\prime} U^{\sharp} V^{\sharp}+c_{2}^{\prime} U^{\sharp^{2}} .
$$

As a result of Lemma 5.8, we have

$$
c_{0}^{\prime 2} R^{\sharp} \approx u_{0}^{\prime}\left\{u_{0}^{\prime}\left(c_{01}^{\prime}+c_{11}^{\prime}+c_{21}^{\prime}\right)+\left(v_{1}^{\prime}-u_{1}^{\prime}\right)\left(2 c_{00}^{\prime}+c_{10}^{\prime}\right)\right\} t_{\infty}^{8} \quad\left(\left|t_{\infty}\right| \uparrow \infty\right) .
$$

Seeing that

$$
c_{0}^{\prime 2} \approx \rho_{12}^{4} t_{\infty}^{2} \quad\left(\left|t_{\infty}\right| \uparrow \infty\right), \quad c_{01}^{\prime}+c_{11}^{\prime}+c_{21}^{\prime}=-4 r^{2}+\rho_{12}^{2}+\rho_{13}^{2}, \quad 2 c_{00}^{\prime}+c_{10}^{\prime}=\rho_{12}^{2}-2 r^{2},
$$

a direct calculation using Lemma 5.8 shows

$$
u_{0}^{\prime}\left(c_{01}^{\prime}+c_{11}^{\prime}+c_{21}^{\prime}\right)+\left(v_{1}^{\prime}-u_{1}^{\prime}\right)\left(2 c_{00}^{\prime}+c_{10}^{\prime}\right)=r^{2} \rho_{12}^{4}\left(\rho_{13}^{2}-\rho_{12}^{2}\right)
$$

hence

$$
R^{\sharp}=-r^{4} \rho_{12}^{2}\left(\rho_{12}^{2}-4 r^{2}\right)\left(\rho_{13}^{2}-\rho_{12}^{2}\right) t_{\infty}^{8}\left(1+O\left(\frac{1}{t_{\infty}}\right)\right) .
$$

On the other hand (4.15) shows the equality

$$
\tilde{g}_{2}^{\sharp}(2,1)=\tilde{g}_{3}^{\sharp}(2,1)=0,
$$

i.e., the two polynomials $\tilde{g}_{2}^{\sharp}\left(2, t_{1}\right), \tilde{g}_{3}^{\sharp}\left(2, t_{1}\right)$ have a common zero at $t_{1}=1$, which means $R^{\sharp}(2)=0$. Hence $R^{\sharp}$ can be described more precisely as

$$
R^{\sharp}\left(t_{\infty}\right)=-r^{4} \rho_{12}^{2}\left(\rho_{12}^{2}-4 r^{2}\right)\left(\rho_{13}^{2}-\rho_{12}^{2}\right)\left(t_{\infty}-2\right) \prod_{j=1}^{7}\left(t_{\infty}-\zeta_{j}^{\prime}\right),
$$

where $\zeta_{j}^{\prime}$ denotes the value $t_{\infty}\left(\mathbf{c}_{j}\right)$. We notice that $\bar{\psi}\left(t_{\infty}\right)=\prod_{j=1}^{7}\left(t_{\infty}-\zeta_{j}^{\prime}\right)$ is the characteristic polynomial relative to the parameter $t_{\infty}$.

We now want to evaluate the value of $R^{\sharp}\left(t_{\infty}\right)$ at $t_{\infty}=0$. Before that one first sees the following identities hold. 


\section{Lemma 5.9.}

$$
\begin{aligned}
U^{\sharp}(0)+V^{\sharp}(0) & =-9 \rho_{13}^{2}\left(\rho_{23}^{2}+\rho_{12}^{2}-\rho_{13}^{2}\right)^{2}=-\rho_{13}^{2} c_{0}^{\prime}(0)^{2}, \\
V^{\sharp}(0) & =-18 \rho_{12}^{2} \rho_{23}^{2}\left(\rho_{13}^{2}+\rho_{23}^{2}-\rho_{12}^{2}\right) .
\end{aligned}
$$

Proof. Notice first that

$$
\begin{aligned}
& \tilde{g}_{2}^{\sharp}(0,-1)=\tilde{g}_{2}(-1,0)=\rho_{13}^{2}, \quad \tilde{g}_{3}^{\sharp}(0,-1)=\tilde{g}_{3}(-1,0)=2 \rho_{13}^{2}, \\
& \tilde{g}_{23}^{\sharp}(0,-1)=2 \tilde{g}_{2}^{\sharp}(0,-1)-\tilde{g}_{3}^{\sharp}(0,-1)=0, \quad c_{0}^{\prime}(0)=3\left(\rho_{23}^{2}+\rho_{12}^{2}-\rho_{13}^{2}\right) .
\end{aligned}
$$

One may put $t_{\infty}=0, t_{1}=-1$ in (5.16). Then one has

$$
-U^{\sharp}(0)-V^{\sharp}(0)=c_{0}^{\prime}(0)^{2} \tilde{g}_{2}^{\sharp}(0,-1)=9 \rho_{13}^{2}\left(\rho_{23}^{2}+\rho_{12}^{2}-\rho_{13}^{2}\right)^{2} .
$$

On the other hand since $\tilde{g}_{2}^{\sharp}(0,0)=\tilde{g}_{2}(0,-1)=2 \rho_{23}^{2}, \tilde{g}_{3}^{\sharp}(0,0)=\tilde{g}_{3}(0,-1)=-2 \rho_{23}^{2}$, one has $\tilde{g}_{23}^{\sharp}(0,0)=6 \rho_{23}^{2}$. Furthermore

$$
b_{1}^{\prime}(0) c_{0}^{\prime}(0)-b_{0}^{\prime}(0) c_{1}^{\prime}(0)=3\left(\rho_{23}^{2}-\rho_{13}^{2}+\rho_{12}^{2}\right)\left(\rho_{23}^{2}-\rho_{13}^{2}+3 \rho_{12}^{2}\right)-3 \rho_{12}^{2}\left(\rho_{12}^{2}-\rho_{13}^{2}+3 \rho_{23}^{2}\right) .
$$

Hence

$$
-V^{\sharp}(0)=c_{0}^{\prime}(0)^{2} \tilde{g}_{2}^{\sharp}(0,0)-\left\{b_{1}^{\prime}(0) c_{0}^{\prime}(0)-b_{0}^{\prime}(0) c_{1}^{\prime}(0)\right\} \tilde{g}_{3}^{\sharp}(0,0)=18 \rho_{12}^{2} \rho_{23}^{2}\left(\rho_{23}^{2}+\rho_{13}^{2}-\rho_{12}^{2}\right) .
$$

Lemma 5.9 has thus been proved.

Due to Lemmas 5.8 and 5.9 we have

Lemma 5.10. The following identity holds:

$$
\begin{aligned}
R^{\sharp}(0) & =-2 r^{4} \rho_{12}^{2}\left(\rho_{12}^{2}-4 r^{2}\right)\left(\rho_{13}^{2}-\rho_{12}^{2}\right) \prod_{j=1}^{7} \zeta_{j}^{\prime} \\
& =54 \rho_{13}^{2} \rho_{23}^{2}\left(\rho_{13}^{2}-\rho_{12}^{2}\right) B(0123) .
\end{aligned}
$$

Proof. (5.19) is a direct consequence of (5.18). Since $c_{0}^{\prime}(0)-c_{1}^{\prime}(0)+c_{2}^{\prime}(0)=0$, the formula (5.17) of $R^{\sharp}\left(t_{\infty}\right)$ at $t_{\infty}=0$ in Lemma 5.8 becomes

$$
R^{\sharp}(0)=\frac{U^{\sharp}(0)+V^{\sharp}(0)}{c_{0}^{\prime}(0)^{2}}\left\{c_{2}^{\prime}(0)\left(U^{\sharp}(0)+V^{\sharp}(0)\right)+\left(c_{1}^{\prime}(0)-2 c_{2}^{\prime}(0)\right) V^{\sharp}(0)\right\} .
$$

Using Lemma 5.9 and $c_{1}^{\prime}(0)=3\left(\rho_{12}^{2}-\rho_{13}^{2}+3 \rho_{23}^{2}\right), c_{2}^{\prime}(0)=6 \rho_{23}^{2}$ we have $\left(U^{\sharp}(0)+V^{\sharp}(0)\right) / c_{0}^{\prime}(0)^{2}=$ $-\rho_{13}^{2}$ and obtain

$$
\begin{aligned}
c_{2}^{\prime}(0) & \left(U^{\sharp}(0)+V^{\sharp}(0)\right)+\left(c_{1}^{\prime}(0)-2 c_{2}^{\prime}(0)\right) V^{\sharp}(0) \\
& =-6 \rho_{23}^{2} \cdot 9 \rho_{13}^{2}\left(\rho_{23}^{2}+\rho_{12}^{2}-\rho_{13}^{2}\right)^{2}-3\left(\rho_{12}^{2}-\rho_{13}^{2}-\rho_{23}^{2}\right) \cdot 18 \rho_{12}^{2} \rho_{23}^{2}\left(\rho_{13}^{2}+\rho_{23}^{2}-\rho_{12}^{2}\right) \\
& =-54 \rho_{23}^{2}\left\{\rho_{13}^{2}\left(\rho_{23}^{2}+\rho_{12}^{2}-\rho_{13}^{2}\right)^{2}-\rho_{12}^{2}\left(\rho_{23}^{2}+\rho_{13}^{2}-\rho_{12}^{2}\right)^{2}\right\} \\
& =-54 \rho_{23}^{2}\left(\rho_{13}^{2}-\rho_{12}^{2}\right)\left(\rho_{12}^{4}+\rho_{13}^{4}+\rho_{23}^{4}-2 \rho_{12}^{2} \rho_{13}^{2}-2 \rho_{12}^{2} \rho_{23}^{2}-2 \rho_{13}^{2} \rho_{23}^{2}\right) \\
& =-54 \rho_{23}^{2}\left(\rho_{13}^{2}-\rho_{12}^{2}\right) B(0123),
\end{aligned}
$$

which implies (5.20). 
Comparing (5.19) with (5.20) in Lemma 5.10 we can evaluate the norm of $t_{\infty}$ as follows:

\section{Proposition 5.11.}

$$
\mathcal{N}\left(t_{\infty}\right)=\prod_{j=1}^{7} \zeta_{j}^{\prime}=-27 \frac{\rho_{13}^{2} \rho_{23}^{2} B(0123)}{r^{4} B(0 \star 12)} .
$$

From Propositions 5.7 and 5.11, the identity (5.7) derives the formula for $\mathcal{N}\left(L_{12}\right)$. In the same way by symmetry of isometry the followings also hold:

\section{Corollary 5.12.}

$$
\begin{aligned}
& \mathcal{N}\left(L_{12}\right)=\frac{1}{2^{7} 3^{3}} \frac{r^{4} B(0 \star 12)}{\rho_{13}^{2} \rho_{23}^{2}}\{-B(0123)\}^{\frac{5}{2}}, \\
& \mathcal{N}\left(L_{13}\right)=\frac{1}{2^{7} 3^{3}} \frac{r^{4} B(0 \star 13)}{\rho_{12}^{2} \rho_{23}^{2}}\{-B(0123)\}^{\frac{5}{2}}, \\
& \mathcal{N}\left(L_{23}\right)=\frac{1}{2^{7} 3^{3}} \frac{r^{4} B(0 \star 23)}{\rho_{12}^{2} \rho_{13}^{2}}\{-B(0123)\}^{\frac{5}{2}}
\end{aligned}
$$

Put $\psi\left(t_{1}\right)=\tilde{g}_{3}\left(t_{1}, \omega\left(t_{1}\right)\right)$ such that $R=U^{2} \psi\left(t_{1}\right) / c_{0}^{2}$.

Case 3. We take the admissible coordinates $\left(s, t_{1}\right)$ and the basic parameter $s=-T t_{1}-t_{2}$ for $T=\left(\rho_{13}^{2}-\rho_{12}^{2}\right) / \rho_{23}^{2}$. (5.5) implies

$$
f_{2}-f_{3}=2 \alpha_{21} x_{1}+\alpha_{21}^{2} \equiv \frac{\rho_{23}^{2}(s+1)}{t_{\infty}}
$$

By the substitution $t_{2}=-T t_{1}-s, \tilde{g}_{1}$ and $\tilde{g}_{3}$ can be rewritten in terms of $s, t_{1}$ as

$$
\begin{aligned}
\tilde{g}_{1}^{\mathrm{b}} & :=\tilde{g}_{1}\left(t_{1},-T t_{1}-s\right)=\hat{a}_{0} t_{1}^{3}+\hat{a}_{1} t_{1}^{2}+\hat{a}_{2} t_{1}+\hat{a}_{3}, \\
\tilde{g}_{3}^{b} & :=\tilde{g}_{3}\left(t_{1},-T t_{1}-s\right)=\hat{c}_{0} t_{1}^{3}+\hat{c}_{1} t_{1}^{2}+\hat{c}_{2} t_{1}+\hat{c}_{3}, \\
\tilde{g}_{13}^{b} & :=\hat{c}_{0} \tilde{g}_{1}^{\mathrm{b}}-\hat{a}_{0} \tilde{g}_{3}^{\mathrm{b}}=\hat{c}_{0}^{\prime} t_{1}^{2}+\hat{c}_{1}^{\prime} t_{1}+\hat{c}_{2}^{\prime},
\end{aligned}
$$

where $\hat{a}_{0}, \hat{a}_{1}, \hat{a}_{2}, \hat{a}_{3}, \hat{c}_{0}, \hat{c}_{1}, \hat{c}_{2}, \hat{c}_{3}, \hat{c}_{0}^{\prime}, \hat{c}_{1}^{\prime}, \hat{c}_{2}^{\prime}$ denote polynomials in $s$ as follows:

$$
\begin{aligned}
\hat{a}_{0}= & r^{2}(T-1)^{2}-\rho_{12}^{2} T^{2}, \\
\hat{a}_{1}= & 2\left\{r^{2}(T-1)-\rho_{12}^{2} T\right\} s+T^{2}\left(\rho_{23}^{2}-r^{2}\right)-2\left(\rho_{23}^{2}-\rho_{12}^{2}\right) T+r^{2}+\rho_{13}^{2}, \\
\hat{a}_{2}= & \left(r^{2}-\rho_{12}^{2}\right) s^{2}+2\left\{\left(\rho_{23}^{2}-r^{2}\right) T-\left(\rho_{23}^{2}-\rho_{12}^{2}\right)\right\} s+2 r^{2} T-r^{2}-\rho_{13}^{2}, \\
\hat{a}_{3}= & \rho_{23}^{2} s^{2}-r^{2}(s-1)^{2}, \\
& \hat{c}_{0}=\rho_{12}^{2} T(T+1), \quad \hat{c}_{1}=\rho_{12}^{2}(2 T+1) s+\rho_{13}^{2}-\rho_{23}^{2} T^{2}, \\
& \hat{c}_{2}=\rho_{12}^{2} s^{2}-2 \rho_{23}^{2} T s-\left(\rho_{23}^{2} T+\rho_{13}^{2}\right), \quad \hat{c}_{3}=-\rho_{23}^{2} s(s+1),
\end{aligned}
$$

and

$$
\hat{c}_{0}^{\prime}=\hat{c}_{0} \hat{a}_{1}-\hat{a}_{0} \hat{c}_{1}, \quad \hat{c}_{1}^{\prime}=\hat{c}_{0} \hat{a}_{2}-\hat{a}_{0} \hat{c}_{2}, \quad \hat{c}_{2}^{\prime}=\hat{c}_{0} \hat{a}_{3}-\hat{a}_{0} \hat{c}_{3} .
$$


For large $|s| \gg 1$ we have immediately the following asymptotic formulae from the above:

$$
\hat{c}_{0}=\rho_{12}^{2} T(T+1), \quad \hat{c}_{1} \approx \rho_{12}^{2}(2 T+1) s, \quad \hat{c}_{2} \approx \rho_{12}^{2} s^{2}, \quad \hat{c}_{3} \approx-\rho_{23}^{2} s^{2}
$$

and

$$
\begin{aligned}
& \hat{c}_{0}^{\prime} \approx\left\{r^{2} \rho_{12}^{2}(T-1)(3 T+1)-\rho_{12}^{4} T^{2}\right\} s, \quad \hat{c}_{1}^{\prime} \approx\left\{r^{2} \rho_{12}^{2}(3 T-1)-\rho_{12}^{4} T\right\} s^{2}, \\
& \hat{c}_{2}^{\prime} \approx\left\{\left(\rho_{23}^{2}-r^{2}\right) \rho_{12}^{2} T(T+1)+r^{2} \rho_{23}^{2}(T-1)^{2}-T^{2} \rho_{23}^{2} \rho_{12}^{2}\right\} s^{2} .
\end{aligned}
$$

The resultant of $\tilde{g}_{3}^{b}$ and $\tilde{g}_{1}^{b}$ relative to $t_{1}$ is given by the Sylvester determinant as follows:

$$
R^{b}(s):=\left|\begin{array}{cccccc}
\hat{c}_{0} & \hat{c}_{1} & \hat{c}_{2} & \hat{c}_{3} & & \\
& \hat{c}_{0} & \hat{c}_{1} & \hat{c}_{2} & \hat{c}_{3} & \\
& & \hat{c}_{0} & \hat{c}_{1} & \hat{c}_{2} & \hat{c}_{3} \\
\hat{a}_{0} & \hat{a}_{1} & \hat{a}_{2} & \hat{a}_{3} & & \\
& \hat{a}_{0} & \hat{a}_{1} & \hat{a}_{2} & \hat{a}_{3} & \\
& & \hat{a}_{0} & \hat{a}_{1} & \hat{a}_{2} & \hat{a}_{3}
\end{array}\right|,
$$

which is a polynomial in $s$ of degree 8 . We notice that, from (5.22), the resultant of $\tilde{g}_{3}^{b}$ and $\tilde{g}_{13}^{b}$ relative to $t_{1}$ coincides with $R^{b}(s)$ up to a constant, which is explicitly written as

$$
\left|\begin{array}{ccccc}
\hat{c}_{0} & \hat{c}_{1} & \hat{c}_{2} & \hat{c}_{3} & \\
& \hat{c}_{0} & \hat{c}_{1} & \hat{c}_{2} & \hat{c}_{3} \\
\hat{c}_{0}^{\prime} & \hat{c}_{1}^{\prime} & \hat{c}_{2}^{\prime} & & \\
& \hat{c}_{0}^{\prime} & \hat{c}_{1}^{\prime} & \hat{c}_{2}^{\prime} & \\
& & \hat{c}_{0}^{\prime} & \hat{c}_{1}^{\prime} & \hat{c}_{2}^{\prime}
\end{array}\right| \equiv \hat{c}_{0}^{2} R^{\mathrm{b}}(s) .
$$

Owing to Lemma 5.2 we have

$$
\tilde{g}_{1}^{\mathrm{b}}(-T, 1)=\tilde{g}_{1}(1,0)=0, \quad \tilde{g}_{3}^{\mathrm{b}}(-T, 1)=\tilde{g}_{3}(1,0)=0 .
$$

Hence we have the equality $R^{b}(-T)=0$. Namely $R^{b}(s)$ can be represented by the product

$$
R^{\mathrm{b}}(s)=\gamma(s+T) \prod_{j=1}^{7}\left(s-\zeta_{j}^{\prime \prime}\right),
$$

where $\zeta_{j}^{\prime \prime}=s\left(\mathbf{c}_{j}\right)(1 \leq j \leq 7)$ and $\gamma$ denotes a constant independent of $s$.

In the same way as Lemma 5.8 we have the following.

\section{Lemma 5.13. Both}

$$
U^{\mathrm{b}}=\hat{c}_{0}\left(\hat{c}_{1}^{\prime 2}-\hat{c}_{0}^{\prime} \hat{c}_{2}^{\prime}\right)-\hat{c}_{1} \hat{c}_{0}^{\prime} \hat{c}_{1}^{\prime}+\hat{c}_{2} \hat{c}_{0}^{\prime 2} \text { and } V^{\mathrm{b}}=-\hat{c}_{0} \hat{c}_{1}^{\prime} \hat{c}_{2}^{\prime}+\hat{c}_{1} \hat{c}_{0}^{\prime} \hat{c}_{2}^{\prime}-\hat{c}_{3} \hat{c}_{0}^{\prime 2}
$$

are polynomials in s of degree four, which satisfy the equation

$$
U^{\mathrm{b}} t_{1}-V^{\mathrm{b}}=\hat{c}_{0}^{\prime 2} \tilde{g}_{3}^{\mathrm{b}}-\left(\hat{c}_{0} \hat{c}_{0}^{\prime} t_{1}+\hat{c}_{1} \hat{c}_{0}^{\prime}-\hat{c}_{0} \hat{c}_{1}^{\prime}\right) \tilde{g}_{13}^{\mathrm{b}} \equiv 0,
$$

so that, from (5.25), the following equality holds true

$$
\hat{c}_{0}^{\prime 2}\left\{\hat{c}_{0}^{2} R^{\mathrm{b}}(s)\right\}=\hat{c}_{0}^{\prime}\left\{V^{\mathrm{b}}\right\}^{2}+\hat{c}_{1}^{\prime} V^{\mathrm{b}} U^{\mathrm{b}}+\hat{c}_{2}^{\prime}\left\{U^{\mathrm{b}}\right\}^{2} .
$$


The rational curve defined by

$$
t_{1}=\omega(s), \quad \omega(s)=\frac{V^{\mathrm{b}}(s)}{U^{\mathrm{b}}(s)}
$$

is an interpolating curve of the set $\left\{\mathbf{c}_{j}\right\}$ relative to the admissible coordinates $s, t_{1}$.

Seeing that $U^{\mathrm{b}}(s) \approx O\left(s^{4}\right)$ for large $|s| \gg 1$ we have from Lemma 5.13

$$
\hat{c}_{0}^{2} \hat{c}_{0}^{\prime 2} R^{b} \approx\left(\hat{c}_{1}^{\prime} V^{b}+\hat{c}_{2}^{\prime} U^{b}\right) U^{b} \approx\left(-\hat{c}_{3} \hat{c}_{1}^{\prime}+\hat{c}_{2} \hat{c}_{2}^{\prime}\right) \hat{c}_{0}^{\prime 2} U^{b}
$$

i.e.,

$$
\hat{c}_{0}^{2} R^{\mathrm{b}} \approx\left(-\hat{c}_{3} \hat{c}_{1}^{\prime}+\hat{c}_{2} \hat{c}_{2}^{\prime}\right) U^{\mathrm{b}} .
$$

Furthermore (5.23) and (5.24) show the asymptotic formulae for large $|s| \gg 1$

$$
\begin{aligned}
\hat{c}_{0} & =\rho_{12}^{2} T(T+1), \\
-\hat{c}_{3} \hat{c}_{1}^{\prime}+\hat{c}_{2} \hat{c}_{2}^{\prime} & \approx r^{2} \rho_{12}^{2}\left(\rho_{23}^{2}-\rho_{12}^{2}\right) T(T+1) s^{4}, \\
U^{\mathrm{b}} & \approx-r^{2} \rho_{12}^{6}\left(\rho_{12}^{2}-4 r^{2}\right) T(T+1) s^{4} .
\end{aligned}
$$

(5.26) - (5.30) derive the following lemma.

Lemma 5.14. $R^{b}$ has the following expression

$$
R^{\mathrm{b}}(s)=\gamma(s+T) \prod_{j=1}^{7}\left(s-\zeta_{j}^{\prime \prime}\right),
$$

where $\gamma$ denotes the constant given by

$$
\gamma=-r^{4} \rho_{12}^{4}\left(\rho_{23}^{2}-\rho_{12}^{2}\right)\left(\rho_{12}^{2}-4 r^{2}\right)=-r^{4} \rho_{12}^{2}\left(\rho_{23}^{2}-\rho_{12}^{2}\right) B(0 \star 12) .
$$

Let us now evaluate the value $R^{b}(s)$ at $s=-1$. First notice that

$$
\begin{aligned}
\hat{a}_{0}(-1)= & \frac{r^{2}\left(\rho_{13}^{2}-\rho_{12}^{2}-\rho_{23}^{2}\right)^{2}-\left(\rho_{13}^{2}-\rho_{12}^{2}\right)^{2} \rho_{12}^{2}}{\rho_{23}^{4}}, \\
\hat{a}_{1}(-1)= & \frac{-r^{2}\left(\rho_{13}^{2}-\rho_{12}^{2}+3 \rho_{23}^{2}\right)\left(\rho_{13}^{2}-\rho_{12}^{2}-\rho_{23}^{2}\right)}{\rho_{23}^{4}} \\
& +\frac{\left(2 \rho_{12}^{2}-\rho_{13}^{2}\right) \rho_{23}^{2}+\left(\rho_{13}^{2}-\rho_{12}^{2}\right)\left(3 \rho_{12}^{2}+\rho_{13}^{2}\right)}{\rho_{23}^{2}}, \\
\hat{a}_{2}(-1)= & 4 r^{2} \frac{\rho_{13}^{2}-\rho_{12}^{2}}{\rho_{23}^{2}}-3 \rho_{13}^{2}-\rho_{12}^{2}+2 \rho_{23}^{2}, \\
\hat{a}_{3}(-1)= & \rho_{23}^{2}-4 r^{2},
\end{aligned}
$$

and

$$
\begin{aligned}
& \hat{c}_{0}(-1)=\frac{\rho_{12}^{2}\left(\rho_{13}^{2}-\rho_{12}^{2}\right)\left(\rho_{13}^{2}-\rho_{12}^{2}+\rho_{23}^{2}\right)}{\rho_{23}^{4}}, \quad \hat{c}_{1}(-1)=\frac{\left(\rho_{13}^{2}-\rho_{12}^{2}\right)\left(\rho_{23}^{2}-\rho_{12}^{2}-\rho_{13}^{2}\right)}{\rho_{23}^{2}}, \\
& \hat{c}_{2}(-1)=\hat{c}_{3}(-1)=0 .
\end{aligned}
$$


In view of (5.31) and (5.32) by definition of $R^{b}$ we have

$$
R^{\mathrm{b}}(-1)=\left\{\hat{a}_{3}(-1)\right\}^{2}\left\{\hat{c}_{0}(-1)\right\}^{3} \tilde{g}_{1}^{\mathrm{b}}\left(-1, t_{1}\right)
$$

for

$$
t_{1}=-\frac{\hat{c}_{1}(-1)}{\hat{c}_{0}(-1)}=\frac{\rho_{23}^{2}\left(\rho_{12}^{2}+\rho_{13}^{2}-\rho_{23}^{2}\right)}{\rho_{12}^{2}\left(\rho_{13}^{2}+\rho_{23}^{2}-\rho_{12}^{2}\right)} .
$$

The corresponding value of $t_{2}$ satisfies

$$
t_{2}=\frac{\rho_{13}^{2}\left(\rho_{12}^{2}+\rho_{23}^{2}-\rho_{13}^{2}\right)}{\rho_{12}^{2}\left(\rho_{13}^{2}+\rho_{23}^{2}-\rho_{12}^{2}\right)}
$$

such that $-1=-T t_{1}-t_{2}$. Hence

$$
\tilde{g}_{1}^{\mathrm{b}}\left(-1, t_{1}\right)=\tilde{g}_{1}\left(t_{1}, t_{2}\right)
$$

with $t_{1}$ and $t_{2}$ given by (5.34) and (5.35), respectively.

Seeing that

$$
B\left(\begin{array}{lll}
0 & j & l \\
0 & k & l
\end{array}\right)=\rho_{j l}^{2}+\rho_{k l}^{2}-\rho_{j k}^{2}
$$

one can prove the following lemma by a direct calculation:

Lemma 5.15. For $t_{1}, t_{2}$ being given by (5.34) and (5.35) the following identity holds:

$$
\tilde{g}_{1}\left(t_{1}, t_{2}\right)=\frac{\left(\rho_{23}^{2}-\rho_{12}^{2}\right) B\left(\begin{array}{lll}
0 & 1 & 2 \\
0 & 3 & 2
\end{array}\right) B(0123) B(0 \star 123)}{2 \rho_{12}^{6}\left\{B\left(\begin{array}{lll}
0 & 1 & 3 \\
0 & 2 & 3
\end{array}\right)\right\}^{3}} .
$$

Proof. From (5.10) $\tilde{g}_{1}$ can be written as $\tilde{g}_{1}\left(t_{1}, t_{2}\right)=r^{2} \beta_{0}+\beta_{1}+\beta_{2}$, where

$$
\beta_{0}=\left(t_{1}-1\right) t_{\infty}, \quad \beta_{1}=\left(-\rho_{2}^{2} t_{1}+\rho_{23}^{2}\right) t_{2}^{2}, \quad \beta_{2}=2\left(-\rho_{12}^{2}+\rho_{23}^{2}\right) t_{1} t_{2}+\rho_{13}^{2} t_{1}\left(t_{1}-1\right) .
$$

One sees that

$$
t_{\infty}=\frac{1}{\rho_{12}^{2} B\left(\begin{array}{lll}
0 & 1 & 3 \\
0 & 2 & 3
\end{array}\right)}\left\{\rho_{12}^{2} B\left(\begin{array}{lll}
0 & 1 & 3 \\
0 & 2 & 3
\end{array}\right)+\rho_{23}^{2} B\left(\begin{array}{lll}
0 & 2 & 1 \\
0 & 3 & 1
\end{array}\right)+\rho_{13}^{2} B\left(\begin{array}{lll}
0 & 1 & 2 \\
0 & 3 & 2
\end{array}\right)\right\}=-\frac{B\left(\begin{array}{lll}
0 & 23
\end{array}\right)}{\rho_{12}^{2} B\left(\begin{array}{lll}
0 & 1 & 3 \\
0 & 2 & 3
\end{array}\right)} .
$$

Hence

$$
\beta_{0}=-\frac{\left(\rho_{23}^{2}-\rho_{12}^{2}\right)\{B(0123)\}^{2} B\left(\begin{array}{lll}
0 & 1 & 2 \\
0 & 3 & 2
\end{array}\right)}{\rho_{12}^{6}\left\{B\left(\begin{array}{lll}
0 & 1 & 3 \\
0 & 2 & 3
\end{array}\right)\right\}^{3}} .
$$


On the other hand we have

$$
\begin{aligned}
& \beta_{1}=\frac{2 \rho_{2}^{2} \rho_{23}^{2} \rho_{13}^{4}\left(\rho_{23}^{2}-\rho_{12}^{2}\right)\left\{B\left(\begin{array}{lll}
0 & 1 & 2 \\
0 & 3 & 2
\end{array}\right)\right\}^{2}}{\rho_{12}^{6}\left\{B\left(\begin{array}{lll}
0 & 1 & 3 \\
0 & 2 & 3
\end{array}\right)\right\}^{3}}, \\
& \beta_{2}=\frac{\rho_{12}^{2} \rho_{13}^{2} \rho_{23}^{2}\left(\rho_{23}^{2}-\rho_{12}^{2}\right) B\left(\begin{array}{lll}
0 & 1 & 3 \\
0 & 2 & 3
\end{array}\right) B\left(\begin{array}{lll}
0 & 1 & 2 \\
0 & 3 & 2
\end{array}\right) B\left(\begin{array}{lll}
0 & 2 & 1 \\
0 & 3 & 1
\end{array}\right)}{\rho_{12}^{6}\left\{B\left(\begin{array}{lll}
0 & 1 & 3 \\
0 & 2 & 3
\end{array}\right)\right\}^{3}} \text {. }
\end{aligned}
$$

In view of $B(0 \star 123)=-2 r^{2} B(0123)-2 \rho_{12}^{2} \rho_{13}^{2} \rho_{23}^{2}$, one can derive the formula (5.36).

(5.33) and (5.35) imply the following lemma:

Lemma 5.16. $R^{b}(-1)$ has the product formula

$$
R^{\mathrm{b}}(-1)=\frac{\left(\rho_{13}^{2}-\rho_{12}^{2}\right)^{3}\left(\rho_{23}^{2}-\rho_{12}^{2}\right)\{B(0 \star 23)\}^{2} B(0123) B(0 \star 123)}{2 \rho_{23}^{16}} B\left(\begin{array}{lll}
0 & 1 & 2 \\
0 & 3 & 2
\end{array}\right) .
$$

Due to Lemmas 5.14 and 5.16

Corollary 5.17. The norm formula for $s+1$ is given by

$$
\mathcal{N}(s+1)=-\prod_{j=1}^{7}\left(-1-\zeta_{j}^{\prime \prime}\right)=-\frac{R^{b}(-1)}{(T-1) \gamma}=-\frac{\left(\rho_{13}^{2}-\rho_{12}^{2}\right)^{3}\{B(0 \star 23)\}^{2} B(0123) B(0 \star 123)}{2 r^{4} \rho_{12}^{2} \rho_{23}^{14} B(0 \star 12)} .
$$

From Proposition 5.11, (5.21) and Corollary 5.17

Lemma 5.18.

$$
\mathcal{N}\left(f_{2}-f_{3}\right)=\frac{1}{2 \times 3^{3}} \frac{\left(\rho_{13}^{2}-\rho_{12}^{2}\right)^{3}\{B(0 \star 23)\}^{2} B(0 \star 123)}{\rho_{12}^{2} \rho_{13}^{2} \rho_{23}^{2}} .
$$

Finally one can conclude the following.

\section{Theorem 5.19.}

$$
\begin{aligned}
& \mathcal{N}\left(f_{1}\right)=\frac{1}{2 \times 3^{4}} \frac{r^{2} B(0 \star 12) B(0 \star 13) B(0 \star 123)}{\rho_{23}^{2}}, \\
& \mathcal{N}\left(f_{2}\right)=\frac{1}{2 \times 3^{4}} \frac{r^{2} B(0 \star 12) B(0 \star 23) B(0 \star 123)}{\rho_{13}^{2}}, \\
& \mathcal{N}\left(f_{3}\right)=\frac{1}{2 \times 3^{4}} \frac{r^{2} B(0 \star 13) B(0 \star 23) B(0 \star 123)}{\rho_{12}^{2}} .
\end{aligned}
$$

Proof. We prove the formula for $\mathcal{N}\left(f_{2}\right)$. By definition

$$
\mathcal{N}\left(f_{2}\right)=\frac{\mathcal{N}\left(f_{2}-f_{3}\right)}{\mathcal{N}\left(1-t_{2}\right)}
$$

One may apply the formulae in Proposition 5.7 and Lemma 5.18, and obtains Theorem 5.19 for $\mathcal{N}\left(f_{2}\right)$. The formulae for $\mathcal{N}\left(f_{j}\right)$ for $j=1,3$ are also valid by symmetric argument. 


\section{Circle Arrangements (ii), Discriminant Formula}

In this section we want to discuss a formula related to the norm of "Hessian" of the level function $\mathfrak{R e} F$. Concerning the derivatives relative to $t_{1}$ of $\bar{\psi}\left(t_{1}\right)$ and $R\left(t_{1}\right)$, Lemma 5.4 shows

$$
\psi^{\prime}\left(t_{1}\right) \equiv \frac{c_{0}^{2}}{U^{2}} R^{\prime}\left(t_{1}\right)
$$

A direct computation gives the following:

\section{Lemma 6.1.}

$$
\frac{\partial\left(\tilde{g}_{2}, \tilde{g}_{3}\right)}{\partial\left(t_{1}, t_{2}\right)} \equiv-r^{2} \frac{B(0 \star 12) \rho_{12}^{2}\left(\rho_{12}^{2}-\rho_{13}^{2}\right)}{U} t_{1} \bar{\psi}^{\prime}\left(t_{1}\right)
$$

Proof. Partial derivation of (5.13) with respect to $t_{2}$ gives the identity

$$
U\left(t_{1}\right)=\frac{\partial \tilde{g}_{23}\left(t_{1}, t_{2}\right)}{\partial t_{2}} .
$$

On the other hand, from Lemma 5.4 we have $\tilde{g}_{23}\left(t_{1}, \omega\left(t_{1}\right)\right)=0$. By derivation relative to $t_{1}$

$$
\frac{\partial \tilde{g}_{23}\left(t_{1}, \omega\left(t_{1}\right)\right)}{\partial t_{1}}+\frac{\partial \tilde{g}_{23}\left(t_{1}, \omega\left(t_{1}\right)\right)}{\partial t_{2}} \omega^{\prime}\left(t_{1}\right)=0
$$

In the same way by derivation of $\psi\left(t_{1}\right)$ relative to $t_{1}$ (see Lemma 5.4)

$$
\psi^{\prime}\left(t_{1}\right)=\frac{\partial \tilde{g}_{3}\left(t_{1}, \omega\left(t_{1}\right)\right)}{\partial t_{1}}+\frac{\partial \tilde{g}_{3}\left(t_{1}, \omega\left(t_{1}\right)\right)}{\partial t_{2}} \omega^{\prime}\left(t_{1}\right)
$$

From (5.13)

(6.1) implies

$$
\psi^{\prime}\left(t_{1}\right)=\frac{\partial\left(\tilde{g}_{3}, \tilde{g}_{23}\right)}{\partial\left(t_{1}, t_{2}\right)} / \frac{\partial \tilde{g}_{23}}{\partial t_{2}}=-\frac{c_{0}^{2}}{U} \frac{\partial\left(\tilde{g}_{2}, \tilde{g}_{3}\right)}{\partial\left(t_{1}, t_{2}\right)}
$$

which completes Lemma 6.1.

$$
R^{\prime}\left(t_{1}\right) \equiv-U\left(t_{1}\right) \frac{\partial\left(\tilde{g}_{2}, \tilde{g}_{3}\right)}{\partial\left(t_{1}, t_{2}\right)}
$$

Lemma 6.2. The identity holds

$$
d G_{1} \wedge d G_{2} \equiv-\frac{t_{1} t_{2}}{1-t_{2}} \frac{L_{12}^{4}}{f_{3}^{4}\left(\alpha_{21} \alpha_{12}\right)^{3}} d \tilde{g}_{2} \wedge d \tilde{g}_{3} .
$$

Proof. Put $G_{13}=x_{2} G_{1}-\left(x_{1}+\alpha_{21}\right) G_{2}$ and $G_{23}=\left(x_{2}+\alpha_{12}\right) G_{1}-\left(x_{1}+\alpha_{11}\right) G_{2}$, then

$$
d G_{13} \wedge d G_{23} \equiv L_{12} d G_{1} \wedge d G_{2} \text {. }
$$

Further it holds

$$
g_{2}=f_{2} f_{3} G_{23}, \quad g_{3}=L_{12} f_{3}^{2}\left(\frac{1-t_{2}}{t_{1}} G_{13}-\frac{1-t_{1}}{t_{2}} G_{23}\right),
$$

so that

$$
d g_{2} \wedge d g_{3} \equiv-\frac{1-t_{2}}{t_{1} t_{2}} f_{3}^{4} L_{12} d G_{13} \wedge d G_{23}
$$

From (5.5) and (5.9) we obtain

$$
d g_{2} \wedge d g_{3} \equiv \frac{\left(\alpha_{21} \alpha_{12}\right)^{3}}{t_{\infty}^{6}} d \tilde{g}_{2} \wedge d \tilde{g}_{3}
$$

where $4 \alpha_{21}^{2} \alpha_{12}^{2}=-B(0123)$. Summing up these equalities of Jacobian implies Lemma 6.2. 
By definition we have

$$
\operatorname{Hess}(F)=\frac{\partial\left(G_{1}, G_{2}\right)}{\partial\left(x_{1}, x_{2}\right)}, \quad \frac{\partial\left(x_{1}, x_{2}\right)}{\partial\left(t_{1}, t_{2}\right)}=\frac{\sqrt{-B(0123)}}{2 t_{\infty}^{3}} .
$$

By using these equalities one can prove the following:

Proposition 6.3. Suppose that $\rho_{12}^{2} \neq \rho_{13}^{2}$. Then, at each critical point $\mathbf{c}_{j}(1 \leq j \leq 7)$ the Hessian of $F$ at $\mathbf{c}_{j}$ is expressed as

$$
[\operatorname{Hess}(F)]_{\mathbf{c}_{j}}=-\left[\frac{t_{1} t_{2}}{\left(1-t_{2}\right) t_{\infty} U\left(t_{1}\right)} \frac{R^{\prime}\left(t_{1}\right)}{f_{3}(x)^{4}}\right]_{\mathbf{c}_{j}},
$$

such that $\zeta_{j}=\left[t_{1}\right]_{\mathbf{c}_{j}}$ and $t_{2}=V\left(t_{1}\right) / U\left(t_{1}\right)$.

Proof. Indeed (5.6), (5.7) and Lemma 6.2 mean

$$
\begin{aligned}
\operatorname{Hess}(F) & =\frac{\partial\left(G_{1}, G_{2}\right)}{\partial\left(x_{1}, x_{2}\right)}=\frac{\partial\left(G_{1}, G_{2}\right)}{\partial\left(t_{1}, t_{2}\right)} / \frac{\partial\left(x_{1}, x_{2}\right)}{\partial\left(t_{1}, t_{2}\right)}=\frac{t_{1} t_{2} L_{12}^{4}}{\left(1-t_{2}\right) f_{3}^{4}\left(\alpha_{21} \alpha_{12}\right)^{3}} \frac{t_{\infty}^{3}}{\alpha_{21} \alpha_{12}} \frac{\partial\left(\tilde{g}_{2}, \tilde{g}_{3}\right)}{\partial\left(t_{1}, t_{2}\right)} \\
& =-\frac{t_{1} t_{2}}{\left(1-t_{2}\right) t_{\infty} U\left(t_{1}\right)} \frac{R^{\prime}\left(t_{1}\right)}{f_{3}(x)^{4}},
\end{aligned}
$$

which completes the proof.

We now assume the condition $\left(C_{3}\right)$ : (i) $\rho_{12}^{2} \neq \rho_{23}^{2}$, and (ii) the set $\left\{\zeta_{j} \mid 1 \leq j \leq 7\right\}$ consists of seven different complex numbers and the projection mapping to the $t_{1}$-coordinate

$$
t_{1}: \mathbf{c}_{j} \longmapsto \zeta_{j}=t_{1}\left(\mathbf{c}_{j}\right) \quad(1 \leq j \leq 7)
$$

is bijective.

Then the following holds.

Lemma 6.4. $\mathcal{N}(U) \neq 0$.

Proof. Suppose on the contrary $\mathcal{N}(U)=0$. There exists $\mathbf{c}_{j}(1 \leq j \leq 7)$ such that $U\left(\zeta_{j}\right)=0$ for $\zeta_{j}=t_{1}\left(\mathbf{c}_{j}\right)$. Since by definition $R\left(\zeta_{j}\right)=0$, Lemma 5.4 shows

$$
c_{0}\left(\zeta_{j}\right)^{2} R\left(\zeta_{j}\right)=c_{0}\left(\zeta_{j}\right) V\left(\zeta_{j}\right)^{2}=0 .
$$

First assume $c_{0}\left(\zeta_{j}\right) \neq 0$. Then we also have $V\left(\zeta_{j}\right)=0$. Hence from (5.13)

$$
c_{0}\left(\zeta_{j}\right)^{2} \tilde{g}_{2}\left(\zeta_{j}, t_{2}\right)-\left(b_{0}\left(\zeta_{j}\right) c_{0}\left(\zeta_{j}\right) t_{2}+b_{1}\left(\zeta_{j}\right) c_{0}\left(\zeta_{j}\right)-b_{1}\left(\zeta_{j}\right) c_{1}\left(\zeta_{j}\right)\right) \tilde{g}_{3}\left(\zeta_{j}, t_{2}\right)=0
$$

as a polynomial in $t_{2}$. This means $\tilde{g}_{2}\left(\zeta_{j}, t_{2}\right)$ is divisible by $\tilde{g}_{3}\left(\zeta_{j}, t_{2}\right)$. Let $\xi_{1}, \xi_{2}$ be two different roots of the equation $\tilde{g}_{3}\left(\zeta_{j}, t_{2}\right)=0$. Hence there exist two different critical points $\mathbf{c}_{j}, \mathbf{c}_{k}$ such that $t_{1}\left(\mathbf{c}_{j}\right)=t_{1}\left(\mathbf{c}_{k}\right)=\zeta_{j}$ and $t_{2}\left(\mathbf{c}_{j}\right)=\xi_{1}, t_{2}\left(\mathbf{c}_{k}\right)=\xi_{2}$. This contradicts the assumption (ii) of $\left(C_{3}\right)$. Assume now $c_{0}\left(\zeta_{j}\right)=0$. Then by the definition of $U\left(t_{1}\right)$ in Lemma 5.4

$$
U\left(\zeta_{j}\right)=b_{0}\left(\zeta_{j}\right) c_{1}\left(\zeta_{j}\right)^{2}=0 .
$$

Hence $c_{1}\left(\zeta_{j}\right)=0$. This means $\zeta_{j}$ must be equal to 1 or 0 . The case $\zeta_{j}=0$ is impossible. Suppose $\zeta_{j}=1$. Then from (5.11) we have $-\rho_{12}^{2}+\rho_{23}^{2}=c_{1}(1)=0$. This fact contradicts the assumption (i) of $\left(C_{3}\right)$. Lemma 6.4 has been proved. 
Summing up Propositions 5.7, 5.11, Corollary 5.12, Theorem 5.19 and Proposition 6.3 we have

Theorem 6.5. Under the conditions $\left(C_{2}\right)$ and $\left(C_{3}\right)$ the following equality holds.

$$
\mathcal{N}(\operatorname{Hess}(F))=-C^{7} \frac{\mathcal{N}\left(t_{1}^{2} t_{2}\right)}{\mathcal{N}\left(W^{*}\right) \mathcal{N}\left(t_{\infty}\right) \mathcal{N}\left(f_{3}^{4}\right)} \text { Discr }
$$

for $W^{*}=\frac{U-V}{\rho_{13}^{2}-\rho_{12}^{2}}$, where Discr denotes the discriminant of $\bar{\psi}\left(t_{1}\right)$ relative to the basic parameter $t_{1}$

$$
\text { Discr }:=\prod_{1 \leq j<k \leq 7}\left(\zeta_{j}-\zeta_{k}\right)^{2}=-\prod_{j=1}^{7}\left[\bar{\psi}^{\prime}\left(t_{1}\right)\right]_{\zeta_{j}}
$$

and $C$ denotes the constant given by

$$
C=r^{2} \rho_{12}^{2}\left(\rho_{13}^{2}-\rho_{12}^{2}\right) B(0 \star 12) .
$$

Proof. From the identity in Lemma 5.6 the derivative of $R\left(t_{1}\right)$ with respect to $t_{1}$ has

$$
R^{\prime}\left(t_{1}\right) \equiv-r^{2} \rho_{12}^{2}\left(\rho_{13}^{2}-\rho_{12}^{2}\right) B(0 \star 12) t_{1} \bar{\psi}^{\prime}\left(t_{1}\right) .
$$

By Proposition 6.3 we have

$$
\operatorname{Hess}(F) \equiv \frac{\rho_{12}^{2} r^{2} B(0 \star 12) t_{1}^{2} t_{2} \bar{\psi}^{\prime}\left(t_{1}\right)}{W^{*} t_{\infty} f_{3}^{4}},
$$

which implies Theorem 6.5.

Remark. It is not certain if $\mathcal{N}(U) \neq 0$ holds under the weaker conditions $\left(C_{2}\right)$ and (i) of $\left(C_{3}\right)$.

\section{Circle Arrangements (iii), Case of Isosceles Triangle}

The case when $\triangle O_{1} O_{2} O_{3}$ is an isosceles triangle is an exceptional one. This section is devoted to its explanation in more detail.

Generally we may put by (5.15) and Lemma 5.6

$$
U-V=\left(\rho_{13}^{2}-\rho_{12}^{2}\right) W^{*} \quad \text { and } \quad R=\left(\rho_{13}^{2}-\rho_{12}^{2}\right) R^{*},
$$

respectively. According to (5.11)

$$
c_{0}=\rho_{12}^{2} t_{1}-\rho_{23}^{2}, \quad c_{1}=-\rho_{12}^{2} t_{1}^{2}+\rho_{23}^{2}, \quad c_{2}=\rho_{13}^{2} t_{1}\left(t_{1}-1\right),
$$

so that $c_{0}+c_{1}+c_{2}=\left(\rho_{13}^{2}-\rho_{12}^{2}\right) t_{1}\left(t_{1}-1\right)$. Using this, (5.14) is rewritten as the following identity for the polynomials $R^{*}$ and $W^{*}$

$$
c_{0}^{2} R^{*}=\left(\rho_{13}^{2}-\rho_{12}^{2}\right) c_{2}\left\{W^{*}\right\}^{2}+V\left\{\left(t_{1}^{2}-t_{1}\right) V+\left(c_{1}+2 c_{2}\right) W^{*}\right\} .
$$

Throughout this section we suppose that the equality

$$
\rho_{12}^{2}=\rho_{13}^{2}
$$


holds. Then $c_{0}+c_{1}+c_{2}=0$, and both $U-V$ and $R$ vanish identically since they are divisible by $\rho_{13}^{2}-\rho_{12}^{2}$ (see (5.15) and Lemma 5.6). We have $U=V$, so that the identity (7.2) is written as

$$
c_{0}^{2} R^{*}=U\left\{\left(t_{1}^{2}-t_{1}\right) U+\left(c_{1}+2 c_{2}\right) W^{*}\right\} .
$$

Let $\tilde{g}_{2}^{*}$ and $\tilde{g}_{3}^{*}$ be polynomials specified by

$$
\tilde{g}_{2}=\left(t_{2}-1\right) \tilde{g}_{2}^{*} \quad \text { and } \quad \tilde{g}_{3}=\left(t_{2}-1\right) \tilde{g}_{3}^{*},
$$

respectively. By the definition (5.10) of $\tilde{g}_{2}$ and $\tilde{g}_{3}$ with $\rho_{12}^{2}=\rho_{13}^{2}$, the polynomials $\tilde{g}_{2}^{*}$ and $\tilde{g}_{3}^{*}$ are written as

$$
\tilde{g}_{2}^{*}=b_{0}^{*} t_{2}^{2}+b_{1}^{*} t_{2}+b_{2}^{*} \quad \text { and } \quad \tilde{g}_{3}^{*}=c_{0}^{*} t_{2}+c_{1}^{*},
$$

where (5.11) gives the explicit forms of the coefficients

$$
\begin{gathered}
b_{0}^{*}=r^{2}, \quad b_{1}^{*}=2 r^{2} t_{1}+\rho_{23}^{2}+2 r^{2}, \quad b_{2}^{*}=\left(r^{2}-\rho_{12}^{2}\right) t_{1}^{2}+2 r^{2} t_{1}+r^{2}, \\
c_{0}^{*}=\rho_{12}^{2} t_{1}-\rho_{23}^{2}, \quad c_{1}^{*}=-\rho_{12}^{2} t_{1}\left(t_{1}-1\right) .
\end{gathered}
$$

In particular, using the chain rule $\partial \tilde{g}_{3} / \partial t_{2}=\left(t_{2}-1\right) \partial \tilde{g}_{3}^{*} / \partial t_{2}+\tilde{g}_{3}^{*}$ and $c_{0}+c_{1}+c_{2}=0$, we have

$$
c_{0}^{*}+c_{1}^{*}=\tilde{g}_{3}^{*}\left(t_{1}, 1\right)=\frac{\partial \tilde{g}_{3}}{\partial t_{2}}\left(t_{1}, 1\right)=2 c_{0}+c_{1}=-\left(c_{1}+2 c_{2}\right),
$$

namely $c_{1}+2 c_{2}=-\left(c_{0}^{*}+c_{1}^{*}\right)=\rho_{12}^{2} t_{1}\left(t_{1}-2\right)+\rho_{23}^{2}$. Since we have $U=V$, using $\tilde{g}_{2}$ and $\tilde{g}_{3}$ the identity (5.13) is equivalent to

$$
U=c_{0}^{2} \tilde{g}_{2}^{*}-\left(b_{0} c_{0} t_{2}+b_{1} c_{0}-b_{0} c_{1}\right) \tilde{g}_{3}^{*},
$$

which is independent of $t_{2}$. On the other hand, from Lemma 5.4 the polynomial $U=U\left(t_{1}\right)$ of degree 4 can be written using the monic polynomial $\bar{\psi}_{2}\left(t_{1}\right)$ such that

$$
U\left(t_{1}\right)=u_{0} t_{1}^{4}+u_{2} t_{1}^{3}+u_{3} t_{1}^{2}+u_{2} t_{1}+u_{4}=-\rho_{12}^{4}\left(\rho_{12}^{2}-4 r^{2}\right) \bar{\psi}_{2}\left(t_{1}\right),
$$

where

$$
\begin{aligned}
& u_{0}=-\rho_{12}^{4}\left(\rho_{12}^{2}-4 r^{2}\right), \quad u_{1}=\rho_{12}^{2} \rho_{23}^{2}\left(3 \rho_{12}^{2}-4 r^{2}\right), \\
& u_{2}=\rho_{23}^{2}\left\{-\rho_{12}^{2}\left(2 \rho_{23}^{2}+\rho_{12}^{2}\right)+\left(-4 \rho_{12}^{2}+\rho_{23}^{2}\right) r^{2}\right\} \\
& u_{3}=\rho_{23}^{4}\left(\rho_{12}^{2}+2 r^{2}\right), \quad u_{4}=\rho_{23}^{4} r^{2}
\end{aligned}
$$

and $\bar{\psi}_{2}\left(t_{1}\right)$ has 4 roots denoted by $\zeta_{4}, \zeta_{5}, \zeta_{6}, \zeta_{7}$. The identity stated in Proposition 6.3 is still valid by taking the limit as $\rho_{13}^{2} \rightarrow \rho_{12}^{2}$ :

$$
[\operatorname{Hess}(F)]_{\mathbf{c}_{j}}=\lim _{\rho_{13}^{2} \rightarrow \rho_{12}^{2}}\left[\frac{t_{1} t_{2} R^{\prime}}{(U-V) t_{\infty} f_{3}^{4}}\right]_{\mathbf{c}_{j}}=\left[\frac{t_{1} t_{2} R^{* \prime}}{t_{\infty} f_{3}^{4} W^{*}}\right]_{\mathbf{c}_{j}} \quad(1 \leq j \leq 7) .
$$

We denote by $\left(\zeta_{j}, \zeta_{j}^{*}\right)$ the $\left(t_{1}, t_{2}\right)$-coordinates of the critical points $\mathbf{c}_{j}(1 \leq j \leq 7)$. The critical points are divided into two parts. Three of them corresponding to $t_{1}=\zeta_{1}, \zeta_{2}, \zeta_{3}$ are contained in the line $L_{13}(x)-L_{12}(x)=0$, which is the midline of the triangle $\triangle O_{1} O_{2} O_{3}$ defined by $t_{2}=1$, while the remaining ones corresponds to $t_{1}=\zeta_{4}, \zeta_{5}, \zeta_{6}, \zeta_{7}$ for $t_{2} \neq 1$ lie outside the midline. 


\subsection{The case $t_{2} \neq 1$}

Suppose first that $t_{2} \neq 1$. Then (5.8) is equivalent to

$$
\tilde{g}_{2}^{*}=\tilde{g}_{3}^{*}=0 \text {. }
$$

The equation $\tilde{g}_{3}^{*}\left(t_{1}, t_{2}\right)=0$ can be uniquely solved:

$$
t_{2}=\frac{V^{*}}{U^{*}},
$$

where

$$
U^{*}=\frac{\partial \tilde{g}_{3}^{*}}{\partial t_{2}}=c_{0}^{*}=c_{0}=\rho_{12}^{2} t_{1}-\rho_{23}^{2}, \quad V^{*}=-c_{1}^{*}=\rho_{12}^{2} t_{1}\left(t_{1}-1\right) .
$$

Then the equation $\tilde{g}_{2}^{*}\left(t_{1}, \frac{V^{*}}{U^{*}}\right)=0$ relative to the unknown $t_{1}$ is equivalent to

$$
U\left(t_{1}\right)=b_{0}^{*}\left(V^{*}\right)^{2}+b_{1}^{*} V^{*} U^{*}+b_{2}^{*}\left(U^{*}\right)^{2}=0 .
$$

As we saw in (7.5) one may put $U\left(t_{1}\right)=\rho_{12}^{4}\left(4 r^{2}-\rho_{12}^{2}\right) \bar{\psi}_{2}\left(t_{1}\right)$ with the monic polynomial $\bar{\psi}_{2}\left(t_{1}\right)=$ $\prod_{j=4}^{7}\left(t_{1}-\zeta_{j}\right)$. The critical points $\mathbf{c}_{j}(4 \leq j \leq 7)$ are represented by the $\left(t_{1}, t_{2}\right)$-coordinates $\left(\zeta_{j}, \zeta_{j}^{*}\right)$ for $\zeta_{j}^{*}=V^{*}\left(\zeta_{j}\right) / U^{*}\left(\zeta_{j}\right)$.

Lemma 7.1. We have the following product formulae:

$$
\begin{aligned}
U(1) & =\rho_{12}^{4}\left(4 r^{2}-\rho_{12}^{2}\right) \prod_{j=4}^{7}\left(1-\zeta_{j}\right)=-\left(\rho_{23}^{2}-\rho_{12}^{2}\right)^{2}\left(\rho_{12}^{2}-4 r^{2}\right), \\
U(0) & =\rho_{12}^{4}\left(4 r^{2}-\rho_{12}^{2}\right) \prod_{j=4}^{7} \zeta_{j}=r^{2} \rho_{23}^{4}, \\
U\left(\frac{\rho_{23}^{2}}{\rho_{12}^{2}}\right) & =\rho_{12}^{4}\left(4 r^{2}-\rho_{12}^{2}\right) \prod_{j=4}^{7}\left(\frac{\rho_{23}^{2}}{\rho_{12}^{2}}-\zeta_{j}\right)=r^{2} \frac{\rho_{23}^{4}\left(\rho_{23}^{2}-\rho_{12}^{2}\right)^{2}}{\rho_{12}^{4}} \\
W^{*}\left(\frac{\rho_{23}^{2}}{\rho_{12}^{2}}\right) & =-\frac{r^{2} \rho_{23}^{2}\left(\rho_{23}^{2}-\rho_{13}^{2}\right)^{2}}{\rho_{12}^{4}}
\end{aligned}
$$

Proof. Indeed the first two identities follow from (7.5) for $\rho_{13}^{2}=\rho_{12}^{2}$. Since $U^{*}\left(\frac{\rho_{23}^{2}}{\rho_{12}^{2}}\right)=0$, (7.9) means

$$
U\left(\frac{\rho_{23}^{2}}{\rho_{12}^{2}}\right)=r^{2}\left\{V^{*}\left(\frac{\rho_{23}^{2}}{\rho_{12}^{2}}\right)\right\}^{2},
$$

which implies the third line of Lemma 7.1. The last one derives from a direct calculation.

Denote by $\bar{\psi}_{2}^{*}\left(t_{2}\right)$ the monic polynomial in $t_{2}$ related to the resultant $R_{t_{1}}\left(\tilde{g}_{2}^{*}\left(t_{1}, t_{2}\right),-\tilde{g}_{3}^{*}\left(t_{1}, t_{2}\right)\right)$ of $\tilde{g}_{2}^{*}\left(t_{1}, t_{2}\right)$ and $-\tilde{g}_{3}^{*}\left(t_{1}, t_{2}\right)$ with respect to the variable $t_{1}$ such that

$$
R_{t_{1}}\left(\tilde{g}_{2}^{*}\left(t_{1}, t_{2}\right),-\tilde{g}_{3}^{*}\left(t_{1}, t_{2}\right)\right)=r^{2}\left(4 r^{2}-\rho_{12}^{2}\right) \rho_{12}^{4} \bar{\psi}_{2}^{*}\left(t_{2}\right),
$$


where

$$
\begin{aligned}
& R_{t_{1}}\left(\tilde{g}_{2}^{*}\left(t_{1}, t_{2}\right),-\tilde{g}_{3}^{*}\left(t_{1}, t_{2}\right)\right) \\
& \quad=\left|\begin{array}{cccc}
r^{2}-\rho_{12}^{2} & 2 r^{2}\left(t_{2}+1\right) & r^{2} t_{2}^{2}+\left(\rho_{23}^{2}+2 r^{2}\right) t_{2}+r^{2} & \\
& r^{2}-\rho_{12}^{2} & 2 r^{2}\left(t_{2}+1\right) & r^{2} t_{2}^{2}+\left(\rho_{23}^{2}+2 r^{2}\right) t_{2}+r^{2} \\
\rho_{12}^{2} & -\rho_{12}^{2}\left(t_{2}+1\right) & \rho_{23}^{2} t_{2} & \rho_{23}^{2} t_{2} \\
\rho_{12}^{2} & -\rho_{12}^{2}\left(t_{2}+1\right) &
\end{array}\right| .
\end{aligned}
$$

Then $\bar{\psi}_{2}^{*}\left(t_{2}\right)$ has the roots $\zeta_{j}^{*}(4 \leq j \leq 7)$, i.e.,

$$
\bar{\psi}_{2}^{*}\left(t_{2}\right)=\prod_{j=4}^{7}\left(t_{2}-\zeta_{j}^{*}\right), \text { especially } \quad \bar{\psi}_{2}^{*}(1)=\prod_{j=4}^{7}\left(1-\zeta_{j}^{*}\right) .
$$

$\bar{\psi}_{2}^{*}\left(t_{2}\right)$ has the symmetry $t_{2}^{-2} \bar{\psi}_{2}^{*}\left(t_{2}\right)=t_{2}^{2} \bar{\psi}_{2}^{*}\left(t_{2}^{-1}\right)$. Thus, without loss of generality, we may assume

$$
\zeta_{4}^{*}=\zeta_{6}^{*-1} \text { and } \zeta_{5}^{*}=\zeta_{7}^{*-1} .
$$

By definition $\left(t_{1}, t_{2}\right)=\left(\zeta_{j}, \zeta_{j}^{*}\right)$ of the critical points $\mathbf{c}_{j}(4 \leq j \leq 7)$ satisfy the simultaneous equations

$$
U\left(t_{1}\right)=u_{0} t_{1}^{4}+u_{2} t_{1}^{3}+u_{3} t_{1}^{2}+u_{2} t_{1}+u_{4}=0 \quad \text { and } \quad t_{2}=\frac{V^{*}\left(t_{1}\right)}{U^{*}\left(t_{1}\right)},
$$

where the polynomials $U\left(t_{1}\right)$ and $V^{*}\left(t_{1}\right), U^{*}\left(t_{1}\right)$ are given by (7.5) and (7.8), respectively. This means that, for given $t_{1}=\zeta_{j}$ satisfying $U\left(t_{1}\right)=0$, we can define $t_{2}=\zeta_{j}^{*}$ by $t_{2}=V^{*}\left(t_{1}\right) / U^{*}\left(t_{1}\right)$. Conversely, for given $t_{2}=\zeta_{j}^{*}$ we can fix $t_{1}=\zeta_{j}$ uniquely as follows:

Lemma 7.2. For an arbitrary $t_{2} \in \mathbb{C}$ there exists a unique solution $t_{1} \in \mathbb{C}$ of the simultaneous equations (7.14), which is expressed as

$$
t_{1} \equiv-\frac{\hat{u}_{1}\left(t_{2}\right)}{\hat{u}_{0}\left(t_{2}\right)}
$$

where $\hat{u}_{1}\left(t_{2}\right)$ and $\hat{u}_{0}\left(t_{2}\right)$ are the polynomials in $t_{2}$ of degree 3 given by

$$
\begin{aligned}
\rho_{12}^{6} \hat{u}_{1}\left(t_{2}\right) & =\left|\begin{array}{cccc}
-u_{0} & \rho_{12}^{2} & 0 & 0 \\
-u_{1} & -\rho_{12}^{2}\left(1+t_{2}\right) & \rho_{12}^{2} & 0 \\
-u_{2} & \rho_{23}^{2} t_{2} & -\rho_{12}^{2}\left(1+t_{2}\right) & \rho_{12}^{2} \\
-u_{4} & 0 & 0 & \rho_{23}^{2} t_{2}
\end{array}\right|, \\
\rho_{12}^{6} \hat{u}_{0}\left(t_{2}\right) & =\left|\begin{array}{cccc}
-u_{0} & \rho_{12}^{2} & 0 & 0 \\
-u_{1} & -\rho_{12}^{2}\left(1+t_{2}\right) & \rho_{12}^{2} & 0 \\
-u_{2} & \rho_{23}^{2} t_{2} & -\rho_{12}^{2}\left(1+t_{2}\right) & \rho_{12}^{2} \\
-u_{3} & 0 & \rho_{23}^{2} t_{2} & -\rho_{12}^{2}\left(1+t_{2}\right)
\end{array}\right| .
\end{aligned}
$$

Proof. The simultaneous equations (7.14) are equivalent to $U\left(t_{1}\right)=0$ and $V^{*}\left(t_{1}\right)-U^{*}\left(t_{1}\right) t_{2}=0$. The latter equation is written as $\hat{v}_{0} t_{1}^{2}+\hat{v}_{1} t_{1}+\hat{v}_{2}=0$, where $\hat{v}_{0}=\rho_{12}^{2}, \hat{v}_{1}=\rho_{12}^{2}\left(1+t_{2}\right), \hat{v}_{2}=\rho_{23}^{2} t_{2}$. For given $t_{2}$ if we can fix $\hat{w}_{0}, \hat{w}_{1}, \hat{w}_{2}$ and $\hat{u}_{0}, \hat{u}_{1}$ such that

$$
\left(\hat{v}_{0} t_{1}^{2}+\hat{v}_{1} t_{1}+\hat{v}_{2}\right)\left(\hat{w}_{0} t_{1}^{2}+\hat{w}_{1} t_{1}+\hat{w}_{2}\right)+\hat{u}_{0} t_{1}+\hat{u}_{1}-\left(u_{0} t_{1}^{4}+u_{2} t_{1}^{3}+u_{3} t_{1}^{2}+u_{2} t_{1}+u_{4}\right)=0,
$$


then $t_{1}=-\hat{u}_{1} / \hat{u}_{0}$ is the solution. Comparing the coefficients of $t_{1}^{i}(0 \leq i \leq 4)$ on both sides, we immediately have the following relations:

$$
\left(\begin{array}{c}
0 \\
0 \\
0 \\
-\hat{u}_{0}
\end{array}\right)=\left(\begin{array}{cccc}
-u_{0} & \hat{v}_{0} & 0 & 0 \\
-u_{1} & \hat{v}_{1} & \hat{v}_{0} & 0 \\
-u_{2} & \hat{v}_{2} & \hat{v}_{1} & \hat{v}_{0} \\
-u_{3} & 0 & \hat{v}_{2} & \hat{v}_{1}
\end{array}\right)\left(\begin{array}{c}
1 \\
\hat{w}_{0} \\
\hat{w}_{1} \\
\hat{w}_{2}
\end{array}\right), \quad\left(\begin{array}{c}
0 \\
0 \\
0 \\
-\hat{u}_{1}
\end{array}\right)=\left(\begin{array}{cccc}
-u_{0} & \hat{v}_{0} & 0 & 0 \\
-u_{1} & \hat{v}_{1} & \hat{v}_{0} & 0 \\
-u_{2} & \hat{v}_{2} & \hat{v}_{1} & \hat{v}_{0} \\
-u_{4} & 0 & 0 & \hat{v}_{2}
\end{array}\right)\left(\begin{array}{c}
1 \\
\hat{w}_{0} \\
\hat{w}_{1} \\
\hat{w}_{2}
\end{array}\right) .
$$

Using the Laplace expansion for the above relations, we obtain (7.17) and (7.16), respectively.

For the coordinates $\left(\zeta_{j}, \zeta_{j}^{*}\right)$ of the critical point $\mathbf{c}_{j}(4 \leq j \leq 7)$ Lemma 7.2 indicates the one-to-one correspondence between $\zeta_{j}$ and $\zeta_{j}^{*}$ under $U^{*}\left(\zeta_{j}\right) \neq 0$ and $\hat{u}_{0}\left(\zeta_{j}^{*}\right) \neq 0$. In particular,

Corollary 7.3. For $t_{1}$ specified by (7.15) as a solution of (7.14), the continuous limit of $t_{1}$ as $t_{2} \rightarrow 1$ is given by

$$
t_{1} \rightarrow-\frac{\hat{u}_{1}(1)}{\hat{u}_{0}(1)} \quad\left(t_{2} \rightarrow 1\right),
$$

where $\hat{u}_{0}(1)$ and $\hat{u}_{1}(1)$ are explicitly written as

$$
\begin{aligned}
& \hat{u}_{1}(1)=\rho_{12}^{-2} \rho_{23}^{2}\left(\rho_{12}^{2}-\rho_{23}^{2}\right)\left\{r^{2}\left(\rho_{23}^{2}-16 \rho_{12}^{2}\right)+2 \rho_{12}^{2}\left(2 \rho_{12}^{2}-\rho_{23}^{2}\right)\right\}, \\
& \hat{u}_{0}(1)=2\left(\rho_{12}^{2}-\rho_{23}^{2}\right)\left\{4 r^{2}\left(4 \rho_{12}^{2}-\rho_{23}^{2}\right)-\rho_{12}^{2}\left(4 \rho_{12}^{2}-3 \rho_{23}^{2}\right)\right\},
\end{aligned}
$$

under $\hat{u}_{0}(1) \neq 0$.

\subsection{The case $t_{2}=1$}

Suppose second $t_{2}=1$. Then $\tilde{g}_{2}=\tilde{g}_{3}=0$ automatically. From $(5.8), \tilde{g}_{1}\left(t_{1}, 1\right)=0$. According to (5.12) with the condition $\rho_{13}^{2}=\rho_{12}^{2}$, we may put the monic polynomial $\bar{\psi}_{1}\left(t_{1}\right)$ as

$$
r^{2} \bar{\psi}_{1}\left(t_{1}\right):=\tilde{g}_{1}\left(t_{1}, 1\right)=r^{2} t_{1}^{3}+\left(\rho_{12}^{2}+3 r^{2}\right) t_{1}^{2}+2\left(\rho_{23}^{2}-2 \rho_{12}^{2}\right) t_{1}+\rho_{23}^{2}-4 r^{2}
$$

and denote the roots of the equation

$$
\bar{\psi}_{1}\left(t_{1}\right)=0
$$

by $\zeta_{1}, \zeta_{2}, \zeta_{3}$. For $1 \leq j \leq 3$ the point $\mathbf{c}_{j}$ corresponds to the $\left(t_{1}, t_{2}\right)$-coordinates $\left(\zeta_{j}, 1\right)$. One has by definition and from (7.10) of Lemma 7.1:

$$
\begin{aligned}
& r^{2} \prod_{j=1}^{3}\left(1-\zeta_{j}\right)=r^{2} \bar{\psi}_{1}(1)=3\left(\rho_{23}^{2}-\rho_{12}^{2}\right) \\
& \prod_{j=4}^{7} c_{0}\left(\zeta_{j}\right)=\rho_{12}^{8} \bar{\psi}_{2}\left(\frac{\rho_{23}^{2}}{\rho_{12}^{2}}\right)=\frac{\rho_{12}^{8}}{\rho_{12}^{4}\left(4 r^{2}-\rho_{12}^{2}\right)} U\left(\frac{\rho_{23}^{2}}{\rho_{12}^{2}}\right)=\frac{r^{2} \rho_{23}^{4}\left(\rho_{23}^{2}-\rho_{12}^{2}\right)^{2}}{4 r^{2}-\rho_{12}^{2}} .
\end{aligned}
$$

We consider the resultant $R_{t_{1}}\left(\tilde{g}_{1}\left(t_{1}, t_{2}\right),-\tilde{g}_{3}^{*}\left(t_{1}, t_{2}\right)\right)$ at $t_{2}=1$. Then one can show by a direct calculation the following connection between the resultant of $\tilde{g}_{1}\left(t_{1}, 1\right),-\tilde{g}_{3}^{*}\left(t_{1}, 1\right)=c_{1}+2 c_{2}$ and the value $\bar{\psi}_{2}^{*}(1)$ : 
Lemma 7.4. The resultant of the two polynomials

$$
\tilde{g}_{1}\left(t_{1}, 1\right)=r^{2} \bar{\psi}_{1}\left(t_{1}\right) \quad \text { and } \quad-\tilde{g}_{3}^{*}\left(t_{1}, 1\right)=\rho_{12}^{2} t_{1}\left(t_{1}-2\right)+\rho_{23}^{2}
$$

in $t_{1}$ has the factor $\bar{\psi}_{2}^{*}(1)$, i.e.,

$$
\begin{aligned}
& R_{t_{1}}\left(\tilde{g}_{1}\left(t_{1}, 1\right),-\tilde{g}_{3}^{*}\left(t_{1}, 1\right)\right):=\left|\begin{array}{ccccc}
r^{2} & \rho_{12}^{2}+3 r^{2} & 2 \rho_{23}^{2}-4 \rho_{12}^{2} & \rho_{23}^{2}-4 r^{2} \\
& r^{2} & \rho_{12}^{2}+3 r^{2} & 2 \rho_{23}^{2}-4 \rho_{12}^{2} & \rho_{23}^{2}-4 r^{2} \\
\rho_{12}^{2} & -2 \rho_{12}^{2} & \rho_{23}^{2} & & \\
& \rho_{12}^{2} & -2 \rho_{12}^{2} & \rho_{23}^{2} & \\
& \rho_{12}^{2} & -2 \rho_{12}^{2} & \rho_{23}^{2}
\end{array}\right| \\
&=\left(\rho_{23}^{2}-\rho_{12}^{2}\right) \rho_{12}^{4} r^{2}\left(4 r^{2}-\rho_{12}^{2}\right) \bar{\psi}_{2}^{*}(1) .
\end{aligned}
$$

Remark. From (7.11), calculating (7.12) for $t_{2}=1$ directly, (or calculating the above determinant directly), $\bar{\psi}_{2}^{*}(1)$ is evaluated as

$$
\rho_{12}^{4} r^{2}\left(4 r^{2}-\rho_{12}^{2}\right) \bar{\psi}_{2}^{*}(1)=\left(8 \rho_{12}^{2}+\rho_{23}^{2}\right)^{2} r^{4}+4 \rho_{12}^{2}\left(\rho_{23}^{2}-\rho_{12}^{2}\right)\left\{\left(4 \rho_{12}^{2}-\rho_{23}^{2}\right) r^{2}+\rho_{12}^{2} \rho_{23}^{2}\right\} .
$$

Let $t_{1}=\eta_{1}, \eta_{2}$ be the roots of the equation $-\tilde{g}_{3}^{*}\left(t_{1}, 1\right)=0$. Then by definition

$$
R_{t_{1}}\left(\tilde{g}_{1}\left(t_{1}, 1\right),-\tilde{g}_{3}^{*}\left(t_{1}, 1\right)\right)=r^{4} \rho_{12}^{6} \prod_{j=1}^{3} \prod_{k=1}^{2}\left(\zeta_{j}-\eta_{k}\right)=r^{4} \prod_{j=1}^{3}\left(c_{1}\left(\zeta_{j}\right)+2 c_{2}\left(\zeta_{j}\right)\right) .
$$

On the other hand, from (7.4), (7.8) and (7.10) of Lemma 7.1 we have

$$
\begin{aligned}
\bar{\psi}_{2}^{*}(1) & =\prod_{j=4}^{7}\left(1-\frac{V^{*}\left(\zeta_{j}\right)}{U^{*}\left(\zeta_{j}\right)}\right)=\prod_{j=4}^{7} \frac{U^{*}\left(\zeta_{j}\right)-V^{*}\left(\zeta_{j}\right)}{U^{*}\left(\zeta_{j}\right)}=\prod_{j=4}^{7} \frac{c_{0}^{*}\left(\zeta_{j}\right)+c_{1}^{*}\left(\zeta_{j}\right)}{\rho_{12}^{2} \zeta_{j}-\rho_{23}^{2}} \\
& =\frac{1}{\rho_{12}^{8}} \prod_{j=4}^{7} \frac{c_{1}\left(\zeta_{j}\right)+2 c_{2}\left(\zeta_{j}\right)}{\zeta_{j}-\rho_{23}^{2} / \rho_{12}^{2}}=\frac{4 r^{2}-\rho_{12}^{2}}{r^{2} \rho_{23}^{4}\left(\rho_{23}^{2}-\rho_{12}^{2}\right)^{2}} \prod_{j=4}^{7}\left(c_{1}\left(\zeta_{j}\right)+2 c_{2}\left(\zeta_{j}\right)\right) .
\end{aligned}
$$

(7.23), (7.25) and (7.26) imply the following identity

$$
\mathcal{N}\left(c_{1}+2 c_{2}\right):=\prod_{j=1}^{7}\left(c_{1}\left(\zeta_{j}\right)+2 c_{2}\left(\zeta_{j}\right)\right)=\rho_{23}^{4} \rho_{12}^{4}\left(\rho_{23}^{2}-\rho_{12}^{2}\right)^{3} \bar{\psi}_{2}^{*}(1)^{2} .
$$

\subsection{Discriminants}

In this subsection we define the discriminants, and show their explicit forms. 
Definition 7.5. We denote by $\operatorname{Discr}_{j}$ the discriminant of $\bar{\psi}_{j}(j=1,2)$ and by $\operatorname{Discr}_{3}$ the product of the values $\left(t_{2}-1\right)^{2}$ at $t_{2}=\zeta_{j}^{*}(4 \leq j \leq 7)$ as follows.

$$
\begin{aligned}
& \operatorname{Discr}_{1}=-\prod_{j=1}^{3} \bar{\psi}_{1}^{\prime}\left(\zeta_{j}\right)=\prod_{1 \leq j<k \leq 3}\left(\zeta_{j}-\zeta_{k}\right)^{2}, \\
& \operatorname{Discr}_{2}=\prod_{j=4}^{7} \bar{\psi}_{2}^{\prime}\left(\zeta_{j}\right)=\prod_{4 \leq j<k \leq 7}\left(\zeta_{j}-\zeta_{k}\right)^{2}, \\
& \operatorname{Discr}_{3}=\left\{\bar{\psi}_{2}^{*}(1)\right\}^{2}=\prod_{j=4}^{7}\left(\zeta_{j}^{*}-1\right)^{2} .
\end{aligned}
$$

Lemma 7.6. Discr 1 is explicitly calculated as

$$
\operatorname{Discr}_{1}=r^{-8}\left(4 \rho_{12}^{2}-\rho_{23}^{2}\right) A=-\frac{B(0123)}{r^{8} \rho_{23}^{2}} A,
$$

where $A$ is given as the following polynomial in $r^{2}, \rho_{12}^{2}$ and $\rho_{23}^{2}$ :

$$
A=324 r^{6}+9\left(16 \rho_{12}^{2}-13 \rho_{23}^{2}\right) r^{4}+4\left(\rho_{12}^{2}-\rho_{23}^{2}\right)\left\{\left(23 \rho_{12}^{2}-8 \rho_{23}^{2}\right) r^{2}+\rho_{12}^{4}\right\} .
$$

Proof. From (7.20) we have $r^{2} \bar{\psi}_{1}\left(t_{1}\right)=\tilde{g}_{1}\left(t_{1}, 1\right)=\tilde{a}_{0} t_{1}^{3}+\tilde{a}_{1} t_{1}^{2}+\tilde{a}_{2} t_{1}+\tilde{a}_{3}$, where

$$
\tilde{a}_{0}=r^{2}, \quad \tilde{a}_{1}=\rho_{12}^{2}+3 r^{2}, \quad \tilde{a}_{2}=2\left(\rho_{23}^{2}-2 \rho_{12}^{2}\right), \quad \tilde{a}_{3}=\rho_{23}^{2}-4 r^{2} .
$$

The resultant of $\tilde{g}_{1}\left(t_{1}, 1\right)$ and its derivative gives the discriminant of $\bar{\psi}_{1}\left(t_{1}\right)$, i.e.,

$$
R\left(\tilde{g}_{1}\left(t_{1}, 1\right), \frac{d}{d t_{1}} \tilde{g}_{1}\left(t_{1}, 1\right)\right)=\left|\begin{array}{ccccc}
\tilde{a}_{0} & \tilde{a}_{1} & \tilde{a}_{2} & \tilde{a}_{3} & \\
& \tilde{a}_{0} & \tilde{a}_{1} & \tilde{a}_{2} & \tilde{a}_{3} \\
3 \tilde{a}_{0} & 2 \tilde{a}_{1} & \tilde{a}_{2} & & \\
& 3 \tilde{a}_{0} & 2 \tilde{a}_{1} & \tilde{a}_{2} & \\
& & 3 \tilde{a}_{0} & 2 \tilde{a}_{1} & \tilde{a}_{2}
\end{array}\right|=-\tilde{a}_{0}^{5} \prod_{1 \leq j<k \leq 3}\left(\zeta_{j}-\zeta_{k}\right)^{2}
$$

From the direct calculation of the above determinant, we obtain

$$
\begin{aligned}
& R\left(\tilde{g}_{1}\left(t_{1}, 1\right), \frac{d}{d t_{1}} \tilde{g}_{1}\left(t_{1}, 1\right)\right) \\
& \quad=r^{2}\left(\rho_{23}^{2}-4 \rho_{12}^{2}\right)\left\{324 r^{6}+9\left(16 \rho_{12}^{2}-13 \rho_{23}^{2}\right) r^{4}+4\left(\rho_{12}^{2}-\rho_{23}^{2}\right)\left(\left(23 \rho_{12}^{2}-8 \rho_{23}^{2}\right) r^{2}+\rho_{12}^{4}\right)\right\} .
\end{aligned}
$$

Since we have $B(0123)=-\rho_{23}^{2}\left(4 \rho_{12}^{2}-\rho_{23}^{2}\right)$ when $\rho_{13}=\rho_{12}$, this implies (7.28).

Lemma 7.7. Discr 2 is explicitly calculated as

$$
\operatorname{Discr}_{2}=\frac{\rho_{23}^{12}\left(\rho_{12}^{2}-\rho_{23}^{2}\right)^{2}\left(\rho_{12}^{2}+12 r^{2}\right)^{2}}{\rho_{12}^{16}\left(\rho_{12}^{2}-4 r^{2}\right)^{6}} C=\frac{\rho_{23}^{12}\left(\rho_{12}^{2}-\rho_{23}^{2}\right)^{2}}{\rho_{12}^{4}\{B(0 \star 12)\}^{6}} B^{2} C,
$$

where $B$ and $C$ are given as the following polynomials in $r^{2}, \rho_{12}^{2}$ and $\rho_{23}^{2}$ :

$$
\begin{aligned}
& B=12 r^{2}+\rho_{12}^{2}, \\
& C=\left(8 \rho_{12}^{2}+\rho_{23}^{2}\right)^{2} r^{4}+4 \rho_{12}^{2}\left(\rho_{23}^{2}-\rho_{12}^{2}\right)\left\{\left(4 \rho_{12}^{2}-\rho_{23}^{2}\right) r^{2}+\rho_{12}^{2} \rho_{23}^{2}\right\} .
\end{aligned}
$$


Proof. From the expression (7.5) of $U\left(t_{1}\right)=u_{0} \bar{\psi}_{2}\left(t_{1}\right)$, the resultant of $U\left(t_{1}\right)$ and $U^{\prime}\left(t_{1}\right)$ gives the discriminant of $\bar{\psi}_{2}\left(t_{1}\right)$, i.e.,

$$
R\left(U\left(t_{1}\right), U^{\prime}\left(t_{1}\right)\right)=\left|\begin{array}{ccccccc}
u_{0} & u_{1} & u_{2} & u_{3} & u_{4} & & \\
& u_{0} & u_{1} & u_{2} & u_{3} & u_{4} & \\
& & u_{0} & u_{1} & u_{2} & u_{3} & u_{4} \\
4 u_{0} & 3 u_{1} & 2 u_{2} & u_{3} & & \\
& 4 u_{0} & 3 u_{1} & 2 u_{2} & u_{3} & \\
& & 4 u_{0} & 3 u_{1} & 2 u_{2} & u_{3} & \\
& & & 4 u_{0} & 3 u_{1} & 2 u_{2} & u_{3}
\end{array}\right|=u_{0}^{7} \prod_{4 \leq j<k \leq 7}\left(\zeta_{j}-\zeta_{k}\right)^{2} .
$$

Calculating the above determinant directly, we consequently obtain

$$
\begin{aligned}
R\left(U\left(t_{1}\right), U^{\prime}\left(t_{1}\right)\right)=- & \rho_{12}^{12} \rho_{23}^{12}\left(\rho_{12}^{2}-4 r^{2}\right)\left(12 r^{2}+\rho_{12}^{2}\right)^{2}\left(\rho_{12}^{2}-\rho_{23}^{2}\right)^{2} \\
& \times\left\{\left(8 \rho_{12}^{2}+\rho_{23}^{2}\right)^{2} r^{4}+4 \rho_{12}^{2}\left(\rho_{23}^{2}-\rho_{12}^{2}\right)\left(\left(4 \rho_{12}^{2}-\rho_{23}^{2}\right) r^{2}+\rho_{12}^{2} \rho_{23}^{2}\right)\right\},
\end{aligned}
$$

which implies (7.30).

Remark. From (7.24) in Remark of Lemma 7.4, we have already known that $C$ is written as

$$
C=r^{2} \rho_{12}^{4}\left(4 r^{2}-\rho_{12}^{2}\right) \bar{\psi}_{2}^{*}(1)=-r^{2} \rho_{12}^{2} B(0 \star 12) \bar{\psi}_{2}^{*}(1) .
$$

Lemma 7.8. Suppose that $\rho_{12}^{2}-\rho_{23}^{2} \neq 0$. For the critical points $\mathbf{c}_{j}(4 \leq j \leq 7)$ under the assumption (7.13), $C=0$ if and only if $\mathbf{c}_{4}=\mathbf{c}_{6}$ or $\mathbf{c}_{5}=\mathbf{c}_{7}$. Then one of the pairs of them lie on the midline $L_{13}(x)-L_{12}(x)=0$ of $\triangle O_{1} O_{2} O_{3}$.

Proof. First we suppose $C=0$. From (7.32), we have $C=-r^{2} \rho_{12}^{2} B(0 \star 12) \prod_{j=4}^{7}\left(1-\zeta_{j}^{*}\right)=0$, so that there exists $j \in\{4,5,6,7\}$ such that $\zeta_{j}^{*}=1$. Then under the assumption (7.13) we have $\zeta_{4}^{*}=\zeta_{6}^{*}=1$ or $\zeta_{5}^{*}=\zeta_{7}^{*}=1$. Using Corollary 7.3 we have $\zeta_{4}=\zeta_{6}=-\hat{u}_{1}(1) / \hat{u}_{0}(1)$ or $\zeta_{5}=\zeta_{7}=-\hat{u}_{1}(1) / \hat{u}_{0}(1)$, where $\hat{u}_{1}(1)$ and $\hat{u}_{0}(1)$ are given by (7.18) and (7.19), respectively, so that we obtain $\mathbf{c}_{4}=\mathbf{c}_{6}$ or $\mathbf{c}_{5}=\mathbf{c}_{7}$. Here we needed to confirm that $\hat{u}_{0}(1) \neq 0$ for the precondition of Corollary 7.3. Assume that $\hat{u}_{0}(1)=0$. From the explicit form (7.19) of $\hat{u}_{0}(1)$, under $\rho_{12}^{2}-\rho_{23}^{2} \neq 0$ we have $4 r^{2}\left(4 \rho_{12}^{2}-\rho_{23}^{2}\right)-\rho_{12}^{2}\left(4 \rho_{12}^{2}-3 \rho_{23}^{2}\right)=0$. Since we see $4 \rho_{12}^{2}-\rho_{23}^{2}=-\rho_{23}^{-2} B(0123) \neq 0$, we have $r^{2}=\rho_{12}^{2}\left(4 \rho_{12}^{2}-3 \rho_{23}^{2}\right) / 4\left(4 \rho_{12}^{2}-\rho_{23}^{2}\right)$. Applying this to the explicit form (7.31) of $C$, we can calculate $C$ directly as $C=\rho_{12}^{4} \rho_{23}^{4}\left(4 \rho_{12}^{2}+5 \rho_{23}^{2}\right)^{2} / 16\left(4 \rho_{12}^{2}-\rho_{23}^{2}\right)^{2}$, which does not vanish. This contradicts $C=0$, and $\hat{u}_{0}(1) \neq 0$ is now confirmed.

Conversely, we next suppose $\mathbf{c}_{4}=\mathbf{c}_{6}$, namely $\left(\zeta_{4}, \zeta_{4}^{*}\right)=\left(\zeta_{6}, \zeta_{6}^{*}\right)$. From (7.13) we have $\zeta_{4}^{*}=\zeta_{6}^{*}=1 / \zeta_{4}^{*}$, so that $\zeta_{4}^{* 2}=1$, i.e., $\zeta_{4}^{*}= \pm 1$. Here we claim $\zeta_{4}^{*}=1$ by contradiction. Assume that $\zeta_{4}^{*}=-1$. Since $\zeta_{4}^{*}$ is a root of the polynomial $\bar{\psi}_{2}^{*}\left(t_{2}\right)$, we have $\bar{\psi}_{2}^{*}(-1)=\bar{\psi}_{2}^{*}\left(\zeta_{4}^{*}\right)=0$. The identity (7.11) for $t_{2}=-1$ implies $R_{t_{1}}\left(\tilde{g}_{2}^{*}\left(t_{1},-1\right),-\tilde{g}_{3}^{*}\left(t_{1},-1\right)\right)=r^{2}\left(4 r^{2}-\rho_{12}^{2}\right) \rho_{12}^{4} \bar{\psi}_{2}^{*}(-1)=0$, which means the polynomials $\tilde{g}_{2}^{*}\left(t_{1},-1\right)=\left(r^{2}-\rho_{12}^{2}\right) t_{1}^{2}-\rho_{23}^{2}$ and $\tilde{g}_{3}^{*}\left(t_{1},-1\right)=\rho_{12}^{2} t_{1}^{2}-\rho_{23}^{2}=\left(\rho_{12} t_{1}-\rho_{23}\right)\left(\rho_{12} t_{1}+\rho_{23}\right)$ have common zero points. Hence we have $0=\tilde{g}_{2}^{*}\left( \pm \rho_{23} / \rho_{12},-1\right)=\rho_{23}^{2}\left(r^{2}-2 \rho_{12}^{2}\right) / \rho_{12}^{2}$, so that $r^{2}-2 \rho_{12}^{2}=0$. From Lemma 7.7, for $r^{2}=2 \rho_{12}^{2}$ the discriminant Discr 2 is evaluated as Discr $2=$ $2^{2} 5^{4} \rho_{23}^{12}\left(\rho_{12}^{2}-\rho_{23}^{2}\right)^{2}\left(56 \rho_{12}^{2}+25 \rho_{23}^{2}\right) / 7^{6} \rho_{12}^{18}$. Under $\rho_{12}^{2}-\rho_{23}^{2} \neq 0$, we have Discr $2 \neq 0$, which means $\bar{\psi}_{2}\left(t_{1}\right)=\prod_{j=4}^{7}\left(t_{1}-\zeta_{j}\right)$ has no double roots. This contradicts $\zeta_{4}=\zeta_{6}$. Thus we obtain $\zeta_{4}^{*}=\zeta_{6}^{*}=1$, so that $C=-r^{2} \rho_{12}^{2} B(0 \star 12) \prod_{j=4}^{7}\left(1-\zeta_{j}^{*}\right)=0$.

Consequently, one of the pairs of the critical points lie on the line defined by $t_{2}=1$. 
Remark. When the situation $\mathbf{c}_{4}=\mathbf{c}_{6}$ occurred as Lemma 7.8, these two actually coincide with real one in $\left\{\mathbf{c}_{1}, \mathbf{c}_{2}, \mathbf{c}_{3}\right\}$ simultaneously. We can confirm this in the following way. From Corollary 7.3 the coordinates of $\mathbf{c}_{4}=\mathbf{c}_{6}$ are given as $\left(t_{1}, t_{2}\right)=\left(-\hat{u}_{1}(1) / \hat{u}_{0}(1), 1\right)$, where

$$
-\frac{\hat{u}_{1}(1)}{\hat{u}_{0}(1)}=-\frac{\rho_{23}^{2}\left\{r^{2}\left(\rho_{23}^{2}-16 \rho_{12}^{2}\right)+2 \rho_{12}^{2}\left(2 \rho_{12}^{2}-\rho_{23}^{2}\right)\right\}}{2 \rho_{12}^{2}\left\{4 r^{2}\left(4 \rho_{12}^{2}-\rho_{23}^{2}\right)-\rho_{12}^{2}\left(4 \rho_{12}^{2}-3 \rho_{23}^{2}\right)\right\}} .
$$

For our purpose it suffices to see that $t_{1}=-\hat{u}_{1}(1) / \hat{u}_{0}(1)$ indeed satisfy the equation $\tilde{g}_{1}\left(t_{1}, 1\right)=0$ given in (7.20), and in fact, $\tilde{g}_{1}\left(-\hat{u}_{1}(1) / \hat{u}_{0}(1), 1\right)$ is divisible by $C$ as

$$
\tilde{g}_{1}\left(-\hat{u}_{1}(1) / \hat{u}_{0}(1), 1\right)=C D\left(2 \rho_{12}^{2}\right)^{-3}\left\{4 r^{2}\left(4 \rho_{12}^{2}-\rho_{23}^{2}\right)-\rho_{12}^{2}\left(4 \rho_{12}^{2}-3 \rho_{23}^{2}\right)\right\}^{-3},
$$

where $C$ is given in (7.31) and $D$ has relatively longer expression as

$$
\begin{aligned}
D= & r^{4}\left(8 \rho_{12}^{2}-\rho_{23}^{2}\right)^{2}\left(32 \rho_{12}^{4}-24 \rho_{12}^{2} \rho_{23}^{2}+\rho_{23}^{4}\right) \\
& -2 r^{2} \rho_{12}^{2}\left(512 \rho_{12}^{8}-768 \rho_{12}^{6} \rho_{23}^{2}+348 \rho_{12}^{4} \rho_{23}^{4}-39 \rho_{12}^{2} \rho_{23}^{6}+\rho_{23}^{8}\right) \\
& +2\left(4 \rho_{12}^{2}-3 \rho_{23}^{2}\right)\left(16 \rho_{12}^{4}-20 \rho_{12}^{2} \rho_{23}^{2}+7 \rho_{23}^{4}\right) .
\end{aligned}
$$

As we saw in Lemma $7.8, C=0$ if and only if $\mathbf{c}_{4}=\mathbf{c}_{6}$, thus $\tilde{g}_{1}\left(-\hat{u}_{1}(1) / \hat{u}_{0}(1), 1\right)=0$ is confirmed.

Lemma 7.9. Discr $_{3}$ is explicitly calculated as

$$
\operatorname{Discr}_{3}=\frac{C^{2}}{r^{4} \rho_{12}^{8}\left(\rho_{12}^{2}-4 r^{2}\right)^{2}}=\frac{C^{2}}{r^{4} \rho_{12}^{4}\{B(0 \star 12)\}^{2}},
$$

where $C$ is the polynomial in $r^{2}, \rho_{12}^{2}$ and $\rho_{23}^{2}$ given by (7.31) in Lemma 7.7.

Proof. By definition we immediately have (7.33) from the expression (7.32) of $\bar{\psi}_{2}^{*}(1)$.

\subsection{Main Results for Isosceles Triangle Case}

Due to (7.3) by taking the limit $\rho_{13}^{2} \rightarrow \rho_{12}^{2}$ in Lemma 5.6 we have

Lemma 7.10. $R^{*}$ and $W^{*}$ are expressed as the following polynomials in $t_{1}$, respectively.

$$
\begin{aligned}
R^{*} & =-r^{2} \rho_{12}^{4}\left(\rho_{12}^{2}-4 r^{2}\right) t_{1} \bar{\psi}_{1}\left(t_{1}\right) \bar{\psi}_{2}\left(t_{1}\right), \\
\left(c_{1}+2 c_{2}\right) W^{*} & =t_{1}\left\{r^{2}\left(\rho_{12}^{2} t_{1}-\rho_{23}^{2}\right)^{2} \bar{\psi}_{1}\left(t_{1}\right)+\rho_{12}^{4}\left(\rho_{12}^{2}-4 r^{2}\right)\left(t_{1}-1\right) \bar{\psi}_{2}\left(t_{1}\right)\right\} .
\end{aligned}
$$

Proof . From Lemma 5.6 we have $R^{*}\left(t_{1}\right)=-r^{2} \rho_{12}^{4}\left(\rho_{12}^{2}-4 r^{2}\right) t_{1} \bar{\psi}\left(t_{1}\right)$, where the characteristic polynomial $\bar{\psi}\left(t_{1}\right)=\prod_{j=1}^{7}\left(t_{1}-\zeta_{j}\right)$ is equal to the product of two factors $\bar{\psi}_{1}\left(t_{1}\right)$ and $\bar{\psi}_{2}\left(t_{1}\right)$, i.e., $\bar{\psi}\left(t_{1}\right)=\bar{\psi}_{1}\left(t_{1}\right) \bar{\psi}_{2}\left(t_{1}\right)$. This implies (7.34). From (7.3) we obtain

$$
\begin{aligned}
\left(c_{1}+2 c_{2}\right) W^{*} & =c_{0}^{2} \frac{R^{*}}{U}-t_{1}\left(t_{1}-1\right) U=\left(\rho_{12}^{2} t_{1}-\rho_{23}^{2}\right)^{2} \frac{R^{*}\left(t_{1}\right)}{U\left(t_{1}\right)}-t_{1}\left(t_{1}-1\right) U\left(t_{1}\right) \quad(\text { using (7.1)) } \\
& =\left(\rho_{12}^{2} t_{1}-\rho_{23}^{2}\right)^{2} \frac{r^{2} t_{1} \bar{\psi}\left(t_{1}\right)}{\bar{\psi}_{2}\left(t_{1}\right)}+t_{1}\left(t_{1}-1\right) \rho_{12}^{4}\left(\rho_{12}^{2}-4 r^{2}\right) \bar{\psi}_{2}\left(t_{1}\right) \quad(\text { using (7.5)), }
\end{aligned}
$$

which coincides with (7.35). 
Lemma 7.11. We have

(1) $\left[\frac{R^{* \prime}}{W^{*}}\right]_{\zeta_{j}}=-r^{2}\left[\frac{c_{1}+2 c_{2}}{t_{1}-1} \bar{\psi}_{1}^{\prime}\left(t_{1}\right)\right]_{\zeta_{j}} \quad(1 \leq j \leq 3)$,

(2) $\left[\frac{R^{* \prime}}{W^{*}}\right]_{\zeta_{j}}=-\rho_{12}^{4}\left(\rho_{12}^{2}-4 r^{2}\right)\left[\frac{c_{1}+2 c_{2}}{c_{0}^{2}} \bar{\psi}_{2}^{\prime}\left(t_{1}\right)\right]_{\zeta_{j}} \quad(4 \leq j \leq 7)$.

Proof. For $1 \leq j \leq 3 \bar{\psi}_{1}\left(\zeta_{j}\right)=0$. Due to (7.34) and (7.35) we have

$$
\begin{aligned}
{\left[\left(c_{1}+2 c_{2}\right) W^{*}\right]_{\zeta_{j}} } & =\rho_{12}^{4}\left(\rho_{12}^{2}-4 r^{2}\right)\left[t_{1}\left(t_{1}-1\right) \bar{\psi}_{2}\left(t_{1}\right)\right]_{\zeta_{j}}, \\
{\left[R^{* \prime}\right]_{\zeta_{j}} } & =-r^{2} \rho_{12}^{4}\left(\rho_{12}^{2}-4 r^{2}\right)\left[t_{1} \bar{\psi}_{1}^{\prime}\left(t_{1}\right) \bar{\psi}_{2}\left(t_{1}\right)\right]_{\zeta_{j}},
\end{aligned}
$$

hence (1) follows. Likewise for $4 \leq j \leq 7 \bar{\psi}_{2}\left(\zeta_{j}\right)=0$, so that we have

$$
\begin{aligned}
{\left[\left(c_{1}+2 c_{2}\right) W^{*}\right]_{\zeta_{j}} } & =r^{2}\left[\left(\rho_{12}^{2} t_{1}-\rho_{23}^{2}\right)^{2} t_{1} \bar{\psi}_{1}\left(t_{1}\right)\right]_{\zeta_{j}}, \\
{\left[R^{* \prime}\right]_{\zeta_{j}} } & =-r^{2} \rho_{12}^{4}\left(\rho_{12}^{2}-4 r^{2}\right)\left[t_{1} \bar{\psi}_{2}^{\prime}\left(t_{1}\right) \bar{\psi}_{1}\left(t_{1}\right)\right]_{\zeta_{j}},
\end{aligned}
$$

hence (2) follows.

If $c_{1}+2 c_{2}=0$ then $t_{2}$ defined by (7.7) equals 1 . Furthermore

Lemma 7.12. Suppose that $\rho_{23}^{2} \neq \rho_{12}^{2}$ and $c_{1}+2 c_{2}=0$. Then, $\bar{\psi}_{1}\left(t_{1}\right)=0$ if and only if $\bar{\psi}_{2}\left(t_{1}\right)=0$.

Proof. Indeed since $\left(t_{1}-1\right)\left(\rho_{12}^{2} t_{1}-\rho_{23}^{2}\right) \neq 0$, (7.35) implies Lemma 7.12.

As a consequence of Lemma 7.11 one sees that if $c_{1}+2 c_{2}=0, \bar{\psi}_{1}\left(t_{1}\right)=0$, then $\bar{\psi}_{2}\left(t_{1}\right)=0$. Conversely if $c_{1}+2 c_{2}=0, \bar{\psi}_{2}\left(t_{1}\right)=0$, then $\bar{\psi}_{1}\left(t_{1}\right)=0$.

Proposition 7.13.

$$
\mathcal{N}\left(\frac{R^{* \prime}}{W^{*}}\right):=\prod_{j=1}^{7} \frac{R^{* \prime}\left(\zeta_{j}\right)}{W^{*}\left(\zeta_{j}\right)}=\frac{r^{4} \rho_{12}^{20}\left(\rho_{12}^{2}-4 r^{2}\right)^{6}}{3 \rho_{23}^{4}\left(\rho_{23}^{2}-\rho_{12}^{2}\right)^{2}} \bar{\psi}_{2}^{*}(1)^{2} \prod_{j=1}^{3} \bar{\psi}_{1}^{\prime}\left(\zeta_{j}\right) \prod_{j=4}^{7} \bar{\psi}_{2}^{\prime}\left(\zeta_{j}\right) .
$$

Proof. Indeed Lemma 7.11 means

$$
\prod_{j=1}^{7} \frac{R^{* \prime}\left(\zeta_{j}\right)}{W^{*}\left(\zeta_{j}\right)}=-r^{6}\left\{\rho_{12}^{4}\left(\rho_{12}^{2}-4 r^{2}\right)\right\}^{4} \mathcal{N}\left(c_{1}+2 c_{2}\right) \prod_{j=1}^{3} \frac{\bar{\psi}_{1}^{\prime}\left(\zeta_{j}\right)}{\zeta_{j}-1} \prod_{j=4}^{7} \frac{\bar{\psi}_{2}^{\prime}\left(\zeta_{j}\right)}{c_{0}^{2}\left(\zeta_{j}\right)}
$$

Proposition 7.13 follows from (7.21), (7.22) and (7.27).

The interpolating curve $t_{2}=\omega\left(t_{1}\right)$ is thus defined by

$$
t_{2}=\omega\left(t_{1}\right):=\left\{\begin{array}{cll}
1 & \text { if } & t_{1} \in\left\{\zeta_{1}, \zeta_{2}, \zeta_{3}\right\}, \\
\frac{\rho_{12}^{2} t_{1}\left(t_{1}-1\right)}{\rho_{12}^{2} t_{1}-\rho_{23}^{2}} & \text { if } & t_{1} \in\left\{\zeta_{4}, \zeta_{5}, \zeta_{6}, \zeta_{7}\right\} .
\end{array}\right.
$$

Under the condition $\rho_{12}^{2}=\rho_{13}^{2}$ (isosceles triangle case, so that $B(0 \star 12)=B(0 \star 13)$ ), we eventually obtain the same formulae of $\mathcal{N}\left(f_{j}\right)(1 \leq j \leq 3)$ as in Theorem 5.19 which was proved under $\rho_{12}^{2} \neq \rho_{13}^{2}$; that is 
Theorem 7.14.

$$
\begin{aligned}
& \mathcal{N}\left(f_{1}\right)=\prod_{j=1}^{7}\left[f_{1}\right]_{\mathbf{c}_{j}}=\frac{r^{2}}{2 \cdot 3^{4}} \frac{B(0 \star 12) B(0 \star 13) B(0 \star 123)}{\rho_{23}^{2}}, \\
& \mathcal{N}\left(f_{2}\right)=\prod_{j=1}^{7}\left[f_{2}\right]_{\mathbf{c}_{j}}=\frac{r^{2}}{2 \cdot 3^{4}} \frac{B(0 \star 23) B(0 \star 12) B(0 \star 123)}{\rho_{13}^{2}}, \\
& \mathcal{N}\left(f_{3}\right)=\prod_{j=1}^{7}\left[f_{3}\right]_{\mathbf{c}_{j}}=\frac{r^{2}}{2 \cdot 3^{4}} \frac{B(0 \star 23) B(0 \star 13) B(0 \star 123)}{\rho_{12}^{2}} .
\end{aligned}
$$

From (7.6) and Proposition 7.13 we finally obtain the following formula in view of the equality $B(0 \star 12)=\rho_{12}^{2}\left(\rho_{12}^{2}-4 r^{2}\right)$.

Theorem 7.15.

$$
\mathcal{N}(\operatorname{Hess}(F))=-\frac{r^{4} \rho_{12}^{8}\{B(0 \star 12)\}^{6}}{3 \rho_{23}^{4}\left(\rho_{23}^{2}-\rho_{12}^{2}\right)^{2}} \mathcal{N}\left(\frac{t_{1} t_{2}}{t_{\infty} f_{3}^{4}}\right) \operatorname{Discr}_{1} \operatorname{Discr}_{2} \operatorname{Discr}_{3},
$$

where $\mathcal{N}\left(t_{j}\right)(j=1,2), \mathcal{N}\left(t_{\infty}\right)$ and $\mathcal{N}\left(f_{3}\right)$ are given by Propositions 5.7, 5.11 and Theorem 7.14, respectively.

Remark. Writing the factor $\mathcal{N}\left(t_{1} t_{2} / t_{\infty} f_{3}{ }^{4}\right)$ in (7.36) explicitly, $\mathcal{N}(\operatorname{Hess}(F))$ is expressed as

$$
\mathcal{N}(\operatorname{Hess}(F))=\frac{2^{4} 3^{12} \rho_{12}^{12}}{\rho_{23}^{4}\left(\rho_{23}^{2}-\rho_{12}^{2}\right)^{2}} \frac{\{B(0 \star 12)\}^{2}}{B(0123)\{B(0 \star 23)\}^{3}\{B(0 \star 123)\}^{4}} \text { Discr }_{1} \text { Discr }_{2} \text { Discr }_{3} .
$$

One may conclude the following:

Corollary 7.16. Suppose that $\rho_{13}^{2}-\rho_{12}^{2}=0$ and $\rho_{23}^{2}-\rho_{12}^{2} \neq 0$. Two critical points in the set $\left\{\mathbf{c}_{j} \mid 1 \leq j \leq 7\right\}$ coincide with each other if and only if

$$
\mathcal{N}(\operatorname{Hess}(F))=0
$$

From Lemmas 7.6, 7.7 and 7.9, $\mathcal{N}(\operatorname{Hess}(F))$ is also written in the following form.

Corollary 7.17. Suppose that $\rho_{13}^{2}-\rho_{12}^{2}=0$ and $\rho_{23}^{2}-\rho_{12}^{2} \neq 0 . \mathcal{N}(\operatorname{Hess}(F))$ is expressed as

$$
\mathcal{N}(\operatorname{Hess}(F))=-\frac{2^{4} 3^{12} \rho_{12}^{4} \rho_{23}^{6}}{r^{12}} \frac{A B^{2} C^{3}}{\{B(0 \star 12)\}^{6}\{B(0 \star 23)\}^{3}\{B(0 \star 123)\}^{4}},
$$

where $A$ and $B, C$ are the polynomials in $r^{2}, \rho_{12}^{2}, \rho_{23}^{2}$ given in (7.29) and (7.31), respectively. One can say more precisely that

(1) A = 0 if and only if two points in $\left\{\mathbf{c}_{j} \mid 1 \leq j \leq 3\right\}$ coincide with each other,

(2) $B=0$ if and only if two points in $\left\{\mathbf{c}_{j} \mid 4 \leq j \leq 7\right\}$ such that $t_{2} \neq 1$ coincide with each other,

(3) $C=0$ if and only if two points in $\left\{\mathbf{c}_{j} \mid 4 \leq j \leq 7\right\}$ coincide with each other, so that $t_{2}=1$. 
Remark 1. In fact, $B=0$ occurs only when $r^{2}=-\rho_{12}^{2} / 12<0$. Moreover, $B=C=0$ occurs only once when $25 \rho_{23}^{2}-16 \rho_{12}^{2}=0$. Then all the points in $\left\{\mathbf{c}_{j} \mid 4 \leq j \leq 7\right\}$ coincide with one of those in $\left\{\mathbf{c}_{j} \mid 1 \leq j \leq 3\right\}$ defined by $\left(t_{1}, t_{2}\right)=(2 / 5,1)$, so that five of the critical points overlap quintuply on the midline of $\triangle O_{1} O_{2} O_{3}$ defined by $t_{2}=1$, while the other two are not real.

Remark 2. Lemma 7.8 gives a detailed explanation of Corollary 7.17 (3).

Remark 3. There exists a unique positive $r=r_{c}$ satisfying the following properties:

(1) If $\rho_{12}^{2}=\rho_{13}^{2}>\rho_{23}^{2}$, then $A>0$ and $C=0$.

(2) If $\rho_{23}^{2}>\rho_{12}^{2}=\rho_{13}^{2}$, then $A=0$ and $C>0$.

(3) If $\rho_{12}^{2}=\rho_{13}^{2}=\rho_{23}^{2}$, i.e., if $\triangle O_{1} O_{2} O_{3}$ is a regular triangle, then $r_{c}=0$ such that $A=C=0$.

In each case for $r>r_{c}$ the conditions $A>0$ and $C>0$ are satisfied. Therefore, under the conditions $\rho_{13}^{2}=\rho_{12}^{2}$ and $\rho_{23}^{2} \neq \rho_{12}^{2}$ it is satisfied for $r>r_{c}$ that $\operatorname{Discr}_{1}>0$, Discr $_{2}>0$ and $\operatorname{Discr}_{3}>0$, which means that all the 7 critical points are real and different from each other.

Remark 4. From the similarity of triangles, without loss of generality we may fix the hight of the isosceles triangles to be $\sqrt{3}$. Using the length $L=\rho_{23} / 2$ the Pythagorean theorem says $\rho_{12}^{2}=L^{2}+3$. In this situation, using variables

$$
X=r^{2} \quad \text { and } \quad Y=L^{2} \geq 0,
$$

the conditions (1) $\rho_{12}^{2}=\rho_{13}^{2}>\rho_{23}^{2}$, (2) $\rho_{23}^{2}>\rho_{12}^{2}=\rho_{13}^{2}$ and (3) $\rho_{12}^{2}=\rho_{13}^{2}=\rho_{23}^{2}$ in Remark 3 are equivalent to (1) $0 \leq Y<1$, (2) $Y>1$ and (3) $Y=1$, respectively, and the discriminants $A, B, C$ are written as the polynomials in $X, Y$

$$
\begin{aligned}
& A=A(X, Y)=12\left[27 X^{3}-9(3 Y-4) X^{2}+(Y-1)\left\{3(3 Y-23) X-(Y+3)^{2}\right\}\right], \\
& B=B(X, Y)=12 X+Y+3, \\
& C=C(X, Y)=48\left[3(Y+2)^{2} X^{2}+(Y-1)(Y+3)\{3 X+Y(Y+3)\}\right],
\end{aligned}
$$

which satisfy $\operatorname{Discr}_{1}=12 A(X, Y) / X^{4}$ and

$$
\operatorname{Discr}_{2}=\left\{\frac{(4 Y)^{3} 3(Y-1) B(X, Y)}{(Y+3)\{B(0 \star 12)\}^{3}}\right\}^{2} C(X, Y), \quad \operatorname{Discr}_{3}=\left\{\frac{C(X, Y)}{X(Y+3) B(0 \star 12)}\right\}^{2} .
$$

Figure 3 below shows the graphs of $A(X, Y)=0, B(X, Y)=0$ and $C(X, Y)=0$ in the $X-Y$ plane with those of $B(0 \star 12)=(Y+3)(Y-4 X+3)=0, B(0 \star 23)=16 Y(Y-X)=0$ and $B(0 \star 123)=8 Y\left\{12 X-(Y+3)^{2}\right\}=0$. The 7 critical points corresponding to $(X, Y)$ in the shaded area, where $A(X, Y)>0$ and $C(X, Y)>0$, are all real and different from each other. Thus we can clearly understand the claims explained in Remark 3 when we see the shaded area in $X \geq 0$. Incidentally, the point $(X, Y)=(3,3)$ satistying $B(0 \star 123)=$ Discr $_{1}=0$ corresponds to the isosceles right triangle $\triangle O_{1} O_{2} O_{3}$ with the circles of radius $\sqrt{3}$. It is remarkable that, in a neighborhood of the point $(3,3)$ if $B(0 \star 123)>0$, then Discr $1>0$, while there exist infinitely many points such that $B(0 \star 123)<0$ and Discr $_{1}<0$. It would be also an interesting problem to investigate in detail about the critical points for $X=r^{2}<0$ (in the case of imaginary circles). Even in the part $X<0$ we still have the shaded areas of peculiar shape (Figure 4), and we can find that the line $B(X, Y)=0$ appears only in $X<0$. 


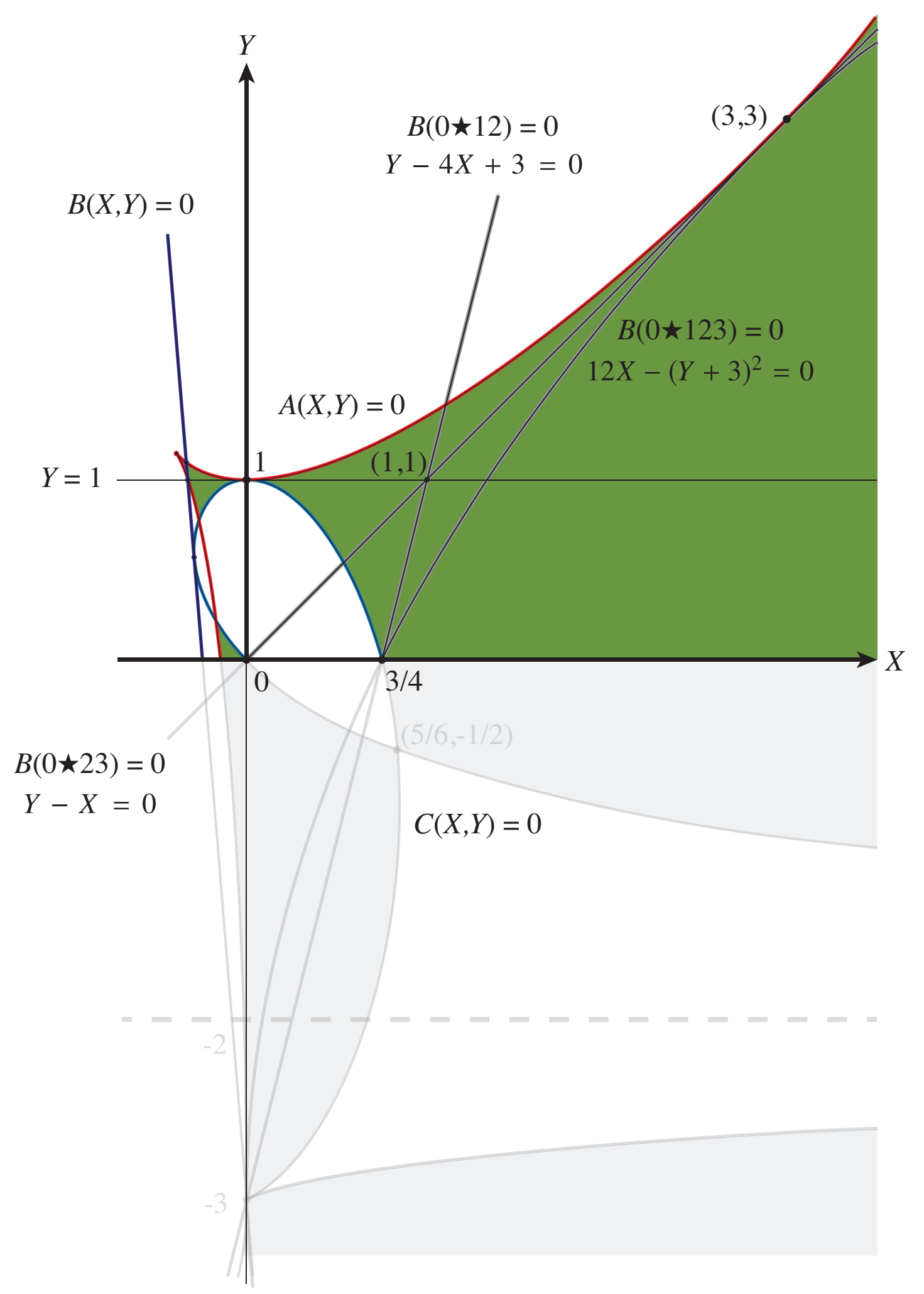

Figure 3: Graphs of the discriminants 


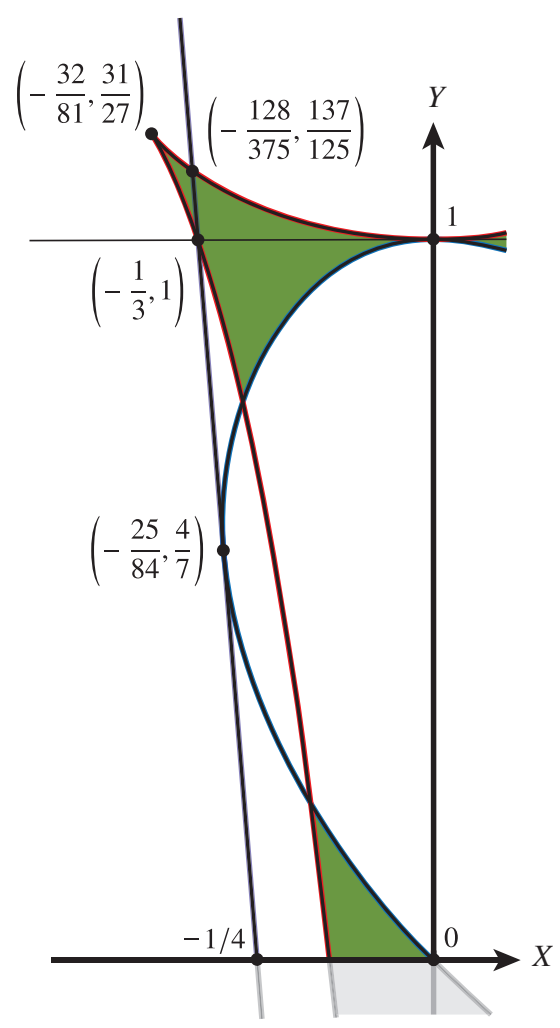

Figure 4: Quadrant of $X=r^{2}<0$

\section{References}

[1] S. S. Abhyanker, Ramification Theoretic Methods in Algebraic Geometry, Princeton UP, 1959.

[2] S. S. Abhyanker, On the semi-group of a meromorphic curve, Part I, in Proceedings of the International Symposium on Algebraic Geometry Kyoto (1977), 240 - 414.

[3] K. Aomoto and M. Kita, Theory of Hypergeometric Functions, Springer 2011.

[4] K. Aomoto and Y. Machida, Double filtration of twisted logarithmic complex and Gauss-Manin connection, J. Math. Soc. Japan 67 (2015), 609-636.

[5] K. Aomoto and Y. Machida, Some problems of hypergeometric integrals associated with hypersphere arrangement, Proc. Japan Acad. Ser. A Math. Sci. 91 (2015), 77-81.

[6] K. Aomoto and Y. Machida, Hypergeometric integrals associated with hypersphere arrangements and Cayley-Menger determinants, Hokkaido Math. J. 49 (2020), 1-85. [math. DG/1709.09329].

[7] K. Aomoto and Y. Machida, Generalization of Schläfli formula to the volume of a spherically faced simplex, J. Math. Soc. Japan 72 (2020), 213-240 [doi . 10. 2969/jmsj/80238023].

[8] F. R. Gantmacher, The theory of matrices Vol. 2, translated by K. A. Hirsch, Chelsea Publishing Co., New York 1959. 
[9] I. M. Gelfand and G. E. Shilov, Generalized functions Vol.1, Properties and operations, translated from the Russian by Eugene Saletan. Academic Press, New York-London, 1964.

[10] W. Gröbner, Moderne Algebraische Geometrie, Springer Verlag, 1949.

[11] H. Minkowski, Diophantische Approximationen, Chelsea, 1957.

[12] P. Orlik and H. Terao, Arrangements and Hypergeometric Integrals, MSJ Memoirs 9 (2001).

[13] T. Takagi, Lecture on Algebra (in Japanese), Kyouritu,1948.

[14] T. Takagi, Theory of Algebraic Numbers (in Japanese), 2nd Edition, Iwanami, 1971.

[15] M. Yoshida, Hypergeometric functions, my love. Modular interpretations of configuration spaces, Aspects of Mathematics E32, Friedr. Vieweg \& Sohn, Braunschweig, 1997. 